\title{
High-Resolution X-ray Emission and X-ray Absorption Spectroscopy
}

\author{
Frank de Groot \\ Department of Inorganic Chemistry and Catalysis, Utrecht University, Sorbonnelaan 16, 3584 CA Utrecht, Netherlands
}

Received July 31, 2000

\section{Contents}

I. Introduction

A. X-ray Absorption

II. 1s Edge X-ray Absorption

A. Metal 1s X-ray Absorption Spectra

1. Metal 1s Edge Shifts

B. The Preedge Region

C. Ligand 1s X-ray Absorption

III. $2 p$ Edge X-ray Absorption

A. Atomic Multiplet Theory

1. The Atomic Hamiltonian

2. Term Symbols

3. The Matrix Elements

4. Atomic Multiplet Ground States of $3 d^{N}$ Systems

5. X-ray Absorption Spectra Described with Atomic Multiplets

B. Ligand-Field Multiplet Theory

C. Charge-Transfer Multiplet Theory

IV. 1s X-ray Emission

A. 1 s3p X-ray Emission in the Ligand-Field Multiplet Model

B. 1 s3p X-ray Emission in the Charge-Transfer Multiplet Model

1. K Capture 1 s3p X-ray Emission

2. Temperature- and Pressure-Dependent 1 s3p X-ray Emission

C. 1s2p X-ray Emission

D. 1s2p Resonant X-ray Emission

E. 1s Valence Band $X$-ray Emission

V. $2 p$ X-ray Emission

A. 2p3s Resonant X-ray Emission

B. 2p3d Resonant $X$-ray Emission

1. Charge-Transfer Transitions and dd (ff) Transitions

2. Coster-Kronig Transitions

3. Spin-Flip Transitions

VI. X-ray Magnetic Circular Dichroism

A. MCD in X-ray Absorption

1. MLD in X-ray Absorption

B. MCD in X-ray Emission

1. MLD in X-ray Emission

E. Spin-Polarized 1s2p and 1s3p X-ray Emission

VII. Selective X-ray Absorption

A. Lifetime Broadening Removed X-ray Absorption

B. Valence-, Spin-, and Site-Selective X-ray Absorption
1779

1779

1780

1781

1781

1781

1782

1784

1784

1785

1785

1786

1787

1788

1789

1790

1792

1792

1793

1794

1794

1794

1795

1796

1797

1798

1799

1799

1800

1800

1801

1801

1802

1802

1803

1803

1804

1805

1805
1. Valence-Selective X-ray Absorption

2. Spin-Selective $X$-ray Absorption

3. Site-Selective X-ray Absorption

VIII. Conclusions and Outlook

IX. Acknowledgment

$X$. References

\section{Introduction}

In this review, high-resolution X-ray emission and X-ray absorption spectroscopy will be discussed. The focus is on the $3 d$ transition-metal systems. To understand high-resolution X-ray emission and resonant X-ray emission, it is first necessary to spend some time discussing the X-ray absorption process. Section II discusses Is X-ray absorption, i.e., the K edges, and section III deals with $2 p$ X-ray absorption, the $L$ edges. X-ray emission is discussed in, respectively, section IV on $1 s 2 p$ and $2 s 3 p$ X-ray emission and section $V$ on $2 p 3 s$ and $2 p 3 d$ X-ray emission. Section VI focuses on magnetic dichroism effects, and in section VII selective X-ray absorption experiments are discussed.

To limit the scope of this review paper, many related topics (for example, EELS, XPS, and resonant photoemission, phonon-oriented inelastic X-ray scattering, and X-ray microscopy) will not be discussed. In addition, many aspects of X-ray absorption, such as reflection experiments, diffraction absorption fine structure, and related experiments, will remain untouched. EXAFS will be discussed very briefly, and its X-ray emission analogue EXEFS ${ }^{69,71}$ will not be discussed.

\section{A. X-ray Absorption}

X-ray absorption is a synchrotron-based characterization technique that can be divided into nearedge spectroscopy (XANES) and extended X-ray absorption fine structure (EXAFS). In recent years a number of books, book chapters, and review papers have been published on X-ray absorption analysis. NEXAFS spectroscopy by Stöhr deals in detail with the analysis of soft X-ray 1s XANES spectra. ${ }^{101}$ A recent review on soft X-ray XANES has been written by Chen. ${ }^{24} \mathrm{H}$ ard X-ray XANES and EXAFS has been discussed in the book by Koningsberger and Prins. ${ }^{72}$ Rehr and Albers recently wrote a review on the theory of hard X-ray XANES and EXAFS. ${ }^{92}$ In the Encyclopaedia of Analytical Chemistry, a recent chapter on X-ray absorption has been written by Kawai. ${ }^{70}$ 


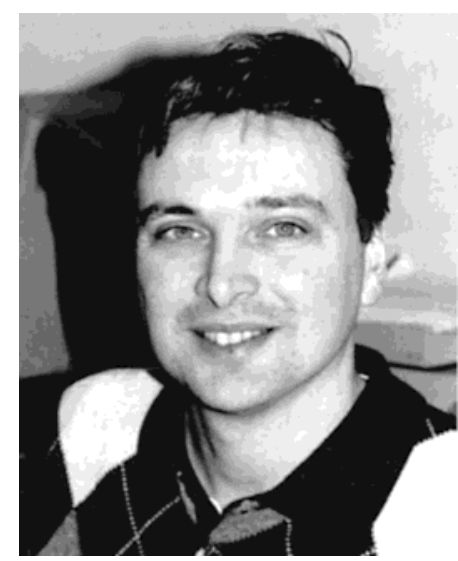

Frank M. F. de Groot grew up in Nuenen, The Netherlands, received his Ph.D. degree from the University of Nijmegen (with J. C. Fuggle), and was a postdoctoral fellow at the LURE Synchrotron in Orsay, France. He was a Royal Netherlands Academy of Arts (KNAW) fellow at the University of Groningen. He then moved to Utrecht University, where he is now Lecturer in the Department of Inorganic Chemistry and Catalysis. His research is in the fields of inorganic chemistry and solid-state chemistry and physics with emphasis on the basic understanding and application of core-level spectroscopies to study the electronic and magnetic structure of condensed matter, in particular the bulk and surfaces of transitionmetal oxides, magnetic nanoparticles, heterogeneous catalysts, and bioinorganic materials.

In the X-ray absorption process, a core electron is excited to an empty state and, as such, X-ray absorption probes the unoccupied part of the electronic structure of the system. ${ }^{52}$ In the EXAFS region, the excited electron has significant kinetic energy and EXAFS analysis yields for many systems an accurate determination of the local geometry. The XANES structure can also be simulated with the multiple scattering formalism. Alternatively, one can use electronic structure models such as density-functional theory to calculate the unoccupied density of states (DOS). ${ }^{101}$ An important aspect of the usefulness of $\mathrm{X}$-ray absorption is that one obtains el ement-specific information. This also implies that if two or more types of atoms of a particular element are present, the X-ray absorption spectral shape is a linear combination of all individual sites. This aspect limits the usefulness of XANES and EXAFS in systems with atoms in a variety of sites. This problem can be partly solved with the use of selective XAFS as will be discussed in section VII.

The X-ray absorption spectral shape is described with the Fermi Golden rule

$$
\mathrm{I}_{\mathrm{XAS}} \sim\left|\left\langle\Phi_{\mathrm{f}}|\hat{\mathrm{e}} \mathrm{r}| \Phi_{\mathrm{i}}\right\rangle\right|^{2} \delta_{\mathrm{E}_{\mathrm{f}}-\mathrm{E}_{\mathrm{i}}-\hbar \omega}
$$

The intensity $\mathrm{I}_{\mathrm{XAS}}$ is proportional to a dipole matrix element (êr) coupling the initial state $\left(\Phi_{\mathrm{i}}\right)$ and the final state $\left(\Phi_{\mathrm{f}}\right)$. The delta function $(\delta)$ takes care of the conservation of energy. In the final state, a core electron has been excited. It can be described as the initial state with a continuum el ectron $(\epsilon)$ added and a core el ectron removed, i.e., $\Phi_{\mathrm{f}}=c \epsilon \Phi_{\mathrm{i}}$.

$$
\mathrm{I}_{\mathrm{XAS}} \sim\left|\left\langle\Phi_{\mathrm{i}} \underline{\mathrm{C}} \epsilon|\hat{\mathrm{e}} \mathrm{r}| \Phi_{\mathrm{i}}\right\rangle\right|^{2} \delta_{\mathrm{E}_{\mathrm{f}}-\mathrm{E}_{\mathrm{i}}-\hbar \omega}
$$

An important approximation is to assume that the matrix element can be rewritten into a single-el ectron matrix element by removing all electrons that are inactive in the transition itself; in other words, the initial state minus the core hole is removed from eq 1b. All el ectron rearrangements that take place when a core hole is excited to a continuum electron are neglected, the series of delta functions identifies with the density of states $(\rho)$, and the X-ray absorption spectral shape becomes ${ }^{82}$

$$
\mathrm{I}_{\mathrm{XAS}} \sim|\langle\epsilon|\hat{\mathrm{e}} \mathrm{r}| \mathrm{C}\rangle|^{2} \cdot \rho
$$

The dipole matrix element dictates that the density of states has an orbital moment that differs by 1 from the core state $(\Delta \mathrm{L}= \pm 1)$, while the spin is conserved $(\Delta S=0)$. In the case of $X$-rays, the quadrupole transitions are some hundred times weaker then the dipole transitions and can be neglected in most cases. They will be discussed for the preedge region in section II.B. Because in the final state a core hole is present, the density of states should be calculated with a core hole present. In many cases, this approximation gives an adequate simulation of theXAS spectral shape.

\section{1 s Edge X-ray Absorption}

1s X-ray absorption spectra can be accurately described with single-particle methods within density functional theory. This is in many case true both for metal and ligand $\mathrm{K}$-edge and band structure methods or multiple scattering calculations can be used. Following the final state rule, ${ }^{117}$ one calculates the distribution of empty states in the final state of the absorption process. Because band structure calculations are carried out in reciprocal space, it is involved to calculate the final state density of states. It would imply the use of (large) supercells to include the core hole. Therefore, the ground-state density of states is often compared directly with the X-ray absorption spectrum. Various methods exist to calculate the single-particle density of states, for example, LMTO, LSW, LAPW, and KKR. ${ }^{126}$

A formally closely related though conceptually different method is the calculation of the X-ray absorption cross section by a real-space multiple scattering cal culation. Multiple scattering is particularly appropriate for the calculation of the empty states that can be easily cal culated for arbitrary large energies. Multiple scattering calculations are usually performed with the Green function approach, with the X-ray absorption cross section written as a correlation function. The Green function describes the propagation of the electron in the solid, which is scattered by the atoms surrounding the absorbing atom. Detailed accounts of the multiple scattering are given in refs 78, 84, 93, and 118.

Because multiple scattering is performed as a realspace cluster calculation, the calculations can relatively easily be carried out for any system, ordered orystals but al so for surfaces, interfaces, enzymes, etc. In addition, the core hole potential on the absorbing atom can be added directly in multiple scattering. Another advantage of multiple scattering is that it can be performed in steps of growing cluster sizes, and it is an appealing picture to observe the changes in the theoretical spectra from the effects of increas- 
ing the number of backscatterers or from varying the included scattering paths around the absorbing atom. It has been shown that the multiple scattering formalism and the band structure formalism indeed give equivalent results if worked out rigorously in their mathematical basis. For comparison to experiment, both band structure calculations and multiple scattering results will be treated on the same footing using the density of states picture for guidance.

\section{A. Metal 1s X-ray Absorption Spectra}

The 1s edges of the $3 d$ transition metals have energies ranging from about 4 to $9 \mathrm{keV}$. They are often used for EXAFS analysis, and al so their XANES spectra are often used in many applied research investigations. An overview of 1s XANES spectra of $3 d$ transition-metal systems is given in ref 29. Metal 1s XANES spectra are traditionally interpreted with multiple scattering methods. Self-consistent full potential multiple scattering calculations have recently been carried out for $\mathrm{TiO}_{2}$ by Cabaret and co-workers. ${ }^{20}$ They show that with polarization-dependent calculations the complete spectral shape of $\mathrm{TiO}_{2}$ is nicely reproduced. In particular, the preedge region is also described correctly. It can be concluded that the 1s edges of $3 d$ transition metals do correspond to the metal p-projected density of states.

In most applications, the systems studied are not well-known. The metal 1s edges do show fine structure and edge shifts that can be used without the explicit calculation of the density of states. Figures 1 and 2 show a number of Fe 1s XANES spectra from

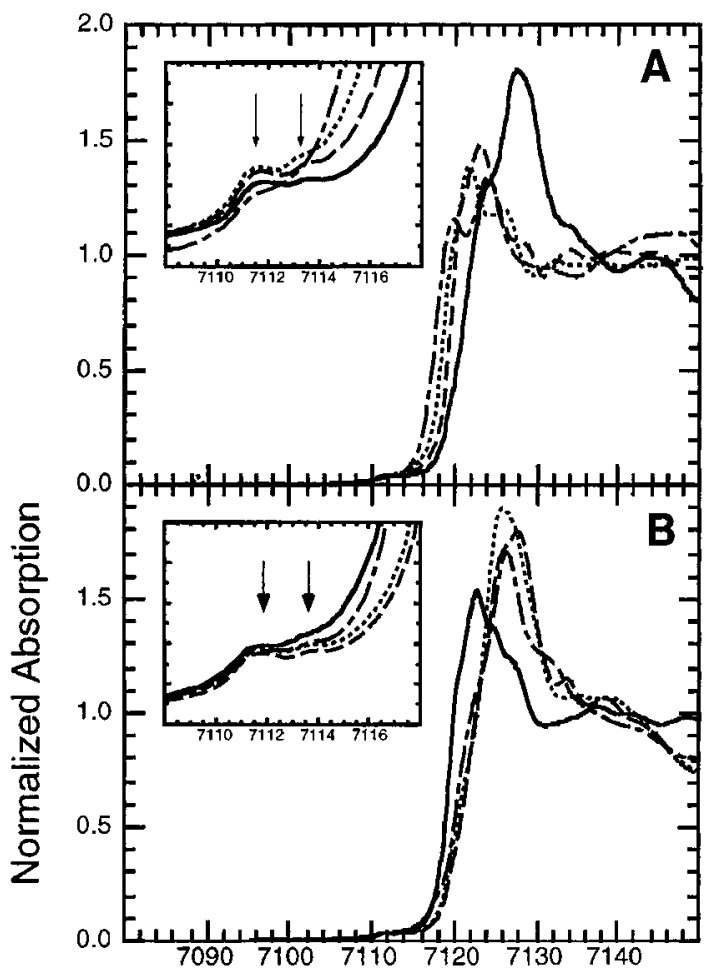

Figure 1. FeK-edge XAS spectra of octahedral high-spin $\mathrm{Fe}^{\mathrm{ll}}$ complexes. At the top are given $(\mathrm{A}) \mathrm{FeF}_{2}(-), \mathrm{FeCl}_{2}$ $(---), \mathrm{FeBr}_{2}(\cdot \cdot)$, and $\mathrm{Fel}_{2}(-\cdot-)$ and $(\mathrm{B})$ rinneite $(-)$, [Fe$\left.\left(\mathrm{H}_{2} \mathrm{O}\right)_{6}\right]\left[\mathrm{SiF}_{6}\right](---)$, [ $\left.\mathrm{Fe}\left(\mathrm{H}_{2} \mathrm{O}\right)_{6}\right]\left(\mathrm{NH}_{4}\right)_{2}\left(\mathrm{SO}_{4}\right)_{2}(\cdot \cdot)$, and $[\mathrm{Fe}$ (imidazole $\left.)_{6}\right] \mathrm{Cl}_{2}(-\cdot-)$. The insets show expanded views of the $1 \mathrm{~s} 3 \mathrm{~d}$ preedge region. (Reprinted with permission from ref 124. Copyright 1997 American Chemical Society.)

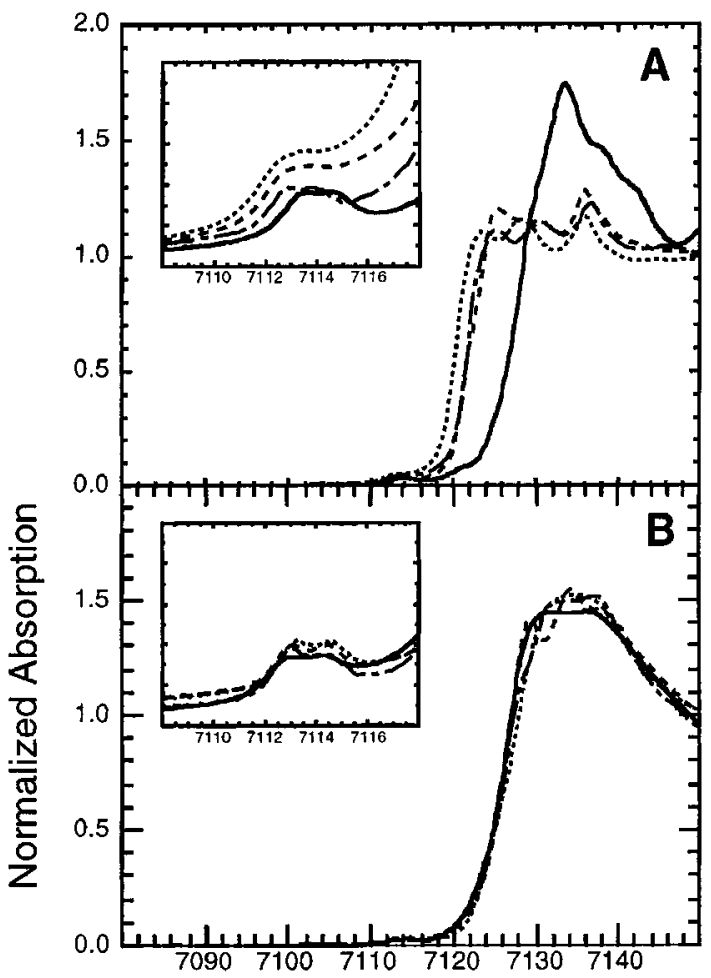

Figure 2. Fe K-edge XAS spectra of octahedral high-spin $\mathrm{Fe}^{\mathrm{lll}}$ complexes. Fe K-edge spectra of $(\mathrm{A}) \mathrm{FeF}_{3}(-), \mathrm{FeCl}_{3}$ $(---), \mathrm{FeBr}_{3}(\cdot \cdot)$, and $\left[\mathrm{FeCl}_{6}\right]\left[\mathrm{Co}\left(\mathrm{NH}_{3}\right)_{6}\right](-\cdot-)$ and $(\mathrm{B}) \mathrm{Fe}-$ $(\text { acac })_{3}(-)$, $\left(\mathrm{NH}_{4}\right)_{3} \mathrm{Fe}(\text { mal onate })_{3}(---)$, $\left[\mathrm{Fe}\left(\mathrm{H}_{2} \mathrm{O}\right)_{6}\right]\left(\mathrm{NH}_{4}\right)-$ $\left(\mathrm{SO}_{4}\right)_{2} \cdot 6 \mathrm{H}_{2} \mathrm{O}(\cdot \cdot \cdot)$, and $\mathrm{Fe}(\text { urea })_{6}\left(\mathrm{ClO}_{4}\right)_{3}(-\cdot-)$. The insets show expanded views of the 1s3d preedge region. (Reprinted with permission from ref 124. Copyright 1997 American Chemical Society.)

Westre and co-workers. ${ }^{124}$ The $\mathrm{Fe}^{\mathrm{l}}$ and Fe $\mathrm{F}^{\mathrm{Il}}$ halide spectra given at the top do show significant variations within a certain valence. Analysis of unknown iron complexes can be carried out by comparison to spectra of known systems. The Fe $\mathrm{e}^{\prime \prime \prime}$ edges are shifted to higher energy with respect to the $\mathrm{Fe}^{\|}$edges. The shift to higher energies with higher valence is a general phenomenon that can be used to determine the valence of $3 d$ transition metals in unknown systems.

\section{Metal 1s Edge Shifts}

A nice example of the use of the metal 1s edge shifts was recently given by Bae and co-workers. ${ }^{4}$ They did experiments on iron porphyrins adsorbed on carbon in acid electrolytes and follow the iron $\mathrm{K}$ edge as a function of the potential. Figure 3 shows the iron $\mathrm{K}$-edge spectra and $\mathrm{F}$ igure 4 plots the shifts in the edge energy. The intact iron porphyrin shows an edge jump of $2.0 \mathrm{eV}$, indicating a sudden valence change. ${ }^{4}$

\section{B. The Preedge Region}

The preedge region of the transition-metal 1s edges has led to a number of debates regarding the quadrupole and/or dipole nature and possible excitonic effects. The preedge region is related to transitions to the $3 d$ bands. Both direct 1s3d quadrupole transitions and dipole transitions to $4 p$ character hybridized with the $3 d$ band are possible. For the quadrupole transitions, the matrix elements are only about $1 \%$ of the dipole transition, but on the other hand, 


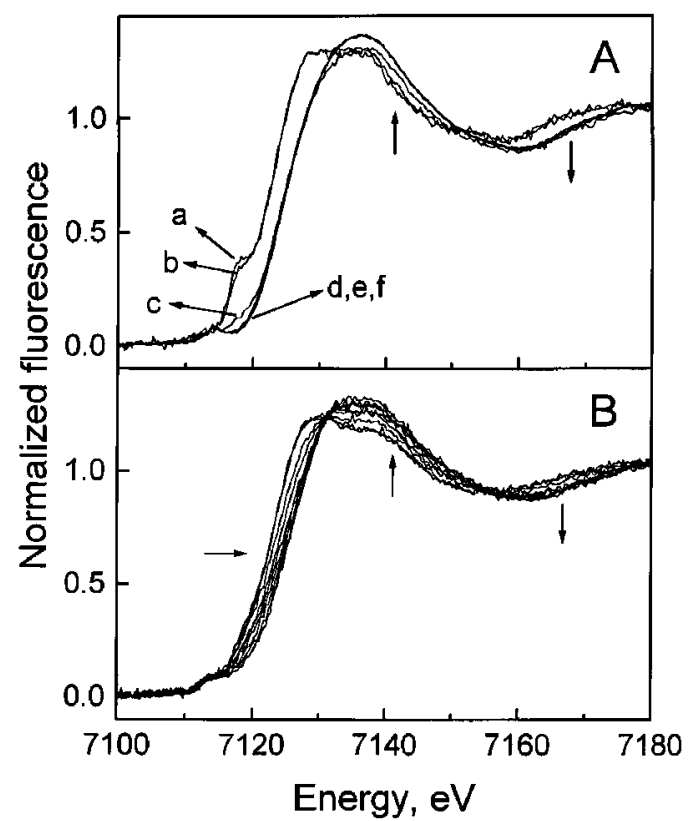

Figure 3. Series of in situ Fe K-edge XANES for intact iron porphyrin at different potentials $(A)$ and heat-treated FeTMPPCI/BP (B). (Reprinted with permission from ref 4. Copyright 1998 American Chemical Society.)

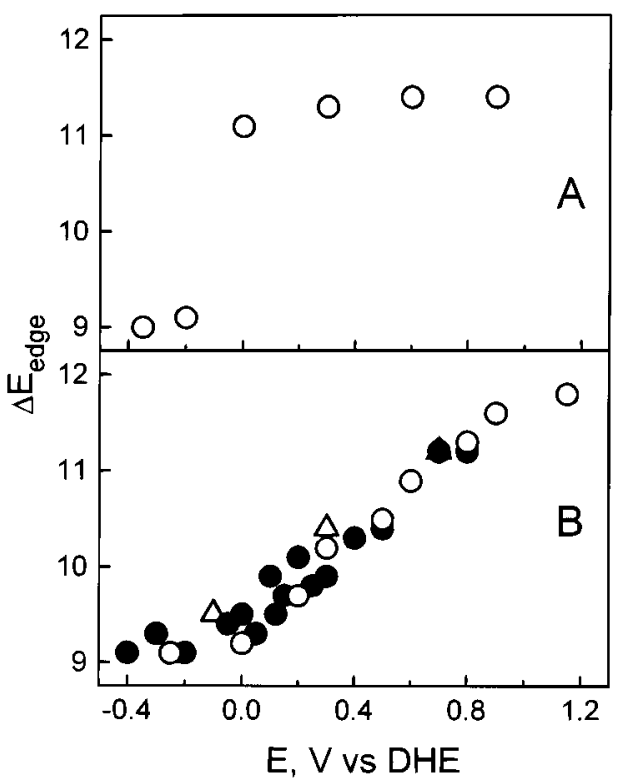

Figure 4. Plots of the edge energy versus the potential extracted from data of the type shown in Figure 3. (Reprinted with permission from ref 4. Copyright 1998 American Chemical Society.)

the amount of $3 d$ character is by far larger than the $p$ character. This can make, depending on the particular system, the contributions of quadrupole and dipole transitions equivalent in intensity. A direct manner to check the nature of the transitions is to measure the polarization dependence, which is different for quadrupole and dipole transitions. The multiple scattering results of $\mathrm{TiO}_{2}$ reproduce the three prepeaks that are caused by two effects: (1) the crystal-field splitting between the $t_{2 g}$ and $e_{g}$ orbitals and (2) the core hole effect on the quadrupole peaks. $10,13,14,21$

The preedge region of iron compounds has systematically been investigated by Westre and co-work- ers. ${ }^{124}$ Some spectra are given in Figures 1 and 2. The preedges are analyzed in terms of quadrupole transitions, and analysis showed that the spectra should be interpreted in terms of multiplet theory, including the crystal field and the atomic interactions in LS coupling. This is an approximation to the multiplet calculations as carried out in ligand-field multiplet theory, as will be explained in section III. Figure 5 shows the effect if, in addition to the crystal field and LS coupling, the $3 d$ spin-orbit coupling is included also. The Fe ${ }^{\prime \prime \prime}$ spectra are calculated from the quadrupole transitions of $3 d^{5}$ to $1 s^{1} 3 d^{6}$. The Fe ${ }^{11}$ spectra use $3 d^{6}$ as the ground state. It can be concluded that the calculations of Westre contain the essence of the spectral shapes and that the effects of 3d spin-orbit coupling are small in the preedge region. In addition, it can be shown that charge transfer (explained in section III.C.) hardly affects the spectral shape. In contrast, the effects of the spin-orbit coupling and charge transfer are very large for the $2 p$ edges, as will be discussed below. That the preedge region in $\mathrm{Fe}_{2} \mathrm{O}_{3}$ is al most completely of quadrupole nature can be shown from 1s2p resonant X-ray emission experiments that will be discussed in section IV.D

The intensity of the preedge region is much larger for compounds in which the metal site has tetrahedral symmetry than for (distorted) octahedral systems. In tetrahedral systems, the local mixing of $p$ and d nature is symmetry allowed, while for a system with inversion symmetry such as octahedral symmetry, it is 'forbidden'. This rule is relaxed in the solid, and if the density of states is calculated, one finds a small admixture of $p$ states into the $3 d$ band also for cubic systems such as $\mathrm{SrTiO}_{3} .{ }^{62}$ This admixture is less than for tetrahedral systems, which explains the small preedge. If an octahedral metal site is distorted then, depending on the particular distortion, more $p$ character will be mixed into the $3 \mathrm{~d}$ band. The result is that a distortion of the octahedron will show up as an increased intensity of the 'preedge' peak (s). That this is indeed the case has been shown by Waychunas and co-workers for a series of minerals. ${ }^{122}$ They show a roughly linear relationship between the bond angle variance (a measure of the distortion) and the preedge intensity relative to the step. In a recent paper of Arrio and co-workers, ${ }^{3}$ the preedges are calculated with multiplet theory in low symmetry, allowing for the explicit inclusion of the coupling of the dipole and quadrupole transitions. A number of iron mineral preedge spectra are divided into their dipole and quadrupole nature.

\section{Ligand 1s X-ray Absorption}

Oxygen, carbon, and nitrogen 1s X-ray absorption spectra have excitation energies between 300 and 700 $\mathrm{eV}$, which is in the soft X-ray range. The interpretation of $1 s$ spectra in the soft $X$-ray range has been dominated by band structure approaches because the details of the potential are crucial and until recently multiple scattering calculations did not reach good agreement. Figure 6a shows the density of states of rutile $\mathrm{TiO}_{2} \cdot{ }^{35}$ The total density of states has been separated into its oxygen and titanium contributions. The zero of energy is the top of the oxygen $2 p$ valence band, which can be seen to have a significant titanium contribution. The two sharp peaks between 

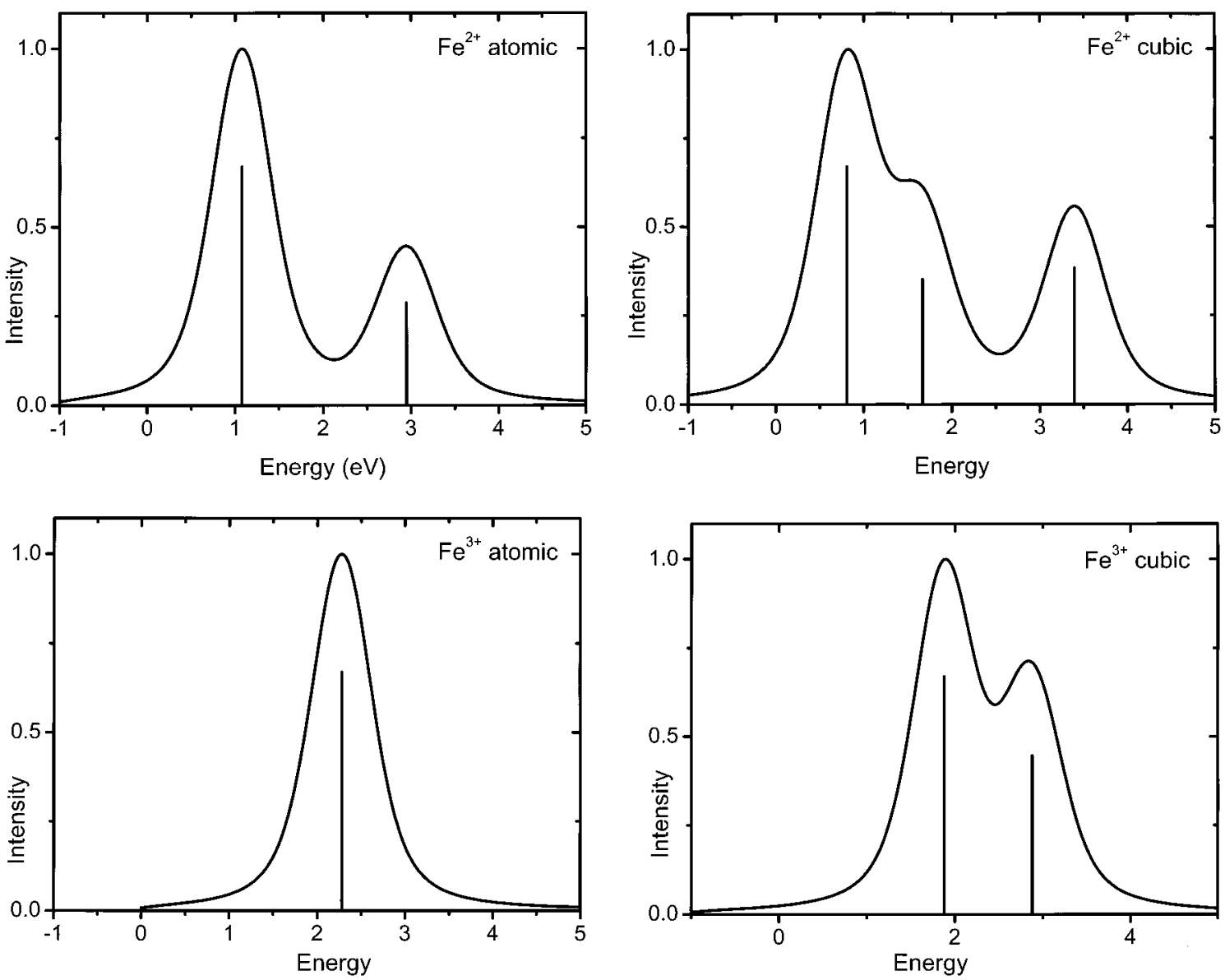

Figure 5. Atomic and ligand-field multiplet calculations for the quadrupole transitions $3 d^{5} \rightarrow 1 s^{13} d^{6}$ for Fe $e^{\prime \prime \prime}$ (bottom) and $3 d^{6} \rightarrow 1 s^{1} 3 d^{7}$ for $\mathrm{Fe}^{l 1}$ (top). The left spectra are the atomic multiplets; the right spectra have an additional cubic crystal field of $1.0 \mathrm{eV}$.
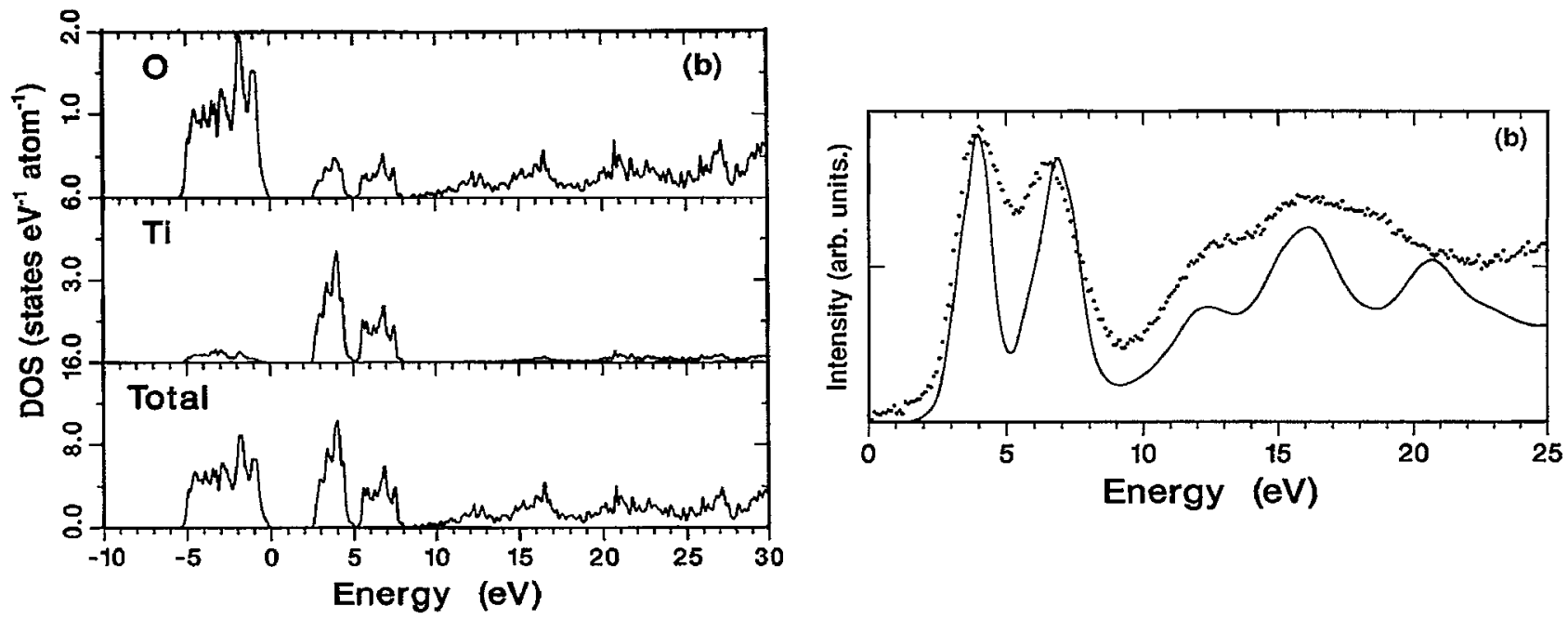

Figure 6. Right: comparison of the oxygen K-edge X-ray absorption spectra (...) with the oxygen p-projected density of states (-) for rutile $\mathrm{TiO}_{2}$; left: unbroadened density of states of rutileTiO 2 . (Reprinted with permission from ref 35. Copyright 1993 American Physical Society.)

3 and $8 \mathrm{eV}$ are the titanium $3 \mathrm{~d}$ peaks. They relate to, respectively, the $t_{2 g}$ and $e_{g}$ orbitals that are split by the cubic crystal field. Both have a significant oxygen contribution, indicating the covalent nature of $\mathrm{TiO}_{2}$. The structures at $10 \mathrm{eV}$ and higher are the titanium $4 s$ and $4 p$ bands plus all higher lying continuum states. These delocalized states have a similar density of states for oxygen and titanium. Figure $6 \mathrm{~b}$ compares the oxygen p-projected density of states with the 1s X-ray absorption spectra of rutile $\mathrm{TiO}_{2}$. The spectral shape is reproduced, and if the broadening is further optimized, a close to perfect fit of the experiment can be obtained. It can be concluded that in $\mathrm{TiO}_{2}$ the density of states as obtained from a ground-state calculation gives an accurate description of the oxygen 1s X-ray absorption spectral shape. This implies that the core hole potential does not have a large influence on the spectral shape. 


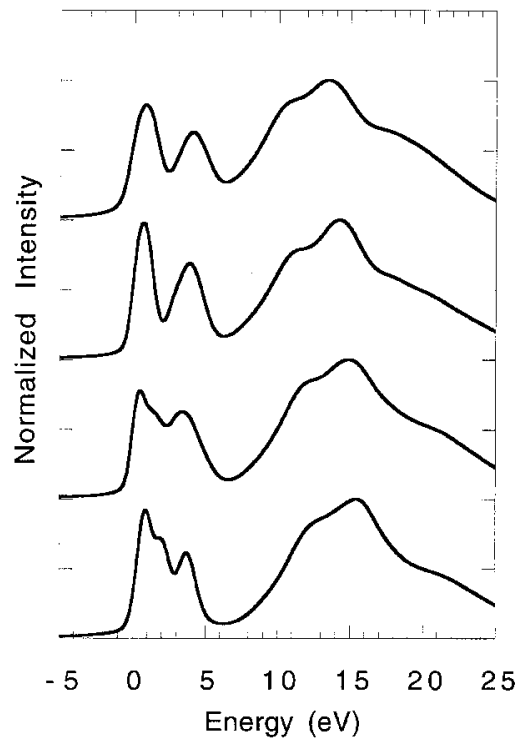

Figure 7. Series of oxygen p-projected density of states calculations of (from top to bottom ) $\mathrm{TiO}_{2}, \mathrm{VO}_{2}, \mathrm{CrO}_{2}$, and $\mathrm{MnO}_{2}$ in their (magnetic) ground states.

Figure 7 shows the oxygen p-projected density of states of, from top to bottom, $\mathrm{TiO}_{2}, \mathrm{VO}_{2}, \mathrm{CrO}_{2}$, and $\mathrm{MnO}_{2}$. The calculations have been carried out with the LMTO code without core hole and with the oxides in their magnetic ground-state configuration. ${ }^{28}$ The $\mathrm{TiO}_{2}$ density of states is the same as given in Figure 6 with some additional broadening. In the series from $\mathrm{TiO}_{2}$ to $\mathrm{MnO}_{2}$, each time one el ectron is added to the $3 d$ band that is empty for $\mathrm{TiO}_{2}$. Each added $3 d$ electron fills a $t_{2 q}$-up state. $\mathrm{VO}_{2}$ has a $t_{2 q}{ }^{1}$ configuration, $\mathrm{CrO}_{2}$ a $\mathrm{t}_{2 \mathrm{~g}}{ }^{2}$ configuration, and $\mathrm{MnO}_{2}$ a $\mathrm{t}_{2 \mathrm{~g}}{ }^{3}$ configuration. The interatomic couplings of these $t_{2 g}$ electrons gives rise to a phase transition for $\mathrm{VO}_{2}$, a half-metallic ferromagnetic system for $\mathrm{CrO}_{2}$, and a magnetic ground state for $\mathrm{MnO}_{2}$. The region between 0 and $5 \mathrm{eV}$ is related to the empty $3 \mathrm{~d}$ states. A $3 \mathrm{~d}^{0}$ ground state leaves all $103 d$ el ectrons empty. They are split by the cubic crystal field that is about 2.5 $\mathrm{eV}$ for all these dioxides. The $\mathrm{t}_{2 \mathrm{~g}}{ }^{1}$ configuration in $\mathrm{VO}_{2}$ implies that one of the six $t_{2 g}$ holes is filled, and compared with $\mathrm{TiO}_{2}$ only a sharper first peak is visible. In $\mathrm{CrO}_{2}$ with its $\mathrm{t}_{2 \mathrm{~g}}{ }^{2}$ configuration, the two filled 3d electrons have a significant exchange interaction with each other, implying an energy difference between spin-up and spin-down states of about 1.5 $\mathrm{eV}$. This is seen in the spectral shape as a split of the first peak. $\mathrm{MnO}_{2}$ has a $\mathrm{t}_{2 \mathrm{~g}}{ }^{3}$ configuration with an increased splitting between spin-up and spin-down. In this case the three visible structures are related to, respectively, $t_{2 g}$ (down), $e_{g}(u p)$, and $e_{g}$ (down). It can be seen that the spectral shape between 10 and 20 $\mathrm{eV}$ is equivalent for all oxides. This region of the spectrum is related to the 4sp and continuum density of states and reflects the equivalent crystal structures, i.e., all dioxides have a rutile crystal structure.

Comparison with experiment ${ }^{39}$ shows excellent agreement for all these oxides, and it can be concluded that the oxygen 1s X-ray absorption spectra are described well with the oxygen p-projected density of states. Some papers argue that oxygen is spectra are susceptible to multiplet effects, ${ }^{116}$ as far as the $3 \mathrm{~d}$ part of the spectrum is concerned. The good agreement of the density of states with experiments shows, however, that multiplet effects are hardly (or not at all) visible in the spectrum.

For other ligands such as carbides, nitrides, and sulfides, a similar interpretation as that for oxides is expected to hold. Their ligand 1s X-ray absorption spectra are expected to show cl ose comparison to the projected density of states, as confirmed by calculations. $24,30,99$

\section{III. $2 p$ Edge X-ray Absorption}

In the case of $2 p$ X-ray absorption ( $L$ edges), the single-particle approximation breaks down and the XANES structures are affected by the core hole wave function. The core hole and free electron of eq $1 \mathrm{~b}$ are rewritten to a $2 p$ core hole (2p) and a $3 d$ electron. All valence electrons except the $3 d$ electrons are omitted, and this yields eq $2 \mathrm{~b}$, which is the starting point for all calculations.

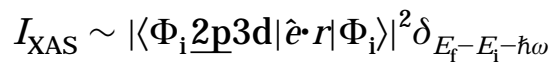

$$
\begin{aligned}
& I_{X A S} \sim\left|\left\langle 2 p 3 d^{N+1}|\hat{e} r| 3 d^{N}\right\rangle\right|^{2} \delta_{E_{f}-E_{i}-\hbar \omega}
\end{aligned}
$$

This so-called multiplet effect will be discussed in some detail because it is an important effect for all $2 p$ X-ray absorption, the 1 s preedges, as well as all $\mathrm{X}$-ray emission experiments.

The Multiplet Effect. The breakdown of the single-particle model is important in all systems with electrons in narrow bands, for example, the $3 d$ metals and the rare-earth metals. In these systems the $p$ and $\mathrm{d}$ core wave functions strongly overlap with the $3 d$ and $4 \mathrm{f}$, respectively, valence wave functions. This strong local interaction has to be considered in order to obtain an accurate description of the XANES spectral shape. A successful analysis method has been developed based on a ligand-field multiplet model. ${ }^{29}$ It offers large sensitivity to the valence and the symmetry of the element studied.

\section{A. Atomic Multiplet Theory}

The basic starting point for a large part of the analysis of core-level spectra is atomic multiplet theory. ${ }^{25,123}$ Faced with the situation of a solid with its combination of extended valence states and a localized core hole, the problem is to find the best way to treat the X-ray absorption process. A localized approach makes it possible to treat the core hole in detail, while an itinerant approach is in most cases the best approach to the ground state of the solid. In the following some aspects of atomic multiplet theory that are important for the analysis of X-ray absorption and X-ray emission spectra will be briefly introduced.

It has been shown over the last 30 years that a completely local ized approach, based on atomic multiplet theory, can be very fruitful to core-level spectroscopy. In this section atomic multiplet theory will be devel oped. Some basic quantum-mechanic results of atomic theory will be partly repeated, but the focus will be on the specific problems of a core hole in a solid. 


\section{The Atomic Hamiltonian}

The atomic Hamiltonian is given by the Schrödinger equation for an N-electron atom. The Schrödinger equation consists of the kinetic energy of the $\mathrm{N}$ electrons $\left(\sum_{\mathrm{N}} \mathrm{p}_{\mathrm{i}}^{2} / 2 \mathrm{~m}\right)$, the electrostatic interaction of the $\mathrm{N}$ electrons with the nucleus of charge $+Z$ $\left(\Sigma_{N}-Z e^{2} / r_{i}\right)$, the electron-electron repulsion $\left(H_{e e}=\right.$ $\left.\sum_{\text {pairs }} \mathrm{e}^{2} / r_{\mathrm{ij}}\right)$, and the spin-orbit coupling of each electron $\left.\left(\mathrm{H}_{\mathrm{Is}}=\sum_{\mathrm{N}} \xi\left(r_{\mathrm{i}}\right)\right)_{\mathrm{i}} \cdot \mathrm{s}_{\mathrm{i}}\right)$. The overall Hamiltonian reads

$$
H=\sum_{N} \frac{p_{i}^{2}}{2 m}+\sum_{N} \frac{-Z e^{2}}{r_{i}}+\sum_{\text {pairs }} \frac{e^{2}}{r_{i j}}+\sum_{N} \zeta\left(r_{i}\right) l_{i} \cdot s_{i}
$$

The kinetic energy and the interaction with the nucleus are the same for all electrons in a certain atomic configuration, for example, $3 d^{5}$ in the case of $M n^{\prime \prime}$. They define the average energy of a certain state $\left(\mathrm{H}_{\mathrm{av}}\right)$. This leaves the electron-electron repulsion and the spin-orbit coupling as the important interactions. The basic difficulty when solving the Schrödinger equation is that $\mathrm{H}_{\mathrm{ee}}$ is too large to be treated as a perturbation. This problem has been solved with the central fiedd approximation, in which the spherical average of the el ectron-electron interaction is separated from the nonspherical part. The spherical average $\left\langle\mathrm{H}_{\mathrm{ee}}\right\rangle$ is added to $\mathrm{H}_{\mathrm{av}}$ to form the average energy of a configuration. In the modified electron-electron Hamiltonian $\mathrm{H}^{\prime}$ ee, the spherical average has been subtracted.

$$
H^{\prime}{ }_{e e}=H_{e e}-\left\langle H_{e e}\right\rangle=\sum_{\text {pairs }} \frac{e^{2}}{r_{i j}}-\left\langle\sum_{\text {pairs }} \frac{e^{2}}{r_{i j}}\right\rangle
$$

This leaves the two interactions $\mathrm{H}^{\prime}$ ee and $\mathrm{H}_{\mathrm{ls}}$ to determine the energies of the various electronic symmetry configurations within the atomic configuration.

\section{Term Symbols}

Electronic symmetry configurations are indicated with their orbital moment $L$, spin moment $S$, and total moment $\mathrm{J}$. Together, these three quantum numbers indicate a certain state and determine its specific energy. If spin-orbit coupling is neglected, all configurations with the same $L$ and $S$ moments have the same energy. A specific configuration is indicated with a so-called term symbol ${ }^{2 S+1} \mathrm{~L}_{\text {J }}$. A term symbol gives all three quantum numbers and as such determines the symmetry and energy of a certain configuration. A single 1s electron has an orbital moment $L=0$, a spin moment $S=1 / 2$, and a total moment $\mathrm{J}=1 / 2$. This is written with a term symbol as ${ }^{2} S_{1 / 2}$. A $2 p$ el ectron has two possible symmetries depending on the spin-orbit coupling, ${ }^{2} \mathrm{P}_{1 / 2}$ and ${ }^{2} \mathrm{P}_{3 / 2}$. These two configurations are related to, respectively, the $L_{2}$ and the $L_{3}$ edge in $2 p X$-ray absorption. In the case of a transition-metal ion, the important initial state configurations are $3 \mathrm{~d}^{\mathrm{N}}$. In the final state with a $3 s$ or a $3 p$ core hole, the important configurations are $3 s^{1} 3 d^{N}$ and $3 p^{5} 3 d^{N}$. The main quantum number does not influence the coupling schemes, so exactly the same term symbols can be found for $4 d$ and $5 d$ systems and for $2 \mathrm{~s}$ and $2 \mathrm{p}$ core holes.

A $3 d^{1}$ configuration is 10 -fold degenerate and has the term symbols ${ }^{2} D_{5 / 2}$ and ${ }^{2} D_{3 / 2}$ with, respectively, six and four electrons. The degeneracy of a term symbol ${ }^{2 S}{ }^{+1} L_{j}$ is given as $2 J+1$. The overall degeneracy of a term symbol ${ }^{2 S+1} \mathrm{~L}$ is $(2 S+1)(2 \mathrm{~L}+$ $1)$. The LS term symbols of a $3 d^{1} 4 d^{1}$ configuration can be found by multiplying ${ }^{2} \mathrm{D} \otimes^{2} \mathrm{D}$. The multiplication of $L$ and $S$ is independent, and one finds that the total orbital moment $L$ is equal to $0,1,2,3$, or 4 . $\mathrm{S}$ is equal to 0 or 1 . This gives the term symbols as

\begin{tabular}{|c|c|c|c|c|c|c|c|c|c|c|c|}
\hline $3 d^{1} 4 d^{1}$ & ${ }^{15}$ & ${ }^{1} \mathrm{P}$ & ${ }^{1} \mathrm{D}$ & ${ }^{1} \mathrm{~F}$ & ${ }^{1} \mathrm{G}$ & ${ }^{3} \mathrm{~S}$ & ${ }^{3 p}$ & ${ }^{3} \mathrm{D}$ & ${ }^{3} \mathrm{~F}$ & ${ }^{3} \mathrm{G}$ & $\Sigma$ \\
\hline degen. & 1 & 3 & 5 & 7 & 9 & 3 & $\begin{array}{l}9 \\
0\end{array}$ & $\begin{array}{r}15 \\
1\end{array}$ & $\begin{array}{r}21 \\
2\end{array}$ & $\begin{array}{r}27 \\
3\end{array}$ & 100 \\
\hline J values & 0 & 1 & 2 & 3 & 4 & 1 & $\begin{array}{l}1 \\
2\end{array}$ & $\begin{array}{l}2 \\
3\end{array}$ & $\begin{array}{l}3 \\
4\end{array}$ & $\begin{array}{l}4 \\
5\end{array}$ & \\
\hline
\end{tabular}
indicated in Table 1. The total degeneracy of the

$3 d^{1} 4 d^{1}$ configuration is 100 , which can be checked by adding the degeneracies of the 10 term symbols. After inclusion of spin-orbit coupling, a total of $18^{2 S+1} L_{\text {j }}$ term symbols is found.

A $3 d^{2}$ configuration does not have a degeneracy of 100. The Pauli exclusion principle forbids two $3 d$ electrons to have the same quantum numbers, which has the consequence that the second el ectron has not 10 but only 9 possible states. In addition, the sequence of the quantum states for the two electrons make no difference, which divides the number of different two-electron states by two. This give $10 \times$ $9 / 2=45$ possible states. One can write out all 45 combinations of a $3 d^{2}$ configuration and sort them by their $M_{L}$ and $M_{S}$ quantum numbers. Analysis of the combinations of the allowed $M_{L}$ and $M_{S}$ quantum numbers yields the term symbols ${ }^{1} \mathrm{G},{ }^{3} \mathrm{~F},{ }^{1} \mathrm{D},{ }^{3} \mathrm{P}$, and ${ }^{1} S$. This is a subset of the term symbols of a $3 d^{1} 4 d^{1}$ configuration. The term symbols can be divided into their $\mathrm{J}$ quantum numbers as ${ }^{3} \mathrm{~F}_{2},{ }^{3} \mathrm{~F}_{3},{ }^{3} \mathrm{~F}_{4},{ }^{3} \mathrm{P}_{0},{ }^{3} \mathrm{P}_{1}$, ${ }^{3} \mathrm{P}_{2},{ }^{1} \mathrm{G}_{4},{ }^{1} \mathrm{D}_{2}$, and ${ }^{1} \mathrm{~S}_{0}$. In the case of a $3 \mathrm{~d}^{3}$ configuration, a similar approach gives that the possible spin states are doublet and quartet. The quartet states have all spins parallel, and the Pauli principle implies that there are two quartet term symbols, respectively, ${ }^{4} \mathrm{~F}$ and ${ }^{4} \mathrm{P}$. The doublet states have two electrons parallel, and for these two electrons, the Pauli principle yields the combinations identical to the triplet states of the $3 d^{2}$ configuration. To these two parallel electrons a third electron is added antiparallel, where this third electron can have any value of its orbital quantum number $m_{1}$. Writing out all combinations and separating them into the total orbital moments $M_{1}$ gives the doublet term symbols ${ }^{2} \mathrm{H},{ }^{2} \mathrm{G},{ }^{2} \mathrm{~F},{ }^{2} \mathrm{D}$, another ${ }^{2} \mathrm{D}$, and ${ }^{2} \mathrm{P}$. By adding the degeneracies, it can be checked that a $3 \mathrm{~d}^{3}$ configuration has 120 different states, i.e., $10 \times 9 / 2 \times 8 / 3$. The general formula to determine the degeneracy of 
a $3 d^{N}$ configuration is

$$
\left(\begin{array}{l}
10 \\
n
\end{array}\right)=\frac{10 !}{(10-n) ! n !}
$$

A new result with respect to the $s$ and $p$ electrons is that one finds for a $3 d^{3}$ configuration two states with an identical term symbol. To distinguish both term symbols, the so-called seniority number is introduced. The seniority number is the number $N$ of the $3 d^{N}$ configuration for which a term symbol occurs first. For example, the ${ }^{2} \mathrm{D}$ term symbol occurs for a $3 \mathrm{~d}^{1}$ configuration; hence, it has seniority number 1 . The term symbol could be rewritten as ${ }_{1}^{2} \mathrm{D}$. The second ${ }^{2} \mathrm{D}$ term symbol of $3 d^{3}$ takes its seniority number from the next lowest number $\mathrm{N}$ and is written as ${ }_{3}^{2} \mathrm{D}$. The discussion of the seniority number brings us to another observation, which is that the term symbols of a configuration $3 \mathrm{~d}^{\mathrm{N}}$ do also exist in a configuration $3 \mathrm{~d}^{\mathrm{N}+2}$. Thus, the term symbols of $3 \mathrm{~d}^{4}$ contain all term symbols of $3 d^{2}$, which contains the ${ }^{1} S$ term symbol of $3 d^{0}$. Similarly, the term symbols of $3 d^{5}$ contain all term symbols of $3 d^{3}$, which contains the ${ }^{2} \mathrm{D}$ term symbol of $3 \mathrm{~d}^{1}$. In addition, there is a symmetry equival ence of holes and electrons; hence, $3 d^{3}$ and $3 d^{7}$ have exactly the same term symbols.

An important X-ray absorption process is the $2 p$ $\rightarrow 3 d$ excitation process. The $2 p$ X-ray absorption edge is often studied for the $3 d$ transition-metal series, and it provides a wealth of information. Crucial for its understanding are the configurations of the $2 p^{5} 3 d^{N}$ final states. The term symbols of the $2 \mathrm{p}^{5} 3 \mathrm{~d}^{\mathrm{N}}$ states are found by multiplying the configurations of $3 \mathrm{~d}^{\mathrm{N}}$ with a $2 \mathrm{P}$ term symbol. The total degeneracy of a $2 p^{5} 3 d^{N}$ state is given in eq 5 . For example, a $2 p^{5} 3 d^{5}$ configuration has 1512 possible states. Analysis shows that these 1512 states are divided into 205 term symbols, implying, in principle, 205 possible final states. Whether all these final states have finite intensity depends on the selection rules.

$$
6 \times\left(\begin{array}{l}
10 \\
n
\end{array}\right)=6 \times \frac{10 !}{(10-n) ! n !}
$$

\section{The Matrix Elements}

Above found the number and symmetry of the states of a certain $3 d^{N}$ configuration were found. The next task is to cal culate the matrix elements of these states with the Hamiltonian $\mathrm{H}_{\text {ATOM. }}$. As discussed in the previous section, $\mathrm{H}_{\text {ATOM }}$ consists of the effective el ectron-electron interaction $\mathrm{H}_{\mathrm{ee}}^{\prime}$ and the spin-orbit coupling $\mathrm{H}_{\mathrm{Is}}$

$$
\mathrm{H}_{\text {ATOM }}=\sum_{\text {pairs }} \frac{\mathrm{e}^{2}}{\mathrm{r}_{\mathrm{ij}}}+\sum_{\mathrm{N}} \xi\left(\mathrm{r}_{\mathrm{i}}\right) \mathrm{I}_{\mathrm{i}} \cdot \mathrm{S}_{\mathrm{i}}
$$

The el ectron-electron interaction commutes with $\mathrm{L}^{2}$, $\mathrm{S}^{2}, \mathrm{~L}_{\mathrm{z}}$, and $\mathrm{S}_{\mathrm{z}}$, which implies that all off-diagonal elements are zero. A simple example is a $1 \mathrm{~s} 2 \mathrm{~s}$ configuration consisting of ${ }^{1} \mathrm{~S}$ and ${ }^{3} \mathrm{~S}$ term symbols. The respective energies can be shown to be

$$
\begin{aligned}
& \left\langle{ }^{1} S\left|\frac{\mathrm{e}^{2}}{\mathrm{r}_{12}}\right|{ }^{1} \mathrm{~S}\right\rangle=\mathrm{F}^{0}(1 \mathrm{~s} 2 \mathrm{~s})+\mathrm{G}^{0}(1 \mathrm{~s} 2 \mathrm{~s}) \\
& \left\langle{ }^{3} \mathrm{~S}\left|\frac{\mathrm{e}^{2}}{\mathrm{r}_{12}}\right|{ }^{3} \mathrm{~S}\right\rangle=\mathrm{F}^{0}(1 \mathrm{~s} 2 \mathrm{~s})-\mathrm{G}^{0}(1 \mathrm{~s} 2 \mathrm{~s})
\end{aligned}
$$

$\mathrm{F}^{0}$ and $\mathrm{G}^{0}$ are the Slater-Condon parameters for, respectively, the direct Coulomb repulsion and the Coulomb exchange interaction. The main result can be stated as "the singlet and the triplet state are split by the exchange interaction". This energy difference is $2 \mathrm{G}^{0}$ (1s2s). An analogous result is found for a $1 \mathrm{~s} 2 \mathrm{p}$ state for which the singlet and triplet states are split by $2 / 3 G^{0}$ (1s2p). The $2 / 3$ prefactor is determined by the degeneracy of the $2 p$ state. The general formulation of the matrix elements of two-electron wave functions can be written as

$$
\left\langle{ }^{2 S+1} L_{\jmath}\left|\frac{e^{2}}{r_{12}}\right|{ }^{2 S+1} L_{J}\right\rangle=\sum_{k} f_{k} F^{k}+\sum_{k} g_{k} G^{k}
$$

To obtain this result, the radial parts $F^{k}$ and $G^{k}$ have been separated using the Wigner-Eckardt theorem and Hamiltonian $1 / r_{12}$ has been expanded in a series. ${ }^{123}$ The angular parts $f_{k}$ and $g_{k}$ can be calculated using angular momentum coupling, and the result is written in terms of $3 j$ and $6 j$ symbols

$$
\begin{gathered}
f_{k}=\left(2 I_{1}+1\right)\left(2 I_{2}+1\right) \cdot(-1)^{L} \\
\left(\begin{array}{lll}
I_{1} & k & I_{1} \\
0 & 0 & 0
\end{array}\right)\left(\begin{array}{lll}
I_{2} & k & I_{2} \\
0 & 0 & 0
\end{array}\right)\left(\begin{array}{lll}
I_{1} & I_{2} & L \\
I_{2} & l_{1} & k
\end{array}\right) \\
g_{k}=\left(2 I_{1}+1\right)\left(2 I_{2}+1\right) \cdot(-1)^{S} \\
\left(\begin{array}{lll}
I_{1} & k & I_{2} \\
0 & 0 & 0
\end{array}\right)\left(\begin{array}{lll}
I_{1} & k & I_{2} \\
0 & 0 & 0
\end{array}\right)\left(\begin{array}{lll}
I_{1} & I_{2} & L \\
I_{2} & I_{1} & k
\end{array}\right)
\end{gathered}
$$

For equivalent electrons, $g_{k}$ is not present and $f_{k}$ can be simplified by setting $\mid=I_{1}=I_{2}$. The values of $k$ can be determined from the so-called triangl e conditions of the $3 \mathrm{j}$ symbols. The triangle conditions imply that for $3 \mathrm{j}$ symbols with all zeros for $\mathrm{m}_{\mathrm{j}}$ (given in the bottom row), the sum of the $\mathrm{j}$ values (given in the top row) must be even and that the maximum j value is equal to the sum of the two others. Using the two $3 j$ symbols of eqs 10 and 11 , this implies for $f_{k}$ that $k$ must always be even. $\mathrm{k}=0$ is always present, and the maximum value for $k$ equals two times the lowest value of $I$. For $g_{k}$ it implies that $k$ is even if $I_{1}+I_{2}$ is even and $k$ is odd if $I_{1}+I_{2}$ is odd. The maximum value of $k$ equals $I_{1}+I_{2}$. Table 2 gives the possible $k$ values

Table 2: Possible Values of $k$ for the Angular Coefficients $f_{k}$ and $g_{k}$

\begin{tabular}{llrlrrrrr}
\hline conf. & $\mathrm{f}_{\mathrm{k}}$ & $\mathrm{g}_{\mathrm{k}}$ & conf. & $\mathrm{f}_{\mathrm{k}}$ & $\mathrm{g}_{\mathrm{k}}$ & conf. & \multicolumn{1}{c}{$\mathrm{f}_{\mathrm{k}}$} & $\mathrm{g}_{\mathrm{k}}$ \\
\hline $1 \mathrm{~s}^{2}$ & 0 & & $1 \mathrm{~s} 2 \mathrm{~s}$ & 0 & 0 & $1 \mathrm{~s} 2 \mathrm{p}$ & 0 & 1 \\
$2 \mathrm{p}^{2}$ & 02 & & $2 \mathrm{p} 3 \mathrm{p}$ & 02 & 02 & $2 \mathrm{p} 3 \mathrm{~d}$ & 02 & 13 \\
$3 \mathrm{~d}^{2}$ & 024 & & 3d4d & 024 & 024 & $3 \mathrm{~d} 4 \mathrm{f}$ & 024 & 135 \\
\hline
\end{tabular}

for some important configurations.

The energies of the representations of the $3 \mathrm{~d}^{2}$ configuration are calculated. First, it can be shown that $f_{0}$ is equal to the number of permutations ( $n(n$ 
- 1)/2) of $n$ electrons, i.e., $f_{0}$ is equal to 1.0 for twoelectron configurations. The el ectrons come from the same shell; hence, there are no exchange interactions. One is left with the calculation of $f_{2}$ and $f_{4}$ for the five term symbols ${ }^{1} \mathrm{~S},{ }^{3} \mathrm{P},{ }^{1} \mathrm{D},{ }^{3} \mathrm{~F}$, and ${ }^{1} \mathrm{G} . \mathrm{I}_{1}=\mathrm{I}_{2}=2$, which implies that the prefactor $\left(2 \mathrm{I}_{1}+1\right)\left(2 \mathrm{I}_{2}+1\right)$ is equal to 25 . The $3 \mathrm{j}$ symbols are only dependent on $\mathrm{k}$ and are equal to $\sqrt{ } 2 / 35$. For all five states this gives a prefactor of $25 \times 2 / 35=10 / 7$, i.e., $f_{k}$ is equal to

$$
f_{k}=\frac{10}{7} \cdot\left\{\begin{array}{lll}
2 & 2 & L \\
2 & 2 & k
\end{array}\right\} \cdot(-1)^{L}
$$

The Slater-Condon parameters $\mathrm{F}^{2}$ and $\mathrm{F}^{4}$ have approximately a constant ratio: $\mathrm{F}^{4}=0.62 \mathrm{~F}^{2}$. The last column in Table 3 gives the approximate energies of

\begin{tabular}{|c|c|c|c|c|c|c|c|}
\hline & & $f_{2}$ & & & $f_{4}$ & & energy \\
\hline is & $\frac{10}{7} \begin{cases}2 & 2 \\
2 & 2\end{cases}$ & $\left.\begin{array}{ll}2 & 0 \\
2 & 2\end{array}\right\}$ & $2 / 7$ & $\frac{10}{7}\left\{\begin{array}{l}2 \\
2\end{array}\right.$ & $\left.\begin{array}{ll}2 & 0 \\
2 & 4\end{array}\right\}$ & $2 / 7$ & $0.46 F^{2}$ \\
\hline 3P & $-\frac{10}{7} \begin{cases}2 \\
2\end{cases}$ & $\left.\begin{array}{ll}2 & 1 \\
2 & 2\end{array}\right\}$ & $3 / 21$ & $-\frac{10}{7}\left\{\begin{array}{l}2 \\
2\end{array}\right.$ & $\left.\begin{array}{ll}2 & 1 \\
2 & 4\end{array}\right\}$ & $-4 / 21$ & $0.02 F^{2}$ \\
\hline $1 \mathrm{D}$ & $\frac{10}{7} \begin{cases}2 & 2 \\
2 & 2\end{cases}$ & $\left.\begin{array}{ll}2 & 2 \\
2 & 2\end{array}\right\}$ & $-3 / 49$ & $\frac{10}{7}\left\{\begin{array}{l}2 \\
2\end{array}\right.$ & $\left.\begin{array}{ll}2 & 2 \\
2 & 4\end{array}\right\}$ & $4 / 49$ & $-0.01 F^{2}$ \\
\hline $3 F$ & $-\frac{10}{7} \begin{cases}2 & 2 \\
2 & 2\end{cases}$ & $\left.\begin{array}{ll}2 & 3 \\
2 & 2\end{array}\right\}$ & $-8 / 49$ & $-\frac{10}{7}\left\{\begin{array}{l}2 \\
2\end{array}\right.$ & $\left.\begin{array}{ll}2 & 3 \\
2 & 4\end{array}\right\}$ & $-1 / 49$ & $-0.18 F^{2}$ \\
\hline $1 G$ & $\frac{10}{7} \begin{cases}2 & 2 \\
2 & 2\end{cases}$ & $\left.\begin{array}{ll}2 & 4 \\
2 & 2\end{array}\right\}$ & $4 / 49$ & $\frac{10}{7}\left\{\begin{array}{l}2 \\
2\end{array}\right.$ & $\left.\begin{array}{ll}2 & 4 \\
2 & 4\end{array}\right\}$ & $1 / 441$ & $0.08 \mathrm{~F}^{2}$ \\
\hline
\end{tabular}

the five term symbols. In case of the $3 d$ transitionmetal ions, $\mathrm{F}^{2}$ is approximately equal to $10 \mathrm{eV}$. For the five term symbols this gives the energies as ${ }^{3} \mathrm{~F}$ at $-1.8 \mathrm{eV},{ }^{1} \mathrm{D}$ at $-0.1 \mathrm{eV},{ }^{3} \mathrm{P}$ at $+0.2 \mathrm{eV},{ }^{1} \mathrm{G}$ at +0.8 $\mathrm{eV}$, and ${ }^{1} \mathrm{~S}$ at $+4.6 \mathrm{eV}$. The ${ }^{3} \mathrm{~F}$ term symbol has lowest energy and is the ground state of a $3 d^{2}$ system. This is in agreement with Hund's rules, which will be discussed in the next section. The three states ${ }^{1} D$, ${ }^{3} \mathrm{P}$, and ${ }^{1} \mathrm{G}$ are close in energy, $1.7-2.5 \mathrm{eV}$ above the ground state. The ${ }^{1} \mathrm{~S}$ state has a high energy of 6.3 $\mathrm{eV}$ above the ground state, the reason being that two el ectrons in the same orbit strongly repel each other.

Table 4 gives three related notations that are used

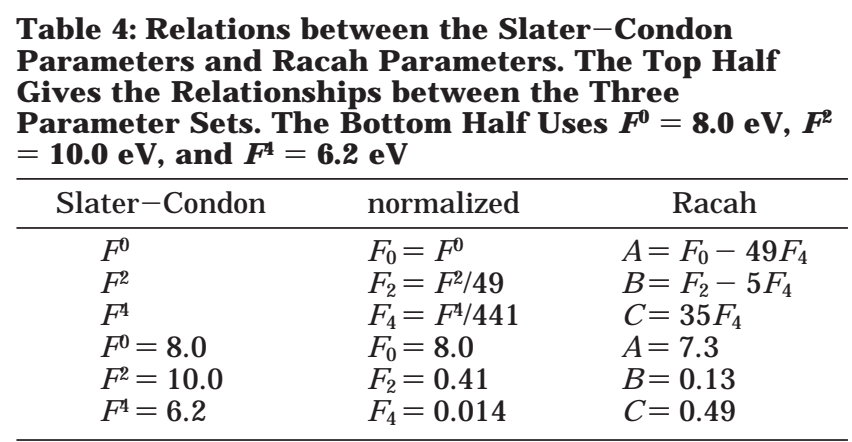

to indicate the radial integrals: the Slater-Condon parameters $F^{k}$, the normalized Slater-Condon parameters $F_{k}$, and the Racah parameters $A, B$, and $C$.
The bottom half of Table 4 uses the relationship between $\mathrm{F}^{2}$ and $\mathrm{F}^{4}$ and further uses a typical $\mathrm{F}^{2}$ value of $10 \mathrm{eV}$ and a $\mathrm{F}^{0}$ value of $8 \mathrm{eV}$. For three and more electrons, the situation is more complex. It is not straightforward to write down an antisymmetrized three-electron wave function. It can be shown that the three-electron wave function can be build from two-electron wave functions with the use of the socalled coefficients of fractional parentage. The coefficients of fractional parentage are indicated with $\mathrm{C}_{\mathrm{L}_{1} \mathrm{~S}_{1}}^{\mathrm{LS}}$. The three-electron wave function with quantum numbers LS is generated from a series of twoelectron wave functions with quantum numbers $L_{1} S_{1}$.

\section{Atomic Multiplet Ground States of $3 d^{N}$ Systems}

The ground-state symmetries of the transitionmetal compounds that are characterized with a partly filled 3d band will be discussed. The term symbols with the lowest energy are found after calculating the matrix elements. The term symbols found to be at the lowest energy are indicated in Table 5. The

\begin{tabular}{|c|c|c|c|c|}
\hline transition & $\begin{array}{l}\text { no. of term } \\
\text { symbols: } \\
\text { ground } \\
\text { state }\end{array}$ & $\begin{array}{l}\text { symmetry: } \\
\text { ground } \\
\text { state }\end{array}$ & $\begin{array}{l}\text { no. of term } \\
\text { symbols: } \\
\text { final state }\end{array}$ & $\begin{array}{c}\text { no. of } \\
\text { allowed } \\
\text { transitions }\end{array}$ \\
\hline $\begin{array}{l}3 d^{0} \rightarrow 2 p^{5} 3 d^{1} \\
3 d^{1} \rightarrow 2 p^{5} 3 d^{2} \\
3 d^{2} \rightarrow 2 p^{5} 3 d^{3} \\
3 d^{3} \rightarrow 2 p^{5} 3 d^{4} \\
3 d^{4} \rightarrow 2 p^{5} 3 d^{5} \\
3 d^{5} \rightarrow 2 p^{5} 3 d^{6} \\
3 d^{6} \rightarrow 2 p^{5} 3 d^{7} \\
3 d^{7} \rightarrow 2 p^{5} 3 d^{8} \\
3 d^{8} \rightarrow 2 p^{5} 3 d^{9} \\
3 d^{9} \rightarrow 2 p^{5} 3 d^{10}\end{array}$ & $\begin{array}{r}1 \\
2 \\
9 \\
19 \\
34 \\
37 \\
34 \\
19 \\
9 \\
2\end{array}$ & $\begin{array}{l}{ }^{1} S_{0} \\
{ }^{2} D_{3 / 2} \\
{ }^{3} F_{2} \\
{ }^{4} F_{3 / 2} \\
{ }^{5} D_{0} \\
{ }^{6} S_{5 / 2} \\
{ }^{5} D_{2} \\
{ }^{4} F_{9 / 2} \\
{ }^{3} F_{4} \\
{ }^{2} D_{5 / 2}\end{array}$ & $\begin{array}{r}12 \\
45 \\
110 \\
180 \\
205 \\
180 \\
110 \\
45 \\
12 \\
2\end{array}$ & $\begin{array}{r}3 \\
29 \\
68 \\
95 \\
32 \\
110 \\
68 \\
16 \\
4 \\
1\end{array}$ \\
\hline
\end{tabular}

finding of the ${ }^{3} \mathrm{~F}$ state as the ground state of a $3 \mathrm{~d}^{2}$ configuration is an example of the so-called Hund's rules. On the basis of experimental information, Hund did formulate three rules to determine the ground state of a $3 \mathrm{~d}^{\mathrm{N}}$ configuration. The Hund rules are as follows: (1) term symbol with maximum spin $\mathrm{S},(2)$ term symbol with maximum orbital moment $\mathrm{L}$, and (3) term symbol with maximum total moment $J$, if the shell is more than half full.

A configuration has the lowest energy if the electrons are as far apart as possible. Hund's first rule of 'maximum spin' can be understood from the Pauli principle: Electrons with parallel spins must be in different orbitals, which overall implies larger separations, hence lower energies. This is, for example, evident for a $3 d^{5}$ configuration, where the ${ }^{6} S$ state has its five electrons divided over the five spin-up orbitals, which minimizes their repulsion. In case of $3 d^{2}$, Hund's first rule implies that either the ${ }^{3 P}$ or ${ }^{3} F$ term symbol must have the lowest energy. F rom the previous section, one finds that the ${ }^{3} \mathrm{~F}$ term symbol is lower than the ${ }^{3 P}$ term symbol, the reason being that the ${ }^{3} \mathrm{~F}$ wave function tends to minimize electron repulsion. The effects of spin-orbit coupling are wellknown in the case of core states. Consider, for 
example, the $2 p$ XAS or XPS spectrum of nickel. The ${ }^{2} \mathrm{P}_{3 / 2}$ peak is positioned at approximately $850 \mathrm{eV}$ and the ${ }^{2} \mathrm{P}_{1 / 2}$ at about $880 \mathrm{eV}$, where the state with the lowest binding energy is related to the lowest energy of the configuration. The case of the $2 p$ core state is an example of $\mathrm{d} \mathrm{H}$ und's third rule: the configuration is $2 p^{5}$, which is more than half-full, implying that the highest J value has the lowest energy. The third rule implies that the ground state of a $3 d^{8}$ configuration is ${ }^{3} F_{4}$, while it is ${ }^{3} \mathrm{~F}_{2}$ in the case of a $3 d^{2}$ configuration.

\section{X-ray Absorption Spectra Described with Atomic Multiplets}

I will start with the description of closed-shell systems. The $2 p$ X-ray absorption process excites a $2 p$ core electron into the empty $3 d$ shell, and the transition can be described as $2 \mathrm{p}^{6} 3 \mathrm{~d}^{0} \rightarrow 2 \mathrm{p}^{5} 3 \mathrm{~d}^{1}$. The ground state has ${ }^{1} S_{0}$ symmetry, and it is found that the term symbols of the final state are ${ }^{1} P_{1},{ }^{1} D_{2},{ }^{1} F_{3}$, ${ }^{3} \mathrm{P}_{012},{ }^{3} \mathrm{D}_{123}$, and ${ }^{3} \mathrm{~F}_{234}$. The energies of the final states are affected by the 2p3d Slater-Condon parameters, the $2 p$ spin-orbit coupling, and the $3 d$ spin-orbit coupling. The X-ray absorption transition matrix elements to be calculated are

$$
\mathrm{I}_{\mathrm{XAS}} \propto\left\langle 3 \mathrm{~d}^{0}|\mathrm{p}| 2 \mathrm{p}^{5} 3 \mathrm{~d}^{1}\right\rangle^{2}
$$

The symmetry aspects are

$$
\mathrm{I}_{\mathrm{XAS}} \propto\left\langle\left[{ }^{1} \mathrm{~S}_{0}\right] \mid\left[{ }^{1} \mathrm{P}_{1}\right]\left[\left.\right|^{1,3} \mathrm{PDF}\right]\right\rangle^{2}
$$

The symmetry of the dipole transition is given as ${ }^{1} \mathrm{P}_{1}$, according to the dipole selection rules, which state that $\Delta \mathrm{J}=+1,0,-1$. Within LS coupling, also $\Delta \mathrm{S}$ $=0$ and $\Delta \mathrm{L}=1$. The dipole selection rule reduces the number of final states that can be reach from the ground state. The J value in the ground state is zero. In this case, the dipole selection rule proclaims that the J value in the final state must be 1 ; thus, only the three term symbols ${ }^{1} P_{1},{ }^{3} P_{1}$, and ${ }^{3} D_{1}$ can obtain finite intensity. The problem of calculating the $2 p$ absorption spectrum is reduced to solving the $3 \times 3$ energy matrix of the final states with $J=1$. As discussed above, the atomic energy matrix consists of terms related to the two-electron Slater integrals and the $2 p$ and $3 d$ spin-orbit couplings.

A series of X-ray absorption spectra of tetravalent titanium $2 p$ and $3 p$ edges and the trivalent lanthanum $3 d$ and $4 d$ edges is compared. The ground states of $\mathrm{Ti}^{\mathrm{IV}}$ and $\mathrm{L} \mathrm{a}^{\prime \prime \prime}$ are, respectively, $3 \mathrm{~d}^{\circ}$ and $4 \mathrm{f}^{0}$, and they share a ${ }^{1} S$ ground state. The transitions at the four edges are, respectively

$$
\begin{aligned}
& \mathrm{Ti}^{\mathrm{IV}} L_{2,3} \text { edge: } 3 d^{0} \rightarrow 2 p^{5} 3 d^{1} \\
& \mathrm{Ti}^{\mathrm{IV}} \mathrm{M}_{2,3} \text { edge: } 3 d^{0} \rightarrow 3 p^{5} 3 d^{1} \\
& \mathrm{La}^{\prime \prime \prime} M_{4,5} \text { edge: } 4 f^{0} \rightarrow 3 d^{9} 4 f^{1} \\
& \text { La }^{\prime \prime \prime} N_{4,5} \text { edge: } 4 f^{0} \rightarrow 4 d^{9} 4 f^{1}
\end{aligned}
$$

These four calculations are equivalent, and all spectra consist of three peaks with J $=1$ as described above in the case of the $2 p$ edge of Tilv. The things that change are the values of the atomic SlaterCondon parameters and core hole spin-orbit couplings. They are given in Table 6 for the four

Table 6: Relative Intensities, Energy, Core Hole Spin-Orbit Coupling, and $\mathbf{F}_{2}$ Slater-Condon Parameters Are Compared for Four Different ${ }^{1} \mathbf{S}_{0}$ Systems

\begin{tabular}{lcrrc}
\hline \multicolumn{1}{c}{ edge } & Ti 2p & Ti 3p & La 3d & La 4d \\
\hline average energy (eV) & 464 & 37 & 840 & 103 \\
core spin-orbit (eV) & 3.78 & 0.43 & 6.80 & 1.12 \\
$\mathrm{~F}^{2}$ Slater-Condon (eV) & 5.04 & 8.91 & 5.65 & 10.45 \\
\hline \multicolumn{5}{c}{ relative intensities } \\
\hline prepeak & $10^{-2}$ & $10^{-4}$ & $10^{-2}$ & $10^{-3}$ \\
$\mathrm{p}_{3 / 2}$ or $\mathrm{d}_{5 / 2}$ & 0.72 & $10^{-3}$ & 0.80 & $10^{-2}$ \\
$\mathrm{p}_{1 / 2}$ or $\mathrm{d}_{3 / 2}$ & 1.26 & 1.99 & 1.19 & 1.99 \\
\hline
\end{tabular}

situations. The $\mathrm{G}^{1}$ and $\mathrm{G}^{3}$ Slater-Condon parameters have an approximately constant ratio with respect to the $\mathrm{F}^{2}$ value. The important factor for the spectral shape is the ratio of the core spin-orbit coupling and the $\mathrm{F}^{2}$ value. Finite values of both the core spin-orbit and the Slater-Condon parameters cause the presence of the prepeak. It can be seen in Table 6 that the $3 p$ and $4 d$ spectra have small core spin-orbit couplings, implying small $p_{3 / 2}\left(d_{5 / 2}\right)$ edges and extremely small prepeak intensities. The deeper $2 p$ and $3 d$ core levels have larger core spin-orbit splitting with the result of a $p_{3 / 2}\left(d_{5 / 2}\right)$ edge of almost the same intensity as the $p_{1 / 2}\left(d_{3 / 2}\right)$ edge and a larger prepeak. Note that none of these systems comes close to the single-particle result of a 2:1 ratio of the $p$ edges or the $3: 2$ ratio of the $d$ edges. Figure 8 shows the X-ray

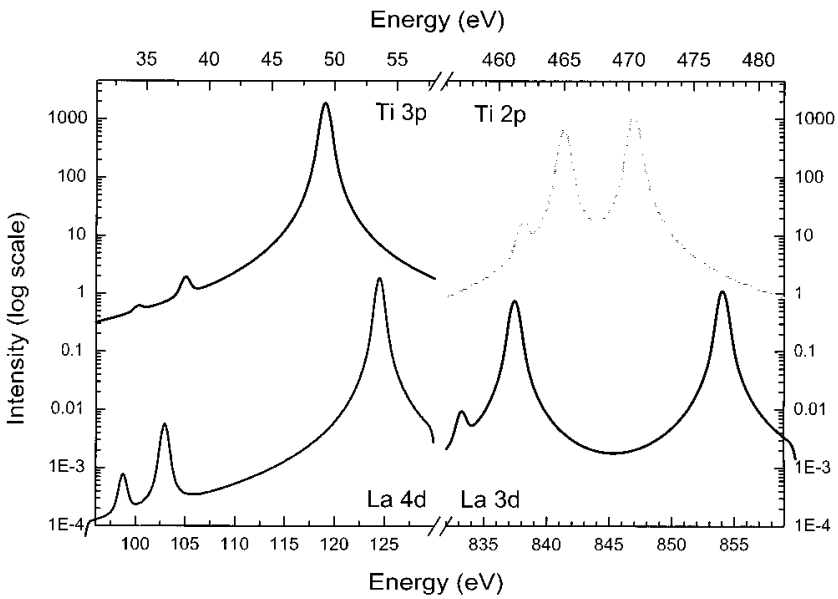

Figure 8. Atomic multiplet calculations for the $3 d^{0}$ and $4 f^{0}$ ions Tilv and Lalll. Given are the transitions $3 d^{0} \rightarrow$ $2 p^{5} 3 d^{1}, 3 d^{0} \rightarrow 3 p^{5} 3 d^{1}, 4 f^{0} \rightarrow 3 d^{9} 4 f^{1}$, and $4 f^{0} \rightarrow 4 d^{9} 4 f^{1}$. All spectra are given on a logarithmic scale to make the prepeaks clearly visible.

absorption spectral shapes. They are given on a logarithmic scale to make the preedges visible.

In Table 5 the term symbols of all $3 \mathrm{~d}^{\mathrm{N}}$ systems are given. Together with the dipole selection rules, this sets immediately strong limits to the number of final states which can be reached, similar to the case of a $3 d^{0}$ ground state. Consider, for example, the $3 d^{3} \rightarrow$ $2 p^{5} 3 d^{4}$ transition: The $3 d^{3}$ ground state has $\mathrm{J}=3 / 2$ and there are, respectively, 21, 35, and 39 states of $2 p^{5} 3 d^{4}$ with $J^{\prime}=1 / 2, J^{\prime}=3 / 2$, and $J^{\prime}=5 / 2$. This 
implies a total of 95 allowed peaks out of the 180 final-state term symbols. From Table 5 some special cases can be discriminated: a $3 d^{9}$ system makes a transition to a $2 \mathrm{p}^{5} 3 \mathrm{~d}^{10}$ configuration, which has only two term symbols, out of which only the term symbol with J ' $=3 / 2$ is allowed. In other words, the $L_{2}$ edge has zero intensity. $3 d^{0}$ and $3 d^{8}$ systems have only three and four peaks, respectively, because of the limited amount of states for the $2 p^{5} 3 d^{1}$ and $2 p^{5} 3 d^{9}$ configurations.

Atomic multiplet theory is able to accurately describe the $3 d$ and $4 d$ X-ray absorption spectra of the rare earth metals. ${ }^{55} \mathrm{In}$ the case of the $3 \mathrm{~d}$ metal ions, atomic multiplet theory cannot simulate the X-ray absorption spectra accurately because the effects of the neighbors on the $3 d$ states are too large. It turns out that it is necessary to include both the symmetry effects and the configuration-interaction effects of the neighbors explicitly. Ligand-field multiplet theory takes care of all symmetry effects, while chargetransfer multiplet theory allows the use of more than one configuration.

\section{B. Ligand-Field Multiplet Theory}

If one transfers this atomic method to the solid state, two questions that arise are (1) how to deal with the different local symmetry and (2) how to incorporate the different more itinerant electronic features of the solid state, such as the electron density of the s and p states. The inclusion of the local symmetry has been the subject of many studies under the heading of ligand-field theory. ${ }^{12}$ This has been used particularly to describe the optical absorption spectra of transition-metal ions. For core spectroscopies, ligand-field theory has been devel oped by Thole and co-workers. ${ }^{110}$ The effects of itinerant states on multiplets will be described using the charge-transfer multiplet approach as described in the next section.

The dominant symmetry effect in solids is the cubic ligand field. The strength of this operator is usually denoted as the ligand-field splitting (10Dq). Atomic multiplet theory can be extended to describe the $3 d$ metal ions by incorporation of the ligand-field splitting. In an octahedral environment, the field of the neighboring atoms on the of the central atom has cubic $\left(\mathrm{O}_{h}\right)$ symmetry which divides the 5-fold-degenerate $3 d$ orbitals into two distinct representations of $\mathrm{T}_{2 \mathrm{~g}}$ and $\mathrm{E}_{\mathrm{g}}$ symmetry. The 2-fold-degenerate $\mathrm{E}_{\mathrm{g}}$ state contains orbitals that point toward the center of the cube faces, which is directly toward the position of the ligands. Consequently, $\mathrm{E}_{\mathrm{g}}$ states interact stronger, electrostatically as well as covalently, with the ligands. The three $t_{2 g}$ orbitals point toward the corners of the cube, and therefore, their interaction with the octahedral ligands is smaller.

The effect of the cubic ligand field on the energies of the atomic states has been described in the textbooks of Ballhausen, ${ }^{51}$ Griffith, ${ }^{12}$ and Sugano, Tanabe, and Kitamura. ${ }^{105}$ It is found that the degeneracy of the atomic states is partially lifted and the D, F, and higher states are split into a series of representations in cubic symmetry. In the $2 \mathrm{p}^{5} 3 \mathrm{~d}^{\mathrm{N}}$ final state, the effects of the cubic ligand field are equivalent to that in the initial state. A difference is that the number of multiplet states is considerably larger: For a $3 d^{5}$ initial state, the maximum number of states is 256, while it is six times larger (1512) for a $2 p^{5} 3 d^{5}$ final state.

The transition probability for the $3 \mathrm{~d}^{0}$ systems in cubic symmetry is

$$
W \propto\left\langle 3 d^{0}\left[A_{1}\right]\left|p\left[T_{1}\right]\right| 2 p^{5} 3 d^{1}\left[T_{1}\right]\right\rangle^{2}
$$

All final states of $T_{1}$ symmetry are allowed and have a finite transition probability from the $A_{1}$ initial state. This includes the states of $\mathrm{J}=1$ atomic symmetry and additionally the atomic states with J $=3$ and J $=4$. The degeneracy of these states is, respectively, 3 and 1 . The total number of allowed final states in cubic symmetry is $3+3+1=7$. Figure 9 shows the

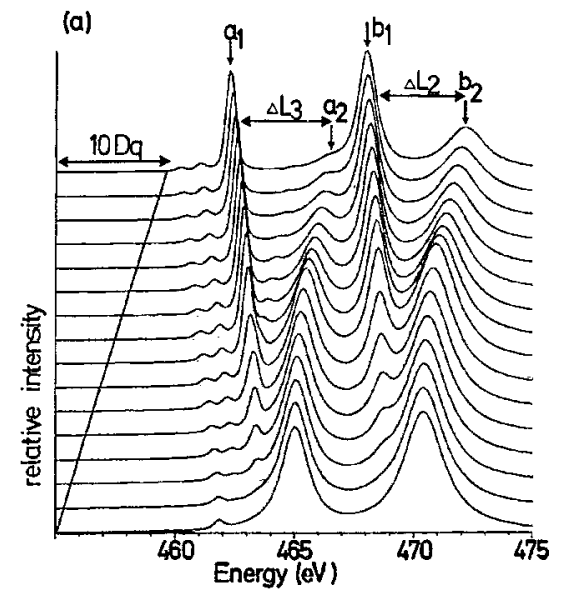

Figure 9. Ligand-field multiplet calculations of the transition $3 d^{0} \rightarrow 2 p^{5} 3 d^{1}$ for the Tilv system. The bottom spectrum has a cubic crystal field of $0.0 \mathrm{eV}$; the top spectrum has a cubic crystal-field value $10 \mathrm{Dq}$ of $4.5 \mathrm{eV}$. (Reprinted from ref 38. Copyright 1990 American Physical Society.)

effects of an increasing cubic ligand field on the multiplet spectrum of the $3 d^{0} \rightarrow 2 p^{5} 3 d^{1}$ transition. In this figure it can be seen that for small values of the ligand field (the step size is $0.3 \mathrm{eV}$ ) the spectrum is hardly modified. F or small values of $10 \mathrm{Dq}$, the four states which were forbidden in atomic symmetry have not gained enough intensity to be detectable in the spectrum. The peak splitting in the spectrum is related to the value of $10 \mathrm{Dq}$, but it is not a direct measure of it. This implies that one cannot measure the crystal-field splitting directly in a $2 p$ X-ray absorption spectra.

The ligand-field multiplet results as calculated with the procedure outlined above can be directly compared with experiment. Figure 10 compares the ligand-field multiplet calculation of $\mathrm{CaF}_{2}$ with the $2 \mathrm{p}$ $\mathrm{X}$-ray absorption spectrum of $\mathrm{CaF}_{2}$. Atomic Slater integrals were used. For details, see refs 37 and 38. All peaks in the experimental spectrum are reproduced, which is a confirmation that the ligand-field multiplets indeed dominate the spectral shape. When resonant X-ray emission will be discussed, $\mathrm{CaF}_{2}$ will be used as an example of what happens if one excites to these multiplet peaks. 


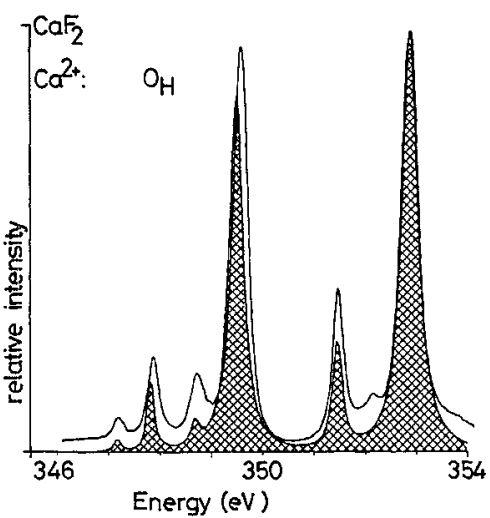

Figure 10. Calcium $2 p \mathrm{X}$-ray absorption spectrum of $\mathrm{CaF}_{2}$ compared with a ligand-field multiplet calculation. The value of $10 \mathrm{Dq}$ is $-0.9 \mathrm{eV}$. (Reprinted with permission from ref 38. Copyright 1990 American Physical Society.)

\section{Table 7: Projection of the $2 p$ X-ray Absorption Transitions from Atomic Symmetry $\left(\mathrm{SO}_{3}\right)$ to Octahedral Symmetry $\left(\mathrm{O}_{\mathrm{h}}\right)$. Degeneracies of the Configurations Are Given. The Last Column Gives the Degeneracies without Any Symmetry $\left(C_{1}\right)$}

\begin{tabular}{cccc}
\hline transition & $\begin{array}{c}\text { degeneracies } \\
\text { in spherical } \\
\text { symmetry }\end{array}$ & $\begin{array}{c}\text { degeneracies } \\
\text { in octahedral } \\
\text { symmetry }\end{array}$ & $\begin{array}{c}\text { degeneracies } \\
\text { without } \\
\text { symmetry }\end{array}$ \\
\hline $3 d^{0} \rightarrow 2 p^{5} 3 d^{1}$ & $1 \rightarrow 12$ & $1 \rightarrow 25$ & $1 \rightarrow 60$ \\
$3 d^{1} \rightarrow 2 p^{5} 3 d^{2}$ & $2 \rightarrow 45$ & $3 \rightarrow 90$ & $10 \rightarrow 270$ \\
$3 d^{2} \rightarrow 2 p^{5} 3 d^{3}$ & $9 \rightarrow 110$ & $20 \rightarrow 300$ & $45 \rightarrow 720$ \\
$3 d^{3} \rightarrow 2 p^{5} 3 d^{4}$ & $19 \rightarrow 180$ & $39 \rightarrow 420$ & $120 \rightarrow 1260$ \\
$3 d^{4} \rightarrow 2 p^{5} 3 d^{5}$ & $34 \rightarrow 205$ & $91 \rightarrow 630$ & $210 \rightarrow 1512$ \\
$3 d^{5} \rightarrow 2 p^{5} 3 d^{6}$ & $37 \rightarrow 180$ & $86 \rightarrow 420$ & $252 \rightarrow 1260$ \\
$3 d^{6} \rightarrow 2 p^{5} 3 d^{7}$ & $34 \rightarrow 110$ & $91 \rightarrow 300$ & $210 \rightarrow 720$ \\
$3 d^{7} \rightarrow 2 p^{5} 3 d^{8}$ & $19 \rightarrow 45$ & $39 \rightarrow 90$ & $120 \rightarrow 270$ \\
$3 d^{8} \rightarrow 2 p^{5} 3 d^{9}$ & $9 \rightarrow 12$ & $20 \rightarrow 25$ & $45 \rightarrow 60$ \\
$3 d^{9} \rightarrow 2 p^{5} 3 d^{10}$ & $2 \rightarrow 2$ & $3 \rightarrow 2$ & $10 \rightarrow 6$ \\
\hline
\end{tabular}

Table 7 gives the ground-state symmetries of the atomic multiplets and their projection to cubic symmetry. If four electrons have to be accommodated in the $3 \mathrm{~d}$ orbitals in an octahedral surrounding, two effects are important: The exchange coupling of two $3 d$ electrons $(J)$ and the cubic ligand-field splitting (D). The exchange coupling is connected to the Slater integrals: $\mathrm{J}=\left(\mathrm{F}^{2}+\mathrm{F}^{4}\right) / 14$, where the difference between the exchange couplings of $e_{g}$ and $t_{2 g}$ electrons is neglected. ${ }^{30}$ If $3 \mathrm{~J}>\mathrm{D}$, a high-spin configuration with ${ }^{5} \mathrm{E}$ symmetry is formed from the Hund's rule ${ }^{5} D$ ground state. If 3j $<\mathrm{D}$, a low-spin configuration with ${ }^{3} \mathrm{~T}_{1}$ symmetry is formed. It can be checked that the same criterion applies to a $3 d^{5}$ configuration, while for $3 d^{6}$ and $3 d^{7}$ configurations, one should compare 2) with D. The exchange coupling J is about $0.8 \mathrm{eV}$ for 3d electrons, implying a high-spin low-spin transition point of approximately $2.4 \mathrm{eV}$ for $3 d^{4}$ and $3 d^{5}$ and $1.8 \mathrm{eV}$ for $3 d^{6}$ and $3 d^{7}$.

The calculation of the ligand-field multiplet spectrum is equivalent to that of the $3 d^{0}$ configuration, with the additional possibility of low-spin ground states. To obtain the $2 p X$-ray absorption spectra, the calculated line spectrum is broadened with a Lorentzian broadening to simulate lifetime effects and a Gaussian broadening to simulate the resolution function of the experiment. Figure 11 shows the comparison for $\mathrm{MnO}$. Excellent agreement is obtained with the atomic values of the Slater integrals and a ligand-

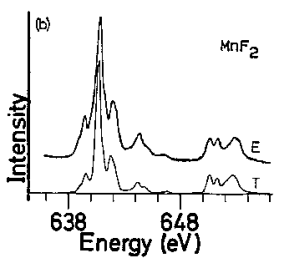

Figure 11. Manganese 2p X-ray absorption spectrum of $\mathrm{MnF}_{2}$ compared with ligand-field multiplet calculations. (Reprinted with permission from ref 37. Copyright 1990 American Physical Society.)

field splitting of $0.8 \mathrm{eV}$. Or, to be more specific, the Slater integrals have been calculated by HartreeFock, and these Hartree-Fock values have been reduced to $80 \%$ of their calculated value. This procedure reproduces the experimental Slater integrals of atomic spectra. In the case of covalent systems, further reduction of the Slater integrals (according to the nephel auxatic effect) is often used. If one uses the charge-transfer multiplet model, charge transfer should take care of the effective reduction of Slater integrals. F or more details, the reader is referred to refs 27 and 29 and references therein.

The transition from a high-spin to a low-spin ground state is directly visible in the spectral shape, because a different final-state multiplet is reached. This is shown theoretically and experimentally for a series of $\mathrm{Mn}^{11}$ compounds by Cramer and co-workers. ${ }^{26}$ An important advantage of soft X-ray edges is their high resolution, which can be less than $0.1 \mathrm{eV}$ due to the long lifetime of the core states. The high resolution makes the spectra sensitive to details of the electronic structure, such as the valence, symmetry, spin state, and crystal-field value.26,29 It is important to realize that multiplet effects are a general phenomenon in all cases where a $2 \mathrm{~s}, 2 \mathrm{p}$, or $3 p$ core hole is present in a $3 d$ metal.

\section{Charge-Transfer Multiplet Theory}

The essence of the charge-transfer model is the use of two or more configurations. Ligand-field multiplet calculations use one configuration for which it solves the effective atomic Hamiltonian plus the ligand-field Hamiltonian, so essentially the following matrixes

$$
\begin{gathered}
I_{X A S, 1} \propto\left\langle 3 d^{N}|p| 2 p^{5} 3 d^{N+1}\right\rangle^{2} \\
H_{I N T, 1}=\left\langle 3 d^{N}\left|\frac{e^{2}}{r_{12}}+\varsigma_{d} l_{d} \cdot S_{d}+H_{L F M}\right| 3 d^{N}\right\rangle \\
H_{F I N A L, 1}=\left\langle 2 p^{5} 3 d^{N+1}\right| \frac{e^{2}}{r_{12}}+\varsigma_{p} I_{p} \cdot S_{p}+\varsigma_{d} l_{d} \cdot S_{d}+ \\
H_{L F M}\left|2 p^{5} 3 d^{N+1}\right\rangle
\end{gathered}
$$

The charge-transfer model adds a configuration $3 d^{N+1} L$ to the $3 d^{N}$ ground state. In the case of a transition-metal oxide, in a $3 d^{N+1} \mathrm{~L}$ configuration an electron has been moved from the oxygen $2 p$ valence band to the metal $3 d$ band. The energy difference between the $3 \mathrm{~d}^{\mathrm{N}}$ and $3 \mathrm{~d}^{\mathrm{N}+1} \underline{\mathrm{L}}$ configurations is the so- 
called charge-transfer energy $(\Delta)$. One can continue with this procedure and add a $3 d^{N+2} \underline{L}^{2}$ configuration, etc. In many cases two configurations will be enough to explain the spectral shapes, but in particular for high valence states, it can be important to include more configurations. ${ }^{45,80}$ The $3 d$ states are described in a correlated fashion, while all other electrons are described in a band-like fashion. ${ }^{125}$ This is essentially the Anderson impurity model. The actual calculations can be carried out for a cluster or a quasiatomic model.

As far as X-ray absorption and X-ray emission are concerned, the consequences for the calculations are the replacement of $3 d^{N}$ with $3 d^{N}+3 d^{N+1} L$ plus the corresponding changes in the final state. This adds a second initial state, final state, and dipole transition

$$
\begin{aligned}
& \mathrm{I}_{X A S, 2} \propto\left\langle 3 d^{N+1} \underline{\underline{L}}|p| 2 p^{5} 3 d^{N+2} \underline{\underline{L}}\right\rangle^{2} \\
& H_{I N I T, 2}=\left\langle 3 d^{N+1} \underline{L}\left|\frac{e^{2}}{r_{12}}+\varsigma_{d} I_{d} \cdot S_{d}+H_{L F M}\right| 3 d^{N+1} \underline{L}\right\rangle \\
& H_{F I N A L, 2}=\left\langle 2 p^{5} 3 d^{N+2} \underline{L}\right| \frac{e^{2}}{r_{12}}+\varsigma_{p} I_{p} \cdot S_{p}+\varsigma_{d} l_{d} \cdot S_{d}+ \\
& H_{L F M}\left|2 p^{5} 3 d^{N+2} \underline{L}\right\rangle
\end{aligned}
$$

The two initial states and two final states are coupled by monopole transitions, i.e., configuration interaction. The mixing parameter $t$ couples both configurations, and as $\Delta$ is the energy difference, the Hamiltonian is indicated with $\mathrm{t} / \Delta$

$$
\begin{gathered}
H_{M I X \mid 1, I 12}=\left\langle 3 d^{N}|t / \Delta| 3 d^{N+1} \underline{L}\right\rangle \\
H_{M I X ~ F 1, F 2}=\left\langle 2 p^{5} 3 d^{N+1}|t / \Delta| 2 p^{5} 3 d^{N+2} \underline{L}\right\rangle
\end{gathered}
$$

The spectrum is calculated by solving the eqs $15 a-$ h. If a $3 d^{N+2} L L^{\prime}$ configuration is included, its energy is $2 \Delta+U$, where $U$ is the correlation energy between two 3d el ectrons. ${ }^{125}$ The formal definition of $U$ is the energy difference one obtains when an electron is transferred from one metal site to another, i.e., a transition $3 d^{N}+3 d^{N} \rightarrow 3 d^{N+1}+3 d^{N-1}$. The number of interactions of two $3 \mathrm{~d}^{\mathrm{N}}$ configurations is one more than the number of interactions of $3 \mathrm{~d}^{\mathrm{N}+1}$ plus $3 \mathrm{~d}^{\mathrm{N}-1}$, implying that this energy difference is equal to the correlation energy between two 3d electrons.

In the final state of the X-ray absorption process, the configurations are $2 p^{5} 3 d^{N+1}$ and $2 p^{5} 3 d^{N+2} L$. A $2 p$ electron is annihilated, and a $3 d$ electron is created. Because the $3 \mathrm{~d}$ el ectrons are relatively localized, this is an almost self-screened process. The consequence is that the relative ordering of the configurations does hardly change. To be precise: the energy difference between $2 p^{5} 3 d^{N+1}$ and $2 p^{5} 3 d^{N+2} L$ is given as $\Delta+U$ $-\mathrm{Q}$, where $\mathrm{U}$ is the $3 \mathrm{~d} 3 \mathrm{~d}$ correlation energy and Q the core hole potential, which identifies with the $2 p 3 d$ correlation energy. From the systematic analysis of core-level photoemission spectra, it has been found that $\mathrm{Q}$ is slightly larger than U. Typical values are $\mathrm{Q}$ equal to $9 \mathrm{eV}$ with $\mathrm{U}$ equal to 7 or $8 \mathrm{eV}$. This is the reason that only small charge-transfer satellites are visible in X-ray absorption, in contrast to the large satellites in photoemission. ${ }^{29}$

By analyzing the effects of charge transfer, it is found that (for systems with a positive value of $\Delta$ ) the main effects on the X-ray absorption spectral shape are (1) the contraction of the multiplet structures and (2) the formation of small satellites. Figure 12 shows the effect of the charge-transfer energy on

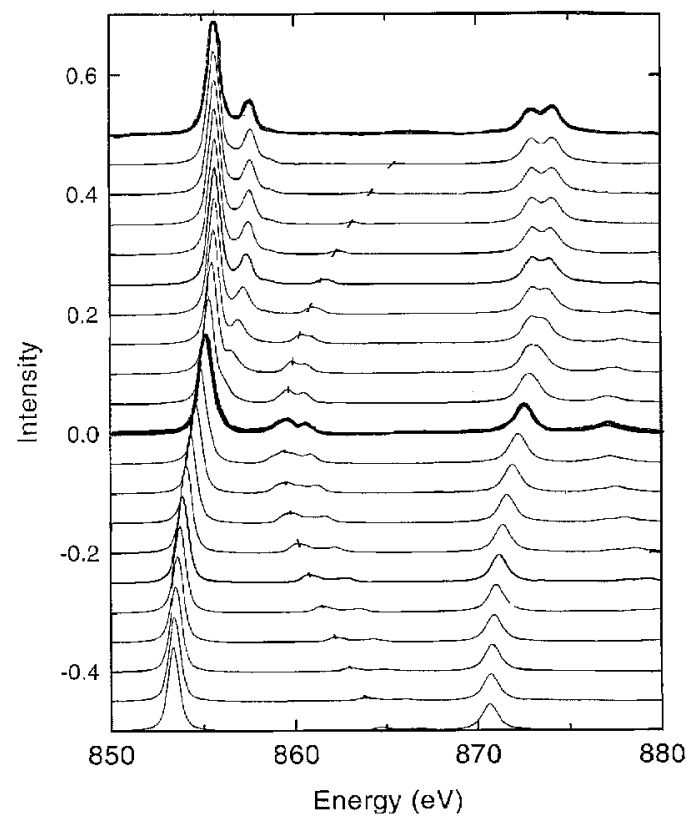

Figure 12. Series of chargetransfer multiplet calculations for the ground state $3 d^{8}+3 d^{9} L$. The top spectrum has a charge-transfer energy $\Delta$ of $+10 \mathrm{eV}$ and relates to an almost pure $3 d^{8}$ state; the bottom spectrum has a value of $\Delta$ of $-10 \mathrm{eV}$ and relates to an almost pure $3 \mathrm{~d}^{9} \mathrm{~L}$ state.

divalent nickel. In the top spectrum, $\Delta=10$ and the spectrum is essentially the ligand-field multiplet spectrum of a $\mathrm{Ni}^{11}$ ion in its $3 \mathrm{~d}^{8}$ configuration. The bottom spectrum uses $\Delta=-10$, and now the ground state is almost a pure $3 d^{9} \mathrm{~L}$ configuration. Looking for the trends in Figure $\overline{12}$, one nicely finds the increased contraction of the multiplet structure by going to lower values of $\Delta$. This is exactly what is observed in the series $\mathrm{NiF}_{2}$ to $\mathrm{NiCl}_{2}$ and $\mathrm{NiBr}_{2}{ }^{87-89,115}$ Going from $\mathrm{Ni}$ to $\mathrm{Cu}$, the atomic parameters change very little, except the $2 p$ spin-orbit coupling and the $2 p$ binding energy. Therefore, the spectra of $3 d^{N}$ systems of different elements are all very similar and the bottom spectrum is also similar to Cull systems. Therefore, one can also use the spectra with negative $\Delta$ values for $\mathrm{Cu}^{\prime \prime \prime}$ compounds, such as $\mathrm{La}_{2} \mathrm{Li}_{1 / 2} \mathrm{Cu}_{1 / 2} \mathrm{O}_{4}$ and $\mathrm{Cs}_{2} \mathrm{KCuF}_{6}$. Figure 13 shows the comparison of the $2 \mathrm{p}$ X-ray absorption spectrum of these two compounds with charge-transfer multiplet calculations. ${ }^{63,64}$ It can be checked in Figure 12 that these calculations look similar to the calculations for $\mathrm{Ni}{ }^{\prime \prime}$ systems with negative values of $\Delta$. For such systems with negative $\Delta$ values, it is important to carry out charge-transfer multiplet calculations as no good comparison with ligand-field multiplet spectra can be made. 


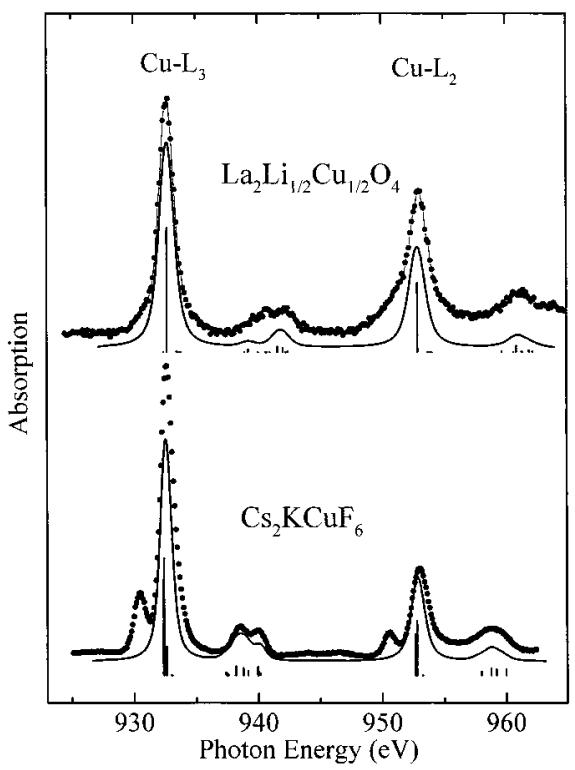

Figure 13. Results of theoretical simulations of the $\mathrm{Cu}$ $2 \mathrm{p}$ X-ray absorption spectra of $\mathrm{Cs}_{2} \mathrm{KCuF}_{6}$ (bottom) and $\mathrm{La}_{2} \mathrm{Li}_{1 / 2} \mathrm{Cu}_{1 / 2} \mathrm{O}_{4}$ (top), in comparison with the experimental spectra. (Reprinted with permission from ref 63. Copyright 1998 Elsevier Science.)

\section{1s X-ray Emission}

Now the X-ray emission spectra will be discussed. The theory for X-ray emission spectra and resonant $X$-ray emission spectra has been described in, for example, the works of Åberg ${ }^{1,2,113}$ and Kotani. ${ }^{81,106}$ In this paper the models derived in these works will be used, starting with a simpler model.

\section{A. 1s3p X-ray Emission in the Ligand-Field Multiplet Model}

First the 1s3p X-ray emission spectral shape will be discussed, also known as $\mathrm{K} \beta$ fluorescence. With divalent manganese as an example, a scheme to interpret the experimental spectral shapes using the multiplet models as described for X-ray absorption will be developed step by step. The ground state of $M n^{\prime \prime}$ has its five spin-up electrons filled. The five spin-down states are empty. It is first assumed that the core hole creation, for example, by an aboveresonance X-ray excitation, does not modify the valence electron situation. The intermediate state has a $1 s^{1} 3 d^{5}$ configuration and after the $1 s 3 p$ decay the final state is $3 p^{5} 3 d^{5}$

$$
3 d^{5} \rightarrow 1 s^{1} 3 d^{5}+\epsilon 1 s^{1} 3 d^{5} \Rightarrow 3 p^{5} 3 d^{5}
$$

The spectral shape consists of two structures separated by the 3p3d exchange interaction. This assignment is essentially the original model as used by Tsutsumi and co-workers. ${ }^{111,112}$ Starting from this model, the effects of the $3 p$ and $3 d$ spin-orbit coupling, the symmetry effects of the cubic crystal field, will step by step be included. In section V.A the model is extended to describe covalence effects as included with charge transfer and include the combined effects of off-resonance excitation and decay. Resonance effects will be discussed in section V.C.

Assuming a $\mathrm{Mn}^{11}$ ion, the atomic ground-state symmetry is ${ }^{6} \mathrm{~S}$. In the $1 \mathrm{~s}^{1} 3 \mathrm{~d}^{5}$ configuration, the total symmetry can be found by multiplying the 1s electron with the ${ }^{6} \mathrm{~S}$ symmetry of the $3 d$ electrons. This gives either a ${ }^{5} \mathrm{~S}$ state for antiparallel alignment of the $1 \mathrm{~s}$ and $3 \mathrm{~d}$ electrons or ${ }^{7} \mathrm{~S}$ for parallel alignment. The dipole selection rules imply that transitions are possible from ${ }^{5} S$ to ${ }^{5} \mathrm{P}$ and from ${ }^{7} \mathrm{~S}$ to ${ }^{7} \mathrm{P}$, with the energy difference between ${ }^{5} \mathrm{P}$ and ${ }^{7} \mathrm{P}$ given by the $3 p 3 d$ exchange interaction. Things are a bit more complex in reality because more then one ${ }^{5} \mathrm{P}$ configuration can be made from a $3 p^{5} 3 d^{5}$ configuration. Writing out all symmetry combinations one finds three ${ }^{5 \mathrm{P}}$ states and one ${ }^{7 \mathrm{P}}$ state. Figure 14a shows

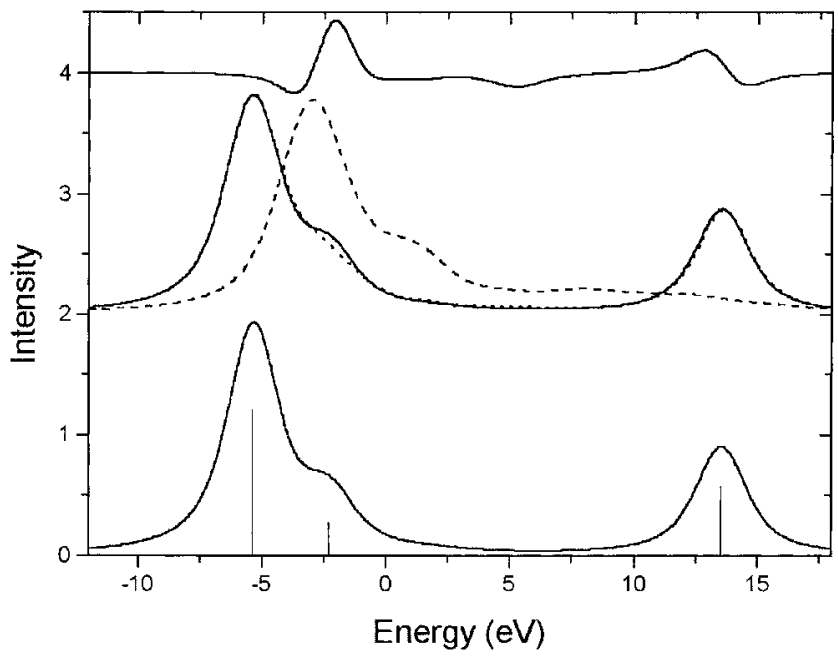

Figure 14. Theoretical $1 s 3 p$ X-ray emission spectral shapes calculated with the ligand-field multiplet model. Bottom: (a) The ligand-field, $3 d$ and $3 p$ spin-orbit couplings have all been set to zero. Middle: The spin-orbit couplings are set to their atomic values. (solid, b) A cubic crystal field of 2.0 has been added. (dotted, c) A cubic crystal field of $4.0 \mathrm{eV}$ has been added. (dashed, d) Top: Difference between $b$ and $c(\times 10)$.

the atomic multiplet spectrum for which the $3 d$ and $3 p$ spin-orbit couplings have been set to zero. The overall intensities of the states are 7:5 for the ${ }^{7} \mathrm{P}$ versus the ${ }^{5} \mathrm{P}$ states. The main peak is the ${ }^{7} \mathrm{P}$ state. The shoulder and the satellite are the ${ }^{5} \mathrm{P}$ states, and the small peak at about $-6482 \mathrm{eV}$ is the third ${ }^{5} \mathrm{P}$ state. The satellite can be related to the antiparallel aligned $3 p$ and $3 d$ electrons, while the shoulder and low-intensity state relate to orderings of the $3 d$ el ectrons different from the ground state. In fact, the three ${ }^{5} \mathrm{P}$ states are linear combinations of the three LS-like atomic states that are mixed by the strong $3 p 3 d$ as well as $3 d 3 d$ multiplet effects.

Figure $14 b$ shows the effects of the atomic $3 p$ and $3 d$ spin-orbit coupling. Essentially the ${ }^{7} P$ and ${ }^{5} P$ states are split into their three substates ${ }^{7} \mathrm{P}_{4},{ }^{7} \mathrm{P}_{3}$, and ${ }^{7} \mathrm{P}_{2}$. The intensities of the peaks are related to the J quantum numbers as $2 J+1$, and it can be seen that the lowest energy peak is the ${ }^{7} \mathrm{P}_{4}$ state. The situation for the ${ }^{5} \mathrm{P}$ states is similar, and the satellite is split into ${ }^{5} \mathrm{P}_{3},{ }^{5} \mathrm{P}_{2}$, and ${ }^{5} \mathrm{P}_{1}$, with the ${ }^{5} \mathrm{P}_{1}$ state at the lowest energy. A similar situation is visible for the shoulder at $-6487 \mathrm{eV}$. Comparing part $\mathrm{a}$ and $\mathrm{b}$ of Figure 14, it can be seen that the effects of the spin-orbit coupling on the broadened spectral shapes are minimal. 
Figure 14c shows the effects of the addition of a cubic crystal field of $2.0 \mathrm{eV}$. This is already a relatively large crystal field, and all oxides and halide systems have smaller crystal fields. It can be seen that compared to Figure 14b, most peaks shift a little bit. In particular, the ${ }^{5} \mathrm{P}$ states are modified while the ${ }^{7 P}$ states do still look very much like the atomic result. After broadening the sticks with the experimental and lifetime broadening, little changes are visible. This implies that $1 s 3 p$ X-ray emission spectra are insensitive to the crystal-field effects, much less sensitive than, for example, $2 p \mathrm{X}$-ray absorption. The positive aspect of this is that one can assume that the 1s3p X-ray emission spectra are virtually the same for all high-spin divalent manganese atoms. This finding is important for the use of the 1s3p X-ray emission channel for selective X-ray absorption measurements as will be discussed in section VII. It is noted that all other crystal-field effects in systems such as oxides will have no effect at all on the spectral shape (within the ligand-field multiplet model) as long as the spin state stays the same. Things are different for low-spin $\mathrm{Mn}^{\prime \prime}$ compounds. Figure 14d shows the effect of a cubic crystal field of $4.0 \mathrm{eV}$. A completely different spectrum is found. The reason is that the ground-state symmetry has changed from ${ }^{6} A_{1}$ to ${ }^{2} T_{2}$. Thus, only one unpaired $3 d$ electron remains, leading to a very small 3p3d exchange splitting and essentially no satellite. It is clear that 1s3p X-ray emission will immediately tell if a system is in its high-spin or low-spin state.

Similar to $2 p \mathrm{X}$-ray absorption, 1s3p X-ray emission is sensitive to the valence of the metal. $\mathrm{Mn}^{\prime \prime}$ has a $3 \mathrm{~d}^{5}$ ground state and $\mathrm{Mn}^{11 \prime}$ a $3 \mathrm{~d}^{4}$ ground state. ${ }^{90}$ This obviously leads to a different spectral shape in the experiments. Figure 15 shows an example of the

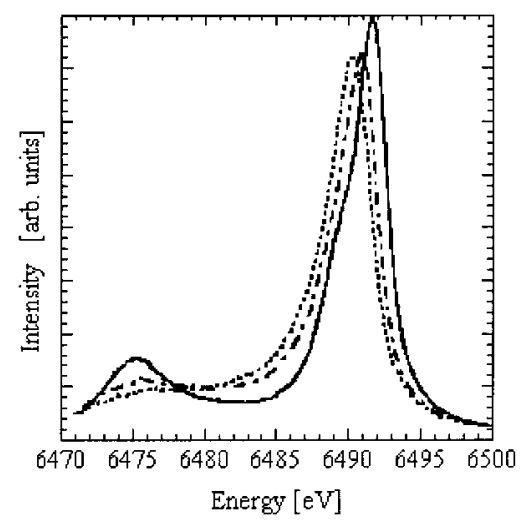

Figure 15. $1 s 3 p$ X-ray emission spectra of a $M n^{\prime \prime}(-)$, $M n^{\prime \prime \prime}(--)$ and $\mathrm{Mn}^{\prime V}(\cdot \cdots)$ compound. The compounds are, respectively, $\mathrm{Mn}(\mathrm{Oac})_{2}, \mathrm{Mn}(\mathrm{Oac})_{3}$, and $\mathrm{Mn}[\mathrm{HB}-$ $\left.\left(3,5-\mathrm{Me}_{2} \mathrm{pz}\right)_{3}\right]_{2}$. (Reprinted with permission from ref 90. Copyright 1994 American Chemical Society.)

variations in the manganese $1 s 3 p$ X-ray emission spectral shapes. Each spectrum can be simulated with a multiplet calculation leading to the determination of the valence. It can be seen that there is a clear shift between the spectra.

These chemical shifts have been studied in detail by Bergmann and co-workers, who established that it was more accurate to use the center of gravity of the 1s3p X-ray emission spectral shape as an energy indicator. 7,94 The nature of the $\mathrm{Mn}$ oxidation states involved in the oxygen-evolving complex in photosystem II has been studied with this method. The measurements have been made on the dark-adapted $\mathrm{S}_{1}$ state and on the hydroquinone-reduced state. The results are compatible with models involving conversion of $\mathrm{Mn}^{\mathrm{III}}{ }_{2} \mathrm{Mn}^{\mathrm{IV}}{ }_{2}$ to $\mathrm{Mn}_{2}{ }_{2} \mathrm{Mn}^{\mathrm{IV}}{ }_{2}$ clusters. ${ }^{7}$

\section{B. 1s3p X-ray Emission in the Charge-Transfer Multiplet Model}

In the previous sections the approximation that one can calculate the 1s3p X-ray emission spectral shape from a model using the following two transitions was used, where the excitation step is not used explicitly in the results

$$
3 d^{5} \rightarrow 1 s^{1} 3 d^{5}+\epsilon \mid s^{1} 3 d^{5} \Rightarrow 3 p^{5} 3 d^{5}
$$

In $\mathrm{Mn}^{\prime \prime}$, this model gives a good account of the spectral shape. If one looks at other systems though, an agreement that is not as good is found. The main reason for this is that in the 1s excitation step major screening or charge-transfer effects occur. Essentially one is removing a core electron, implying a core charge that has increased by one. This pulls down the valence $3 d$ electrons by the core hole potential. Because the $3 d$ el ectrons are pulled down in a larger energy than the $4 s$ and $4 p$ electrons, the intermediate-state configurations will be different from the ground-state configurations. Essentially one has to subtract the core hole potential Q from the groundstate energy positions as used in the charge-transfer model. Below the transitions are given for two configurations

$$
\begin{gathered}
3 d^{5}+3 d^{6} \underline{L} \rightarrow 1 s^{1} 3 d^{5} \epsilon+1 s^{1} 3 d^{6} \underline{L} \epsilon \\
1 s^{1} 3 d^{5}+1 s^{1} 3 d^{6} \underline{L} \text { (lowest) } \Rightarrow 3 p^{5} 3 d^{5}+3 p^{5} 3 d^{6} \underline{L}
\end{gathered}
$$

The lowest two intermediate states are selected, and the $1 s 3 p$ X-ray emission spectrum is calculated for these states, which implies that one assumed the intermediate states to relax to their lowest configuration(s). Two states are used, because the lowest state is split by the 1s3d exchange interaction. This 'relaxed' model has been used in the calculations of the 1s3p X-ray emission spectra of divalent nickel compounds. ${ }^{36}$ Using an intermediate-state configuration, $1 s^{1} 3 d^{8}+1 s^{1} 3 d^{9} \underline{L}+1 s^{1} 3 d^{10} L L^{\prime}$. The chargetransfer values for $\mathrm{NiB}_{2}$ and $\mathrm{NiF}_{2} \overline{\text { are }}$, respectively, 4.3 and $0.3 \mathrm{eV}$, and with a core hole potential of 7.5 $\mathrm{eV}$ and a correlation energy $\mathrm{U}$ of $7.3 \mathrm{eV}$, this gives that the lowest energy is the $1 s^{1} 3 d^{9}$ L configuration. $\mathrm{F}$ or $\mathrm{NiF}_{2}$, the $1 \mathrm{~s}^{1} 3 \mathrm{~d}^{8}$ configuration has an energy of $3.2 \mathrm{eV}$ and the $1 \mathrm{~s}^{13} \mathrm{~d}^{10} \mathrm{LL} \mathrm{L}^{\prime}$ configuration of $4.1 \mathrm{eV}$. In the case of $\mathrm{NiBr}_{2}$, these values are, respectively, $7.2 \mathrm{eV}$ and $0.1 \mathrm{eV}{ }^{36}$

The 'relaxed' charge-transfer model used is not necessarily the correct model. Another approach is to assume that the intermediate states do not relax at all. In this section this approximation will be 
followed. The model is the same as in the previous section

$$
\begin{gathered}
3 d^{5}+3 d^{6} \underline{\underline{L}} \rightarrow 1 s^{1} 3 d^{5} \epsilon+1 s^{1} 3 d^{6} \underline{\underline{L}} \epsilon \\
1 s^{1} 3 d^{5}+1 s^{1} 3 d^{6} \underline{L}(\text { XPS spectrum) }) \\
3 p^{5} 3 d^{5}+3 p^{5} 3 d^{6} \underline{L}
\end{gathered}
$$

One calculates the 1s XPS spectral shape, and then for all these intermediate states the 1s3p X-ray emission spectral shape is calculated. One then adds the 1s3p spectra with the intensities from the 1s XPS calculation. In principle, one should use the Kramers-Heisenberg formula to account for interference effects, but in the case of 1s core levels, interference effects are absent. In section $V$ the Kramers-Heisenberg formula for $2 \mathrm{p} 3 \mathrm{~s}$ and $2 \mathrm{p} 3 \mathrm{~d}$ resonant X-ray emission spectral shapes will be explicity used.

\section{K Capture 1s3p X-ray Emission}

The effects of the excitation process can be studied by using two different excitation processes. Recently Glatzel and co-workers compared the usual above resonance X-ray excitation with $\mathrm{K}$-capture excitation for $\mathrm{MnO} .{ }^{6}$ The X-ray excitation process follows the scheme as outlined above. The 1s electron leaves the atom as a continuum electron, and the spectrum is calculated by adding the 1s3p spectra of the 1s XPS spectrum states. In the K-capture process, the is electron annihilates a proton and is captured in the core. ${ }^{6}$ The calculation is the same as that for X-ray excitation as given above. The difference is the ordering of the configurations. As indicated in Figure 16 , the energy difference between $1 s^{1} 3 d^{5}$ and $1 s^{1} 3 d^{6} L$

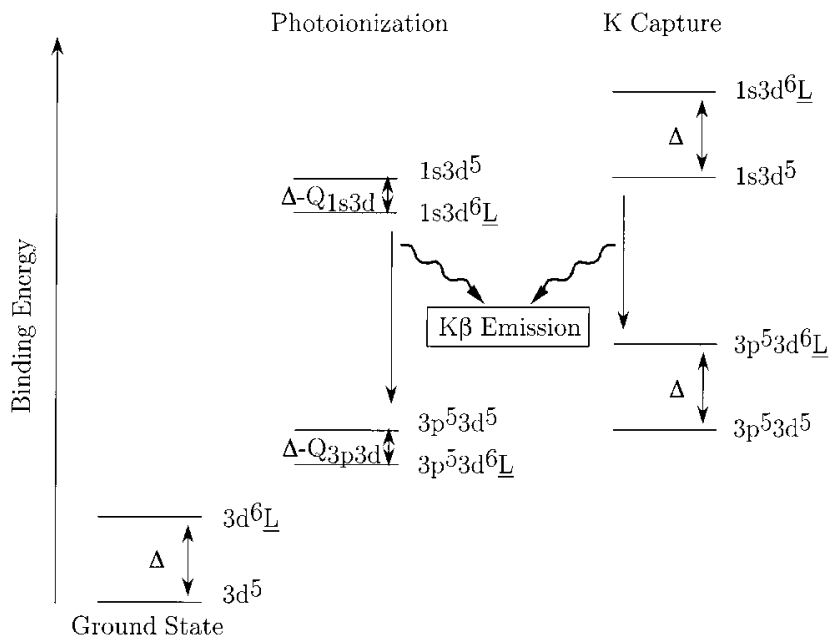

Figure 16. Scheme of the configurations in the initial, intermediate, and final states of, respectively, X-ray excited and $\mathrm{K}$-capture $\mathrm{X}$-ray emission calculations.

is equal to $\Delta-\mathrm{Q}$. Because the core hole potential is larger than the charge-transfer energy, this is a negative number and the lowest energy configuration is $1 s^{13} d^{6} L$. In the case of $K$ capture, the 1s electron that has 'left' is part of the nucleus and the total charge of the nucleus plus the 1s core level is not modified. This implies that the outer valence electrons hardly notice any effect on the positions of the various configurations and the energy difference between $1 s^{1} 3 d^{5}$ and $1 s^{1} 3 d^{6} L$ is equal to that in the ground state, being $\Delta$. The $\mathrm{K}$-capture spectrum is sharper, and the satellite structure is found at a lower energy, which is a consequence of additional states in the X-ray excited spectrum. The simulated spectra correspond nicely with the experimental results. ${ }^{6}$

\section{Temperature- and Pressure-Dependent 1s3p X-ray Emission}

1s3p X-ray emission spectra involve hard X-rays for both excitation and decay. Because only hard $X$-rays are involved in the experiment, one has a large flexibility in the experimental setup as well as sample environment. This makes it relatively straightforward to carry out the 1s3p X-ray emission experiment in situ, for example, at high-temperature, high-

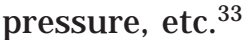

Tyson and co-workers ${ }^{91,114}$ measured a series of manganese perovskites using $1 s 3 p \mathrm{X}$-ray emission to determine the valences of $\mathrm{Mn}$ in these systems. In particular, they measured the temperature dependence of the 1s3p X-ray emission spectral shape. The low-temperature spectrum of $\mathrm{La}_{.5} \mathrm{Ca}_{.5} \mathrm{MnO}_{3}$ has more weight close to the center of gravity compared with the room temperature spectrum. From Figure 15 (and more clearly Figure 32b), it can be concluded that spectral weight closer to the center of gravity indicates a smaller 3p3d exchange splitting, implying less unpaired $3 d$ electrons. The reason for the decrease in unpaired $3 d$ electrons can be either a valence change or a spin transition. In the case of $\mathrm{La}_{5} \mathrm{Ca}_{5} \mathrm{MnO}_{3}$, the decrease of unpaired $3 d$-electrons is attributed to a valence increase. ${ }^{91}$ Rueff and coworkers ${ }^{97,98}$ used the 1s3p X-ray emission spectral shape for the analysis of pressure-dependent spectra of $\mathrm{Fe}$ and $\mathrm{FeS}$. The structural and magnetic transition between the magnetic (bcc) and the nonmagnetic (hcp) iron phases has been observed from the shift of intensity toward the center of gravity.

\section{C. $1 s 2 p$ X-ray Emission}

The situation of the $1 s 2 p$ or K $\alpha$ X-ray emission spectra is analogous to the 1s3p X-ray emission spectra. This situation is alike the comparison between $2 p$ X-ray absorption and 3p X-ray absorption. The calculation in the various models discussed above is exactly the same, the only difference being the different parameters of the $2 p$ instead of the $3 p$ core electrons. The $2 p$ spin-orbit coupling is much larger than the 3p spin-orbit coupling, typical values being, respectively, 10 and $1 \mathrm{eV}$. This implies that the spinorbit-split states are clearly visible in the experimental spectral shapes. In contrast, the 2p3d exchange interaction is much smaller than the $3 p 3 d$ exchange. This implies that the satellites visible in the $1 \mathrm{~s} 3 p$ $X$-ray emission spectra will only be shoulders in the case of $1 \mathrm{~s} 2 \mathrm{p} X$-ray emission.

Figure 17 shows the theoretical 1s2p X-ray emission spectra of $\mathrm{Fe}^{\mathrm{ll}}$, and Figure 18 shows the corresponding 1s3p X-ray emission spectra. The calculations are performed with the ligand-field multiplet approach as discussed in section IV.A. The spectra have been divided into spin-up and spin-down, and this aspect will be discussed in the next section. The 


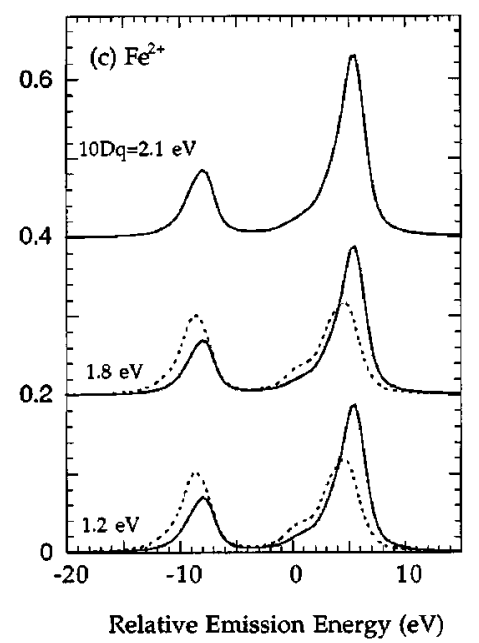

Figure 17. Theoretical spin-polarized 1s2p X-ray-emission spectra $\mathrm{Fe}^{\prime l}$ using the ligand-field multiplet calculation. Plotted are spin-down $(-)$ and spin-up spectra $(\cdot \cdots)$. (Reprinted with permission from ref 121. Copyright 1997 American Physical Society.)

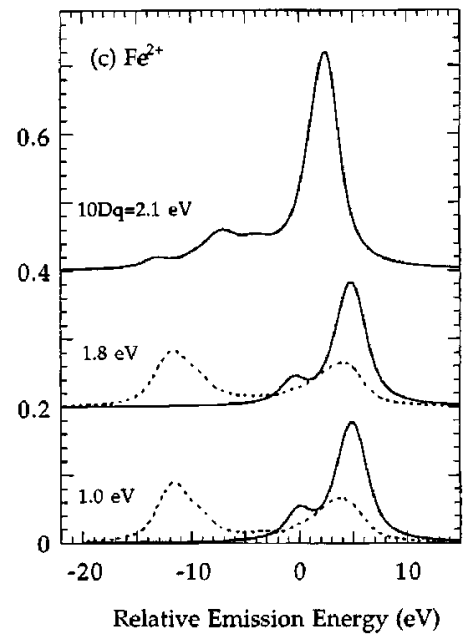

Figure 18. Theoretical spin-polarized 1s3p X-ray-emission spectra of $\mathrm{Fe}^{\mathrm{ll}}$ using the ligand-fiel d multiplet calculation. Plotted are spin-down $(-)$ and spin-up spectra ( $\cdots$ ). (Reprinted with permission from ref 121. Copyright 1997 American Physical Society.)

1s3p X-ray emission spectrum is split into a main peak at $+5 \mathrm{eV}$, where $0 \mathrm{eV}$ is the average $1 \mathrm{~s} 3 \mathrm{p}$ excitation energy. A satellite is visible at about -12 $\mathrm{eV}$, the energy difference being the 3p3d exchange splitting. The 1s $2 p \mathrm{X}$-ray emission spectrum also has its main peak at $+5 \mathrm{eV}$ and a satellite at about -9 $\mathrm{eV}$. This general spectral shape thus looks similar to the 1s3p case, but the origin of the splitting is different, $2 p$-spin-orbit, respectively, and 3p3d exchange. This assignment is confirmed if one looks at the top spectra that relate to low-spin $\mathrm{Fe}^{\mathrm{ll}}$ systems. Low-spin $\mathrm{Fe}^{\mathrm{ll}}$ has no unpaired $3 d$ electrons (i.e., a ${ }^{1} \mathrm{~A}_{1}$ ground state); hence, no exchange splitting and the satellite is absent. Only some low-energy shoulders are visible due to other (multiplet) effects. In the case of the $1 s 2 p$ X-ray emission spectrum, not much has changed for the low-spin situation. The $2 p$ spin-orbit coupling is the same, and the satellite maintains a similar structure and intensity.

The 1s2p X-ray emission spectrum is a few times more intense than the $1 s 3 p$ X-ray emission spectrum, and one might expect more studies using the $1 s 2 p$ channel. However, the chemical sensitivity of the 3p3d exchange interaction makes the 1s3p X-ray emission spectrum more useful for determining the spin state and valence. ${ }^{7,94}$

\section{1s2p Resonant X-ray Emission}

In this section I focus on the effects that occur if one excites at resonance. I will go deeper into this issue when the soft X-ray $2 p$ resonances, where interference effects are more important, are discussed. This section deals with resonance effects at the K edges. It will be shown that the study of $1 s 2 p$ resonant X-ray emission sheds light on the nature of the intermediate states, in other words the final states of the X-ray absorption process.

Figure 19 shows the $1 s 2 p$ resonant X-ray emission

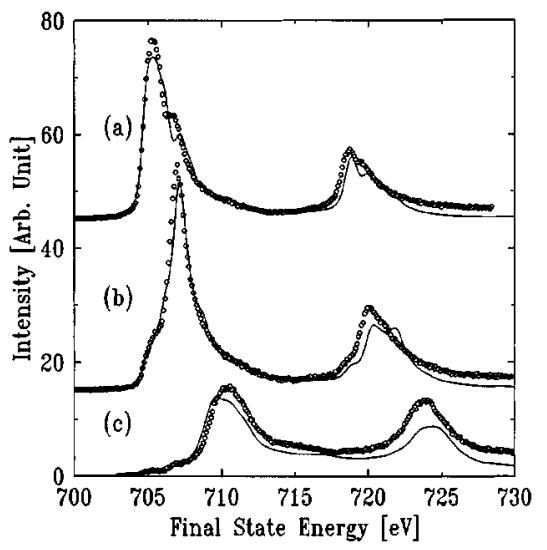

Figure 19. Calculated spectra (-) as a function of the energy difference of the incident and scattered photon together with the measured spectra (O). (Reprinted with permission from ref 23. Copyright 1998 American Physical Society.)

spectra of $\mathrm{Fe}_{2} \mathrm{O}_{3}$ excited at resonance. Spectra a, b, and $\mathrm{c}$ have been measured at the positions of the $\mathrm{K}$ edge as indicated in Figure 20. The spectrum is

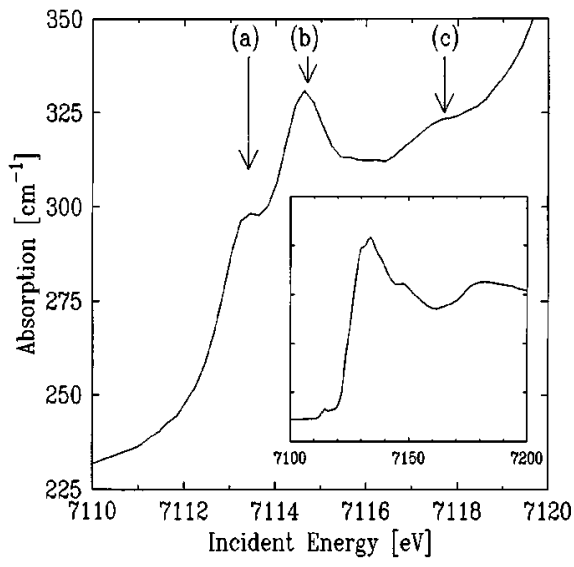

Figure 20. Fe K-absorption edge of $\mathrm{Fe}_{2} \mathrm{O}_{3}$. The preedge region is shown in more detail, and the whole edge is shown in the inset. (Reprinted with permission from ref 23. Copyright 1998 American Physical Society.)

normalized so that the measured absorption coefficients for photon energies immediately below and well above the absorption edge are the same as the theoretical values. Several weak preedge peaks are clearly observed. The first two preedge peaks, located 
at 7113.4 and $7114.8 \mathrm{eV}$, are assigned to the quadrupole transitions from the $\mathrm{Fe}$ 1s orbital to the crystal-field split $t_{2 g}$ and $e_{g}$ orbitals. These spectra have been measured by Caliebe and co-workers ${ }^{23}$ at NSLS beamline X21 with an overall resolution of 0.45 $\mathrm{eV}$. With this high experimental resolution, $1 \mathrm{~s} 2 \mathrm{p}$ X-ray emission spectra are measured well.

Recently, Dräger and co-workers performed similar experiments on single crystals of $\mathrm{MnO}$. The manganese 1s preedge structure is different for measurements with a (100) and (110) orientation of the rock salt crystal structure. The (100) spectrum gives transitions to the empty $e_{g}$ states and the (110) spectrum to the empty $t_{2 g}$ states. The resonant $1 s 3 p$ X-ray emission spectra have been used to disentangle the spin-polarization of the X-ray absorption spectrum. ${ }^{46}$

The $1 s 2 p$ resonant X-ray emission spectra can be calculated with the charge-transfer multiplet model as described for X-ray absorption above. Chargetransfer multiplet calculations have been carried out for the $3 d^{5}$ ground state, the $1 s^{1} 3 d^{6}$ intermediate states $\left(\phi_{x}\right)$, and the $2 p^{1} 3 d^{6}$ final states, including the quadrupole excitation (Q) and the dipole decay (D)

$$
\frac{\mathrm{d}^{2} \sigma}{\mathrm{d} \Omega \mathrm{d} \omega} \sim\left|\sum_{\phi_{\mathrm{x}}} \frac{\left\langle 2 \mathrm{p}^{5} 3 \mathrm{~d}^{6}|\mathrm{D}| 1 \mathrm{~s}^{1} 3 \mathrm{~d}^{6}\right\rangle\left\langle 1 \mathrm{~s}^{1} 3 \mathrm{~d}^{6}|\mathrm{Q}| 3 \mathrm{~d}^{5}\right\rangle}{\mathrm{E}_{1 \mathrm{~s}^{13} \mathrm{~d}^{6}}-\mathrm{E}_{3 \mathrm{~d}^{5}}-\hbar \omega-\mathrm{i} \Gamma_{1 \mathrm{~s}}}\right|^{2}
$$

Three configurations $3 d^{5}, 3 d^{6} \underline{L}$, and $3 d^{7} L L^{\prime}$ ' have been used for the ground state, and the corresponding three configurations have been used for the intermediate and final states. The result of the calculations is indicated in Figure 19 as the solid lines. For these calculations, the dipole transitions to $1 s^{1} 3 d^{5} 4 p^{1}$ states have been included also but they do not significantly affect the calculated spectral shapes. ${ }^{23}$

Why is this resonant experiment so interesting? One could say that the only result of the experiment is a $1 s 2 p$ resonant $X$-ray emission spectrum that could also be measured directly with $2 p$ X-ray absorption. There are at least two interesting consequences of this experiment. First, it adds additional proof as to the quadrupole nature of the prepeaks in the $\mathrm{K}$ edge of $\mathrm{Fe}_{2} \mathrm{O}_{3}$. Second, it makes it possible to measure a spectrum similar to $2 p \mathrm{X}$-ray absorption by making use of hard $X$-rays. This makes it possible to measure these spectra under essentially any condition and as such gives a good possibility for in situ measurements in, for example, el ectrochemistry or catalysis research.

\section{E. 1s Valence Band X-ray Emission}

Valence-to-core transitions are obvious candidates for chemically sensitive X-ray emission spectra, since the character of the valence orbitals changes the most between different chemical species. Figure 21 sketches the various fluorescence transitions to a is core hole in $\mathrm{MnO}$. The binding energy of the manganese 1s core hole is approximately $6540 \mathrm{eV}$. Dipole transitions are possible from the manganese $2 p, 3 p$, and $4 p$ levels. This gives, respectively, $1 \mathrm{~s} 2 \mathrm{p}(\mathrm{K} \alpha)$, $1 \mathrm{~s} 3 \mathrm{p}\left(\mathrm{K} \beta_{1,3}\right)$, and 1s valence band $\left(\mathrm{K} \beta_{2,5}\right)$ X-ray emission. ${ }^{83}$ The manganese $4 p$ states are hybridized with the oxygen $2 s$ and $2 p$ states. This induces $X$-ray emission peaks at energies related to the oxygen $2 s$ and $2 p$ states. The

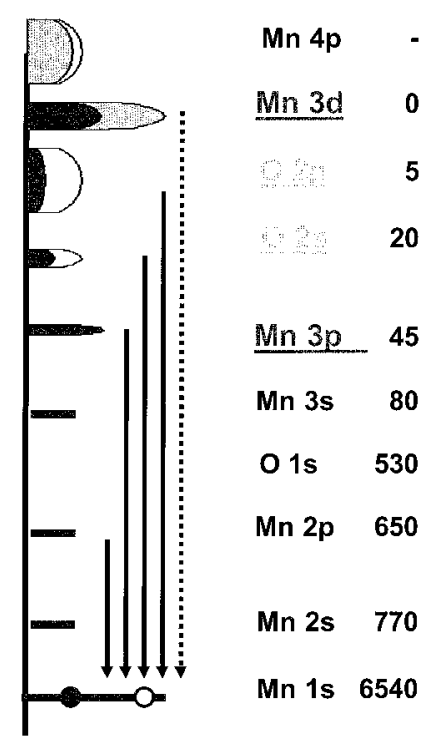

Figure 21. X-ray emission decay to a 1s core hole in $\mathrm{MnO}$. Indicated are, respectively, $1 \mathrm{~s} 2 \mathrm{p}(\mathrm{K} \alpha), 1 \mathrm{~s} 3 \mathrm{p}\left(\mathrm{K} \beta_{1,3}\right)$, and 1svalence band $\left(\mathrm{K} \beta_{2,5}\right) \mathrm{X}$-ray emission.

peak related to the $2 \mathrm{~s}$ state is sometimes referred to as crossover $\mathrm{X}$-ray emission. The peaks related to the oxygen $2 p$ bands are the valence band $X$-ray emission spectra. Apart from dipole X-ray emission, there can be quadrupole X-ray emission directly from the $3 d$ states.

Figure 22 shows a comparison of a $\mathrm{K} \beta$, crossover,

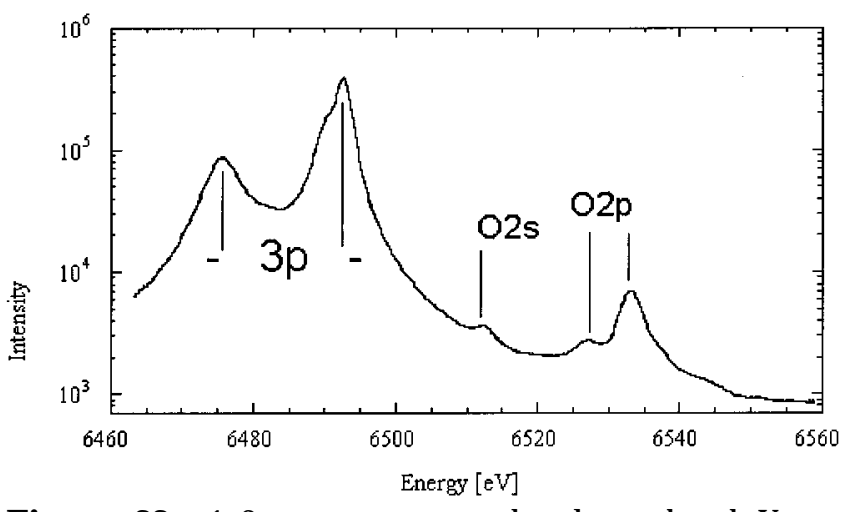

Figure 22. $1 s 3 p$, crossover, and valence band X-ray emission spectrum of $\mathrm{MnO}$. The intensity is given on a logarithmic scale.

and valence band X-ray emission spectra of $\mathrm{MnO}{ }^{8}$ As indicated in the figure, there is a $\mathrm{K} \beta$ peak related to manganese $3 p$ core holes, the crossover peak related to oxygen $2 \mathrm{~s}$ core holes, and the valence band realted to oxygen $2 p$ holes. Figure 23 shows a series of valence band X-ray emission spectra for manganese oxides. The bottom two spectra are manganese metal and $\mathrm{MnF}_{2}$. The energy of the valence band peak in the oxides shifts approximately $1 \mathrm{eV}$ per increment in oxidation state. This is indicated in the inset. $^{8}$

The intensities of the crossover peaks change significantly in the various oxides indicated in Figure 24. The integrated intensity of the crossover peak can be normalized by the main $\mathrm{K} \beta$ region, correcting for the number of oxygen atoms per $\mathrm{Mn}$. Assuming that the integrated intensity per $\mathrm{Mn}$ of the main $\mathrm{K} \beta$ region 


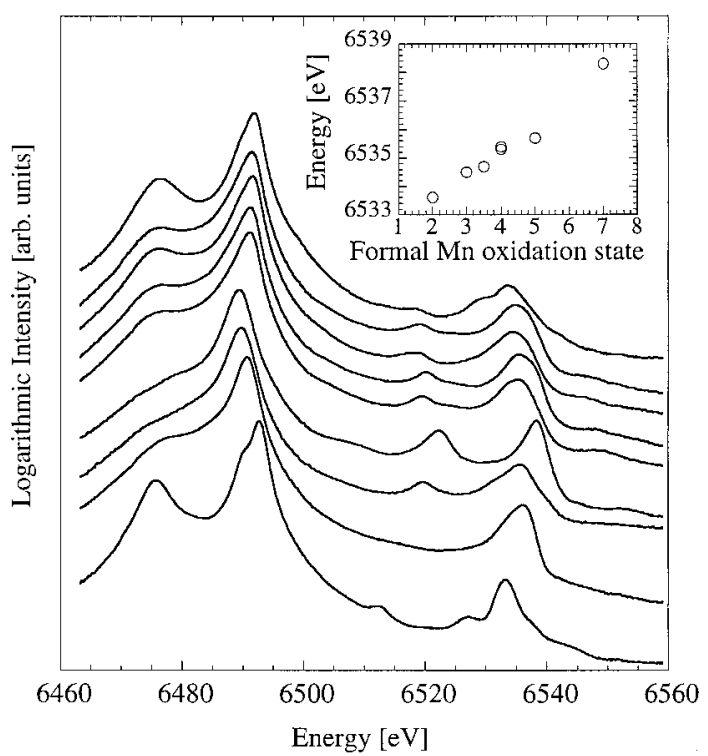

Figure 23. Logarithmic intensity plot of $\mathrm{Kb}$ spectra for a variety of $\mathrm{Mn}$ complexes: (top to bottom) $\mathrm{MnO}, \mathrm{LiMn}_{2} \mathrm{O}_{4}$, $\mathrm{ZnMn}_{2} \mathrm{O}_{4}, \mathrm{Li}_{2} \mathrm{MnO}_{3}, \mathrm{MnO}_{2}, \mathrm{KMnO}_{4}, \mathrm{~A} \mathrm{Mn}$ (IV) complex, $\mathrm{Mn}$ metal, and $\mathrm{MnF}_{2}$. (inset) $\mathrm{K} \beta_{2,5}$ main peak energies as a function of $\mathrm{Mn}$ formal oxidation state. (Reprinted with permission from ref 8. Copyright 1999 Elsevier Science.)

is chemically invariant, this procedure yields the relative crossover transition probability per $\mathrm{Mn}-\mathrm{O}$ pair. The inset of Figure 24 shows that the bond length varies exponentially with distance, and it can be concluded that the intensity of the crossover peak can be used as a tool to determine $\mathrm{Mn}-\mathrm{O}$ distances to within $0.1 \AA{ }^{8}$

\section{V. $2 p$ X-ray Emission}

The $2 p$ core levels of the $3 d$ transition metals are positioned in the soft X-ray range between 400 and $1000 \mathrm{eV}$. As discussed in section III, the $2 \mathrm{p}$ X-ray absorption spectra are dominated by multiplet effects and do show a complex multipeaked spectral shape. This makes $2 p$ X-ray emission ideally suitable for resonance studies. Therefore, this begins with a discussion of the resonant $2 p \mathrm{X}$-ray emission spectral shapes. Both 2p3s and 2p3d resonant X-ray emission will be discussed.

If the core hole is created with an energy close to the $2 p$ absorption edge, the X-ray absorption and $X$-ray emission processes do occur coherently and the overall $2 \mathrm{p} 3 \mathrm{~d}$ process (of a $3 d^{5}$ ground state) is described with the Kramers-Heisenberg formula

$$
I\left(\omega, \omega^{\prime}\right) \sim\left|\sum_{2 p^{5} 3 d^{6}} \frac{\left\langle 3 d^{5}\left|\hat{e}^{\prime} \cdot r\right| 2 p^{5} 3 d^{6}\right\rangle\left\langle 2 p^{5} 3 d^{6}|\hat{e} r| 3 d^{5}\right\rangle}{E_{2 p^{5} 3 d^{6}}-E_{3 d^{5}}-\hbar \omega-i \Gamma_{2 p}}\right|_{2}
$$

This formula forms the basis of all resonant X-ray processes. The intensity $(I)$ is given as a function of the excitation $(\omega)$ and the emission $\left(\omega^{\prime}\right)$ energies. The initial state $\left(3 d^{5}\right)$ is excited to an intermediate state $\left(2 p^{5} 3 d^{6}\right)$ with the dipole operator (êr), and the second dipole operator $\left(\hat{e}^{\prime} \cdot r\right)$ describes the decay to the final state $\left(3 d^{5}\right)$. The denominator contains the binding energy of the intermediate state and its lifetime

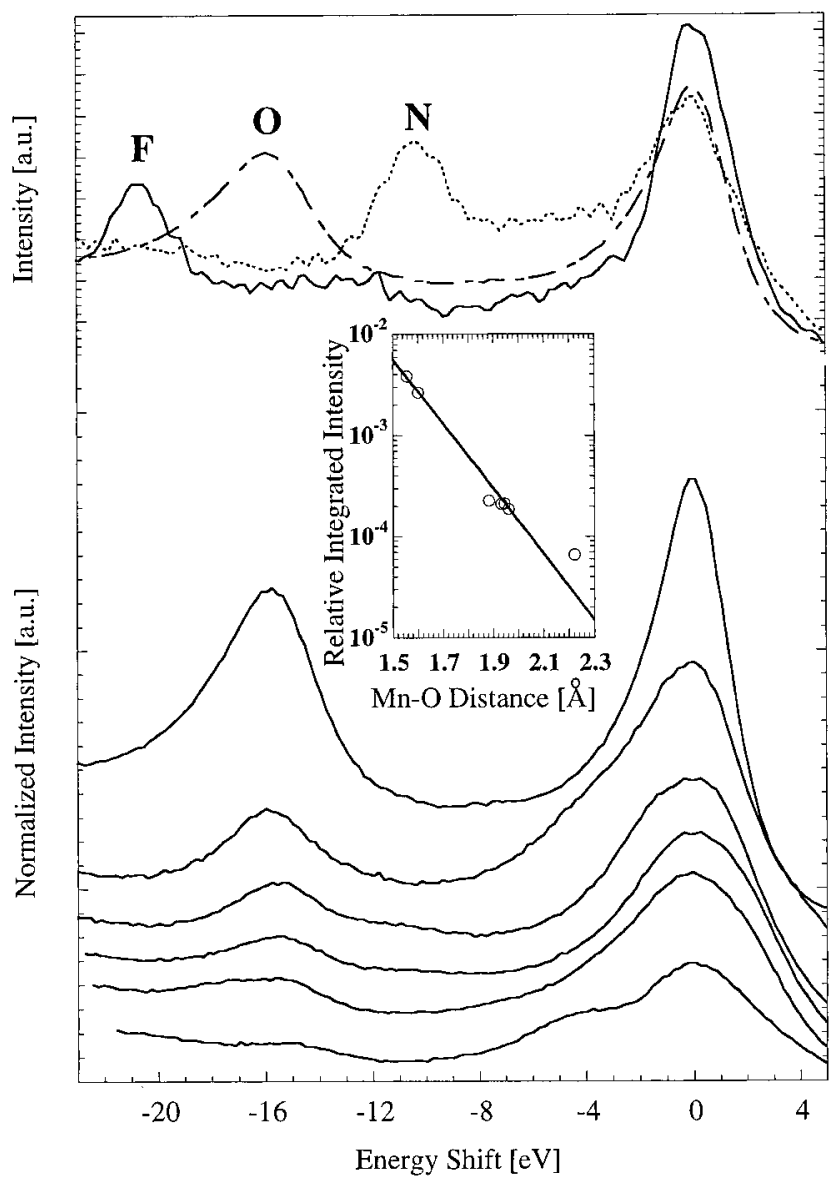

Figure 24. (top) $\mathrm{K} \beta^{\prime \prime}$ and $\mathrm{K} \beta_{2,5}$ regions for samples with $\mathrm{Mn}-\mathrm{F}, \mathrm{Mn}-\mathrm{O}$, and $\mathrm{Mn}-\mathrm{N}$ ligation. (bottom) $\mathrm{K} \beta^{\prime \prime}$ and $\mathrm{K} \beta_{2,5}$ regions of manganese oxides with different $\mathrm{Mn}$ oxidation states. (top to bottom) $\mathrm{KMnVII}_{4},\left[\mathrm{Et}_{4} \mathrm{~N}\right]\left[\mathrm{MnV}(\mathrm{O})\left(\eta^{4}-\mathrm{L}\right)\right]$, $\beta-\mathrm{Mn}^{\prime V} \mathrm{O}_{2}, \mathrm{LiMn}^{\prime \prime \prime} \mathrm{Mn}^{\mathrm{IV}} \mathrm{O}_{4}$ and $\mathrm{ZnMn}{ }^{\prime \prime \prime}{ }_{2} \mathrm{O}_{4}, \mathrm{Mn}^{\prime \prime} \mathrm{O}, \mathrm{Li}_{2^{-}}$

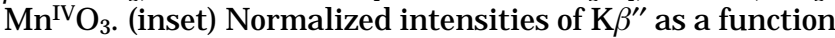
of $\mathrm{Mn}-\mathrm{O}$ distance. (Reprinted with permission from ref 8. Copyright 1999 Elsevier Science.)

broadening. A resonance occurs if the excitation energy is equal to the binding energy of the intermediate state. Looking at the MnO 2p X-ray absorption spectrum of Figure 11, each multiplet line can give rise to a different resonant $2 \mathrm{p} 3 \mathrm{~d} \mathrm{X}$-ray emission spectral shape.

The general spectral landscape can be viewed as a two-dimensional space with axis $\omega$ and $\omega^{\prime}$. Figure 26 shows a contour plot of the $2 p 3 d$ resonant X-ray emission spectrum of $\mathrm{Ni}^{\prime \prime}$ using a crystal field of 1 . $12 \mathrm{eV}$ to simulate NiO. The darkest area has the highest intensity. The horizontal axis shows the $X$-ray excitation energy. The main peak of the $2 p$ $\mathrm{X}$-ray absorption spectrum of $\mathrm{NiO}$ has an energy of $855.7 \mathrm{eV}$ with a shoulder at $857.5 \mathrm{eV} .{ }^{50}$ The vertical axis shows the $\mathrm{X}$-ray energy loss, that is the energy of $0.0 \mathrm{eV}$ means that the $2 \mathrm{p} 3 \mathrm{~d}$ decay energy is equal to the $2 p 3 d$ excitation energy, in other words this refers to resonant elastic scattering. There is a large peak visible with its peak position at $(855.7 \mathrm{eV}, 0.0$ $\mathrm{eV}$ ). There is a second peak at an energy loss of 1.1 $\mathrm{eV}$. This peak relates to a ligand-field excitation from the ${ }^{3} A_{2}$ ground state to the ${ }^{3} T_{2}$ excited state. $A$ third peak is visible at approximately $-2.0 \mathrm{eV}$.

A number of cross sections can be made. This paper focuses on the (resonant) X-ray emission spectral 
shape excited with a fixed energy. This will be indicated as $I^{\omega} \operatorname{XES}\left(\omega^{\prime}\right)$. In Figure 25 , this relates to

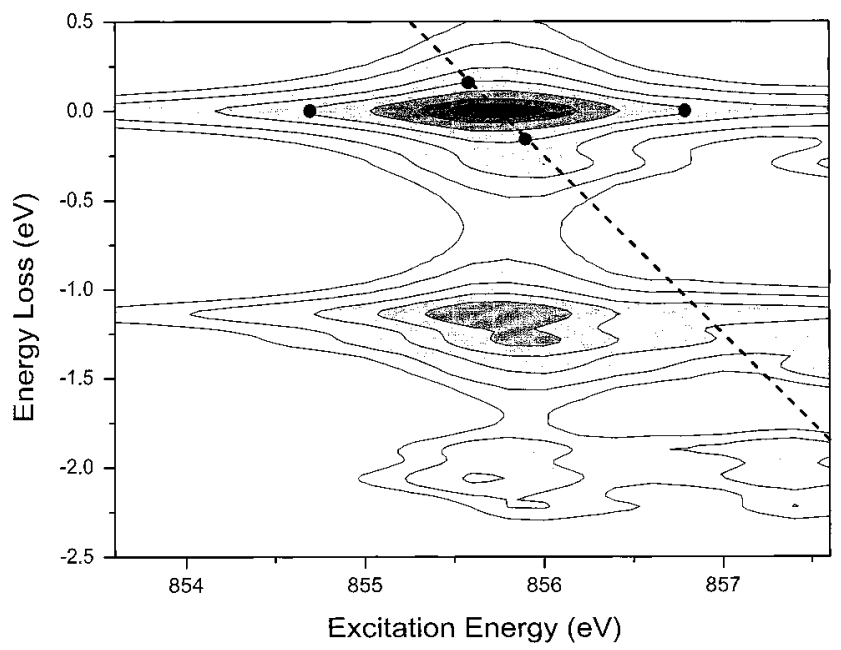

Figure 25. Contour plot of the resonant X-ray emission channels of a $\mathrm{Nill}$ ion with its ligand field set at $1.12 \mathrm{eV}$ to simulate $\mathrm{NiO}$. The excitation energy is given on the horizontal axis; the final state energy loss is shown on the vertical axis.

vertical cross sections. For example, the resonant $\mathrm{X}$-ray emission spectrum excited at $855.7 \mathrm{eV}$ relates to a vertical line at $855.7 \mathrm{eV}$. If the excitation energy is (far) above a resonance, this is normal X-ray emission or fluorescence. Close to a resonance, it is called resonant X-ray emission. With 2 p3d resonant $\mathrm{X}$-ray emission, one studies the elementary excitations by probing the energy difference between the initial and final state. These spectra can be plotted in two ways: (1) as resonant X-ray emission spectra, i.e., I ${ }^{\omega}$ XES $\left(\omega^{\prime}\right)$, or (2) as resonant inelastic X-ray scattering I ${ }^{\omega}$ XEs $\left(\omega^{\prime}-\omega\right)$. These spectra are off course exactly the same, but if one compares spectra excited at different excitation energies, the resonant X-ray emission spectra shift with the excitation energies while the RIXS spectra align all elementary excitations. RIXS can be used to study, for example, the dd excitations in transition metals as will be discussed below. ${ }^{41}$

Another cross section is to measure the X-ray absorption spectrum at a fixed emission energy: $I^{\omega^{\prime}} \mathrm{XAS}(\omega)$. This relates to the diagonal line in Figure 25. It is this mode that is used in selective XAFS, discussed in section VII. From Figure 25 one can derive the information on the effective lifetime re lated broadening. The $2 p$ intermediate state lifetime broadening has been made artificially large and the full-width half-maximum is about $2.2 \mathrm{eV}$, indicated by the distance between the two dots. The lifetime broadening of the $3 \mathrm{~d}$ final state has been set to fwhm $=0.32 \mathrm{eV}$. If one measures al ong the diagonal I ${ }^{\omega}{ }^{\prime} \mathrm{XAS}-$ $(\omega)$, the two points where the line crosses the halfmaximum point are indicated with dots. Reading of the difference in excitation energy one finds about $0.3 \mathrm{eV}$. This is a graphical illustration of the 'disappearance' of the intermediate-state lifetime broadening that will be discussed in more detail in section VII. The effective lifetime broadening of selective $\mathrm{X}$-ray absorption (for a single peak) is equal to
$1 / \sqrt{ }\left[\left(1 / \Gamma_{2 \mathrm{p}}^{2}\right)+\left(1 / \Gamma_{3 \mathrm{~d}}^{2}\right)\right], 32$ which for the present case gives $0.29 \mathrm{eV}$. It is interesting to compare this with off-resonance $\mathrm{X}$-ray emission where the effective lifetime broadening equals $\Gamma_{2 p}+\Gamma_{3 d}$, which is 2.52 $\mathrm{eV}$ in the present case.

\section{A. 2p3s Resonant X-ray Emission}

Before returning to the $2 \mathrm{p} 3 \mathrm{~d}$ resonant $\mathrm{X}$-ray emission spectra, the 2p3s resonant X-ray emission spectra will be discussed first as the 2 p3s case gives a good opportunity to describe essentially all peaks with a simple model. The calculation of the 2p3s resonant X-ray emission spectra of $\mathrm{CaF}_{2}$ will be followed. The X-ray absorption spectrum of $\mathrm{CaF}_{2}$ has been given in Figure 10 . In resonant 2p3s X-ray emission, one is exciting at all seven peaks in the absorption spectrum and measures the 2p3s X-ray emission spectral shape, as measured by Rubensson and co-workers. ${ }^{95,96}$ The 3s2p inelastic X-ray scattering cross section is dominated by resonant scattering via the $2 p^{5} 3 d^{1}$ intermediate states. It has been shown that one can reduce the calculation to the transitions of $3 d^{0}$, via $2 p^{5} 3 d^{1}$, to $3 s^{1} 3 d^{1}$ states. The effects of charge transfer are small and can be neglected. Both the X-ray absorption and X-ray emission steps essentially conserve the local charge, and it has been shown that in those experimental conditions, ionic systems such as $\mathrm{CaF}_{2}$ can be described in cl ose detail by the ligand-field approximation. One has to fill in the X-ray absorption and X-ray emission steps in the Kramers-Heisenberg formula

$$
\begin{aligned}
& \text { I }\left(\omega, \omega^{\prime}\right) \sim \\
& \left|\sum_{2 p^{5} 3 d^{1}} \frac{\left\langle 3 s^{1} 3 d^{1}\left|\hat{e}^{\prime} \cdot r\right| 2 p^{5} 3 d^{1}\right\rangle\left\langle 2 p^{5} 3 d^{1}|\hat{e} r| 3 d^{0}\right\rangle}{E_{2 p 53 d^{1}}-E_{3 d 0}-\hbar \omega-i \Gamma_{2 p}}\right|
\end{aligned}
$$

This calculation can almost be carried out 'by hand'. Starting from the situation in $\mathrm{CaF}_{2}$ with all bands completely full, one has the advantage of strong restrictions on the possible symmetry states. Ligandfield multiplet calculations have been performed for the $3 d^{0}$ initial state, the $2 p^{5} 3 d^{1}$ intermediate states, and the $3 s^{1} 3 d^{1}$ final states in cubic symmetry. The $3 d^{0}$ initial state consists of only one state with ${ }^{1} A_{1}$ symmetry. The only $2 \mathrm{p}^{5} 3 \mathrm{~d}^{1}$ intermediate states thatcan be reached in X-ray absorption must have $\mathrm{T}_{1}$ symmetry, and as there will be seven of those states. ${ }^{38}$ The $3 s^{1} 3 d^{1}$ final states are split by only two interactions, the 3s3d exchange and the cubic crystal field. This gives a total of four peaks in the final state. The complete calculation is then the transition of one initial state via seven intermediate states to four final states. The results of these seven absorption matrix elements and 28 (in fact 49 due to degeneracies) ${ }^{31}$ emission matrix el ements are fed into the KramersHeisenberg formula and this gives Figure 26 as its result.

Figure 27 gives the resulting resonant 2p3s X-ray emission spectral shapes. One can observe that in all comparisons of interference-on (solid line) and interference-off (dashed), the integrated intensities of the solid and dashed line are equivalent or in fact identical as the integrated intensity is conserved by 


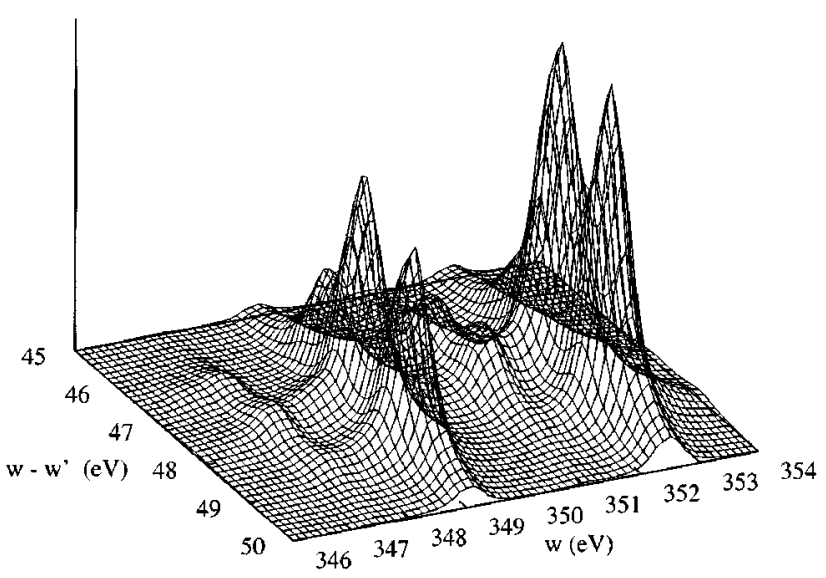

Figure 26. Plot of the two-dimensional resonant landscape of $\mathrm{CaF}_{2}$. The excitation energy is given on the $x$ axis; the final-state energy is given on the y axis. (Reprinted from ref 31. Copyright 1996 American Physical Society.)

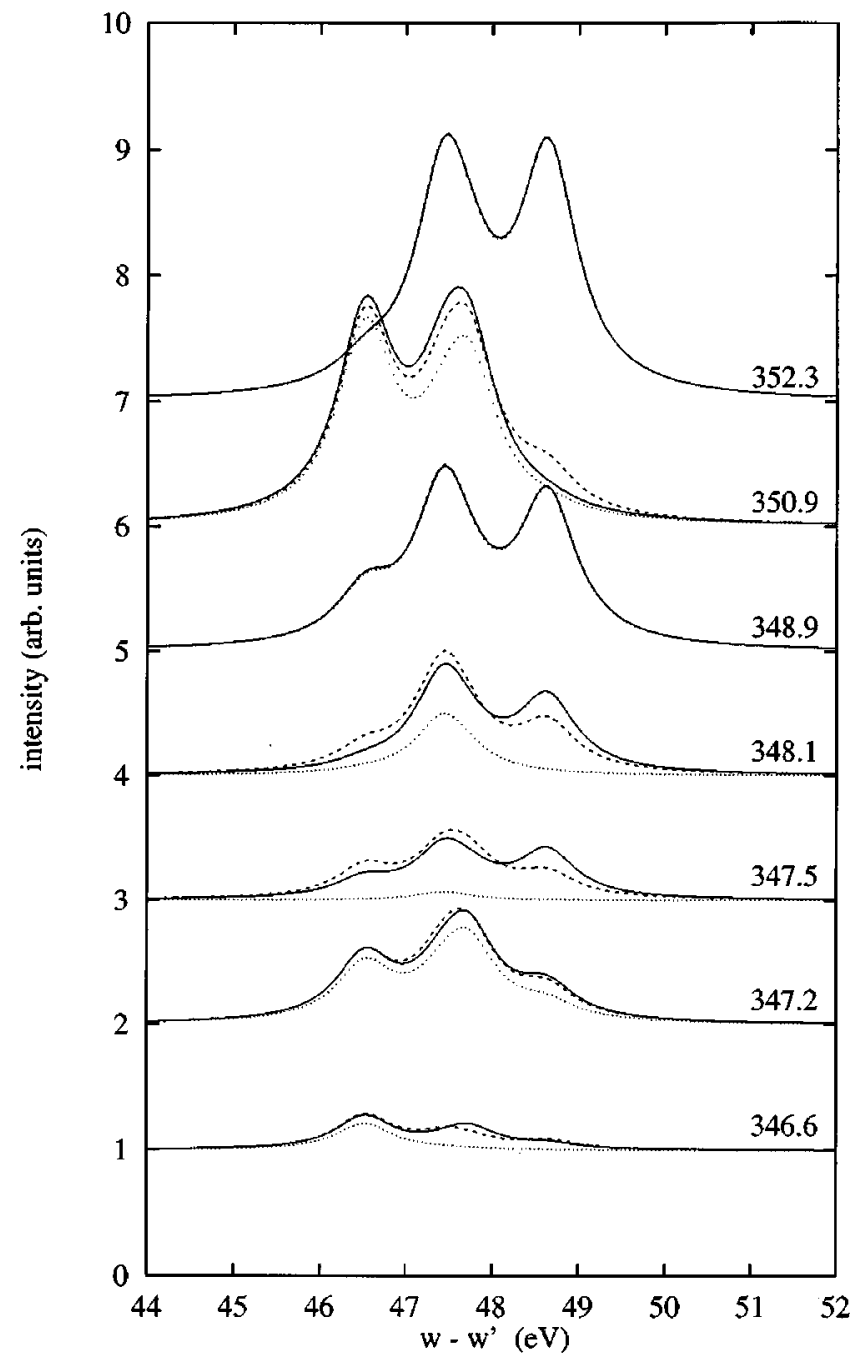

Figure 27. Cross sections through Figure 22 at the excitation energies as indicated on the right. The dotted lines give the decay of only the excited state at precisely the energy indicated. The dashed lines include the Lorentzian tails of the neighboring states, and the solid lines include interference effects. (Reprinted with permission from ref 31. Copyright 1996 American Physical Society.)

a sum rule. Relatively large interference effects can be seen for the spectra excited at 347.5 and $348.1 \mathrm{eV}$. These two energies relate to weak excitations; hence, the decay spectrum is dominated by the Lorentzian tails of the neighbors. That is, interference effects are largest if two channels have a comparable effective strength at a certain energy. The experimental results are reproduced with these calculations.

A topic not discussed in this paper is the consequences of intermediate-state decay/relaxation, also known as postcollision interactions, ${ }^{81}$ as observed in the experimental spectra. These decay channels do give rise to nonresonant X-ray emission. Far above the edge the situation is clear and these nonresonant channels are the normal fluorescence lines at constant energy, but the nonresonant channels close to and even at resonance present a theoretical challenge as they can (and will) be different both from resonant $\mathrm{X}$-ray emission and also from the normal nonresonant channels. To shed light on this situation, one has to understand in great detail what happens exactly in the various relaxation and decay channels, both elastic as inelastic. In solids this is a dramatically complex situation and little is known quantitatively. The situation is a bit better known in atomic spectra, and the interested reader is referred to those papers, for example, the recent review by Gel'mukhanov and Agren. ${ }^{53}$

\section{B. 2p3d Resonant X-ray Emission}

The first high-resolution $2 p 3 d$ resonant X-ray emission experiments have been carried out by the group of Nordgren.17,18,57,86 Figure 28 shows the resonant 2p3d X-ray emission spectra of MnO. The absorption spectrum of $\mathrm{MnO}$ has been discussed in section II. On various positions of the $2 p$ X-ray absorption spectrum, the resonant 2p3d X-ray emission spectrum has been measured as indicated at the top of Figure 28. The spectra have been simulated with an atomic multiplet calculation of the $2 \mathrm{p} 3 \mathrm{~d}$ matrix elements fed into the Kramers-Heisenberg formula. (In the curves in Figure 28, interference effects have been omitted.) It can be seen that the experimental spectra are essentially reproduced. ${ }^{16}$ Including ligand fiel ds, charge transfer, and interference effects improves the comparison. ${ }^{13}$

In Figure 28 one can see the elastic peak related to transitions of the $3 d^{5}$ ground state via any $2 p^{5} 3 d^{6}$ intermediate state back to itself. In addition, one observes peaks at constant energy loss, for example, at about $-4.0 \mathrm{eV}$. These peaks relate to dd transitions. They essentially map the Tanabe-Sugano diagrams of the electronic configurations of a $3 d^{5}$ ground state. It is important to notice that the relative intensity of these various peaks is not constant over the excitation energies but varies. This is an indication of the relative matrix elements from the $2 p^{5} 3 d^{6}$ intermediate states back to the $3 d^{5}$ states. In spectra $f, g$, and $h$, one also observes a peak shifting to higher energy losses. This is a signal of the off-resonant $2 \mathrm{p} 3 \mathrm{~d}$ fluorescence that is visible because of the relaxation of the intermediate states.

\section{Charge-Transfer Transitions and dd (ff) Transitions}

Butorin recently published a series of $2 \mathrm{p} 3 \mathrm{~d}$ resonant X-ray emission spectra experiments for $\mathrm{FeCO}_{3}$, $\mathrm{CoO}, \mathrm{NiO}$, and $\mathrm{LaCOO}_{3} 14$. The 2p3d spectra of $\mathrm{FeCO}_{3}$ 


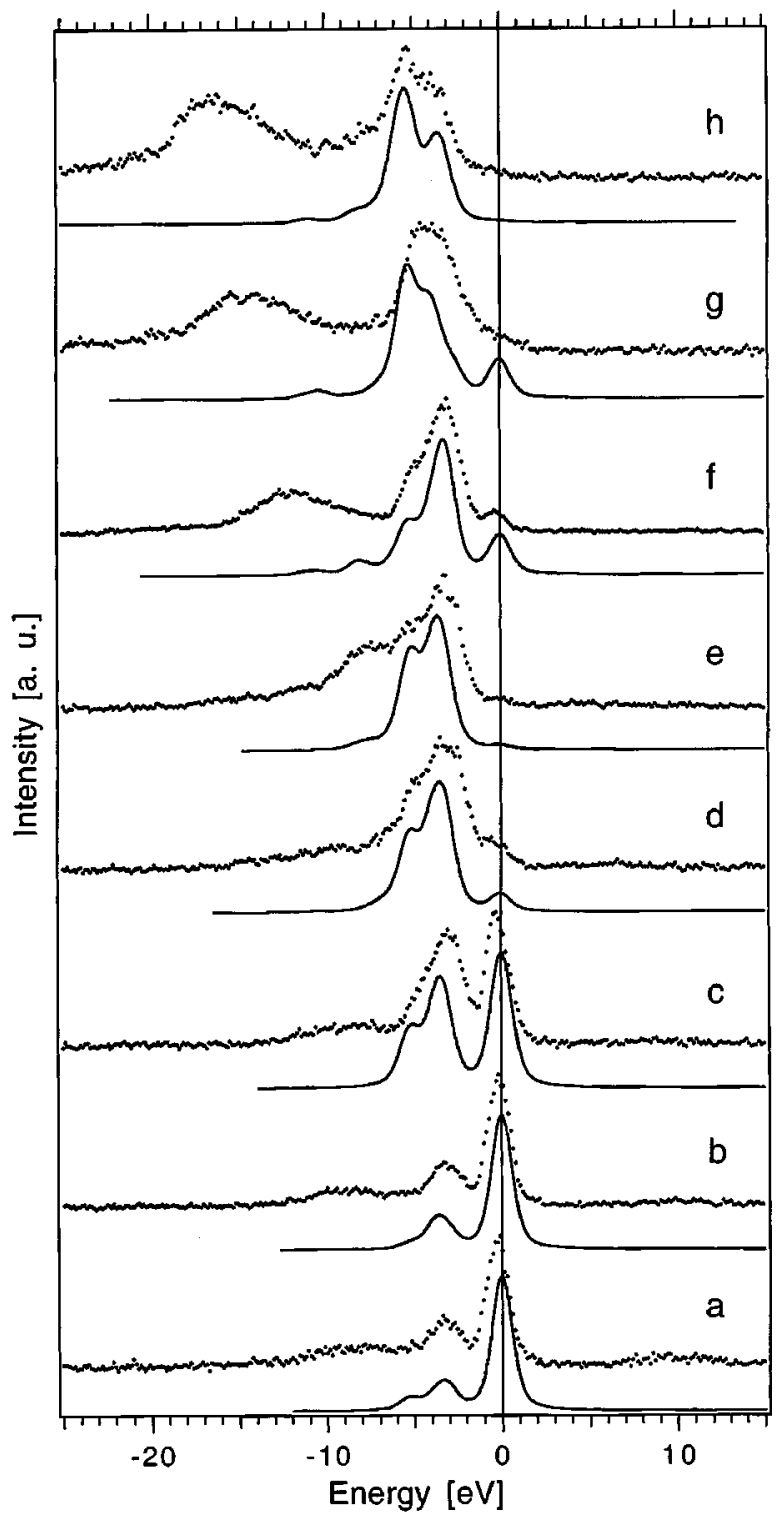

Figure 28. $M n$ 2p3d resonant X-ray emission spectra of $\mathrm{MnO}$ recorded at different excitation energies near the $\mathrm{Mn}$ $2 p$ threshold, as indicated in the X-ray absorption spectrum at the top. The experimental spectra $(\cdots)$ are plotted as energy loss spectra and are compared to atomic multiplet calculations for the $\mathrm{Mn}^{\prime \prime}$ ion (-). (Reprinted with permission from ref 16. Copyright 1996 American Physical Society.)

could be nicely explained with ligand-field multiplet calculations, while the other oxides needed the inclusion of charge-transfer effects. The calculations confirm that it is crucial to include the correct angle between incoming and outgoing X-rays, i.e., the correct combinations of X-ray polarization. The $\mathrm{NiO}$ and $\mathrm{CoO}$ spectra excited at the charge-transfer satellites of the $2 p$ X-ray absorption reveal large satellites in the resonant X-ray emission. These satellites are positioned at a similar X-ray energy as the offresonance $2 \mathrm{p} 3 \mathrm{~d}$ X-ray emission, but the charge transfer multiplet calculations show that they are caused by resonant satellites related to a final state with a ligand hole. The transition can be envisaged as $3 d^{N} \rightarrow 2 p^{5} 3 d^{N+1}+2 p^{5} 3 d^{N+2} L \rightarrow 3 d^{N+1} L$, where the initial and final states are simplified to their main configuration, i.e., one observes the charge-transfer

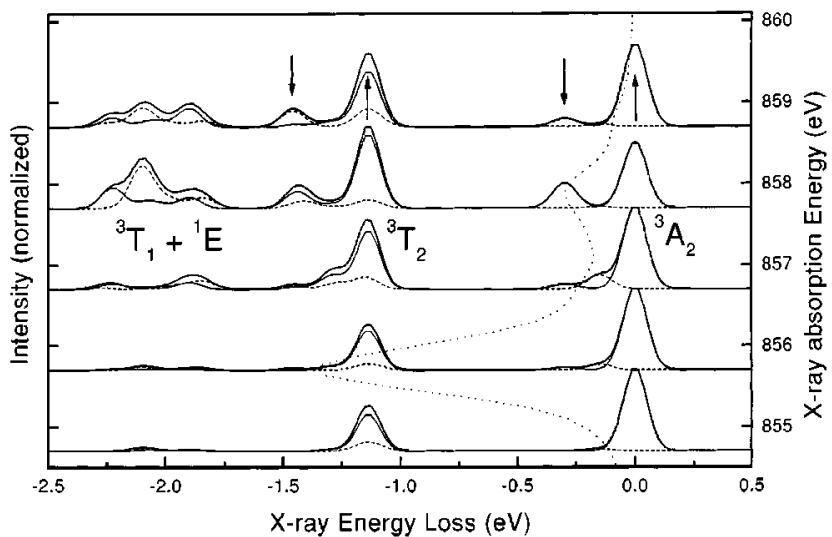

Figure 29. Resonant $2 \mathrm{p} 3 \mathrm{~d} \mathrm{X}$-ray emission spectra of a $\mathrm{Ni}$ system in octahedral symmetry, using the crystal-field parameter of $\mathrm{NiO}$. Indicated are $\mathrm{F}_{\mathrm{xx}}$ scattering $(-), \mathrm{F}_{\mathrm{zx}}$ scattering (- - ), and the total scattering (-). The X-ray absorption spectrum is given with dots. The symmetries of the states are given at the middle spectrum. (Reprinted with permission from ref 41 . Copyright 1998 American Physical Society.)

excitation. The situation of the $3 \mathrm{~d} 4 \mathrm{f}$ resonant X-ray emission spectra of the rare-earth metals is very interesting. One can beautifully observe the resonances of the charge-transfer satellites. ${ }^{15,19,73}$ In addition, one observes ff transitions from which one can determine the crystal-field values of the $4 \mathrm{f}$ electrons. ${ }^{14}$

\section{Coster-Kronig Transitions}

Hague and co-workers ${ }^{59}$ recently showed that in the case of resonant X-ray emission of $\mathrm{TiCl}_{4} \mathrm{~mol}$ ecules, it is not enough to include only the direct resonances as described with eq 18. In addition, the three-step transition via a Coster-Kronig decay channel must be added to describe the observed spectral shapes.

$\mathrm{I}\left(\omega, \omega^{\prime}\right) \sim$

$\left|\sum_{X_{1} X_{2}} \frac{\left\langle 3 d^{0}+3 d^{1} \underline{L}\left|\hat{e}^{\prime} \cdot r\right| X_{2}\right\rangle\left\langle X_{2}\left|H_{C K}\right| X_{1}\right\rangle\left\langle X_{1}|\hat{e} r| 3 d^{0}+3 d^{1} L\right\rangle}{\left(E_{X_{1}}-E_{3 d_{0}}-\hbar \omega-i \Gamma_{2 p}\right)\left(E_{X_{2}}-E_{3 d^{0}}-\hbar \omega-i \Gamma_{2 p}\right)}\right| 2$

Equation 19 shows the Kramers-Heisenberg formula for this three-step pathway. The initial and final states are $3 d^{0}+3 d^{1} L$. The intermediate states $X_{1}$ and $X_{2}$ have a configuration $2 p^{5} 3 d^{1}+2 p^{5} 3 d^{2} L$. In ref 59 , transitions to the continuum have also been included. It was shown that the experimental spectral shapes could only be described correctly with the inclusion of both the Coster-Kronig channel and the explicit inclusion of continuum states.

\section{Spin-Flip Transitions}

Above it was shown that resonant $2 \mathrm{p} 3 \mathrm{~d}$ X-ray emission can give information on dd excitations and charge-transfer excitations. Also, other low-energy excitations can be studied, for example, it has been shown ${ }^{77}$ that spin-flip transitions can be observed in resonant 2p3d and 3p3d X-ray emission of nickel and copper oxides. ${ }^{41}$

Figure 29 shows the resonant 2p3d X-ray emission 
spectra at the $2 p \mathrm{X}$-ray absorption spectrum of $\mathrm{NiO}$. This figure is related to Figure 25 discussed above. The resonant elastic peak of ${ }^{3} \mathrm{~A}_{2}$ symmetry is visible at $0.0 \mathrm{eV}$, and the dd transitions to the ${ }^{3} \mathrm{~T}_{2},{ }^{3} \mathrm{~T}_{1}$, and ${ }^{1} \mathrm{E}$ states are visible at energies of $1.1 \mathrm{eV}$ and at about $2.0 \mathrm{eV}$ energy loss. However, some additional peaks are visible at low-energy loss. F or example, a peak is visible at about $0.25 \mathrm{eV}$. This peak can be assigned to a spin-flip transition in which a $\mathrm{Ni}^{\prime \prime}$ ion flips its spin state from +1.0 to -1.0 (or reversed). The consequence of this spin flip is that the antiferromagnetic neighbors now see an apparent ferromagnetic neighbor and the energy involved is the energy loss observed in resonant X-ray emission. The spinflip peak is highest at an excitation energy of $858 \mathrm{eV}$ that is at the shoulder of the $2 p$ X-ray absorption spectral shape. The reason is that this shoulder relates to a state in which the $2 p$ and $3 d$ electrons are essentially antiparallel aligned, in contrast to the parallel alignment of the main peak. This antiparallel alignment facilitates the spin-flip transitions to occur.

It is good to note that in the literature two completely distinct transitions are called spin flip. There is the spin flip as described above that is related to the transition of a $\mathrm{ms}=-1$ state to a ms $=+1$ state. The other usage of the term spin flip is given to transitions in which the spin 'flips' from, for example, a sextet state to a quartet state. These types of 'spin flip' can be seen in all but the elastic transition in Figure 28. The total spin state has been modified in such a spin-flip transition. In my opinion it is better to refer to such transitions simply as dd transitions.

The measurement of spin-flip transitions is important for the study of the antiferromagnetic coupling in transition-metal oxides. There is, however, an experimental complication which is that the overall resolution of the experiment should preferably be on the order of $0.2 \mathrm{eV}$. This is at the edge of the present technology and still suffers from low intensities. A first measurement has been performed by Kuiper and co-workers using the 3p3d resonance instead of $2 p 3 d .77$ In the future, more studies of the spin-flip effect are expected to yield important new information on the magnetic couplings in transition-metal oxides and other transition-metal systems.

From the discussion of the $2 \mathrm{p} 3 \mathrm{~d}$ resonant inelastic $X$-ray scattering, it is concluded that there are many aspects that must be included for a correct description of the spectral shapes. It depends essentially on the resolution (and system) used and what excitations and pathways must be included. In principle, one can extend the model further by adding other configurations and pathways. I mportant excitations one can observe are charge-transfer transitions, dd transitions, and spin-flip transitions. Excitations into the continuum and Coster-Kronig three-step pathways could until now only be establ ished in molecular systems. This must be related to a difference in electronic structures between molecular systems and solids.

\section{X-ray Magnetic Circular Dichroism}

$X$-ray magnetic circular dichroism (X-MCD) is an important phenomenon in both X-ray absorption and
$X$-ray emission. The magnetic structure of a system is studied by making use of circular or linear polarized X-rays. In the last 10 years many papers have been published on X-MCD. In this review, some general features of $X-M C D$ are discussed. A recent overview is given by Duda. ${ }^{47}$ The focus is on X-ray emission MCD, and in line with the historic development, X-ray absorption MCD is introduced first.

\section{A. MCD in X-ray Absorption}

The MCD effect in X-ray absorption is defined as the difference in absorption between left- and rightpolarized X-rays. A nonzero MCD effect is observed only in magnetic systems (the natural dichroism effects are neglected). The MCD effect has a maximum if the polarization vector of the X-ray is parallel or antiparallel to the magnetization vector of the system.

The MCD effect in X-ray absorption has been measured for the first time by Schütz and coworkers. ${ }^{49}$ They showed that the absorption cross section is different for left- and right-polarized X-rays. The difference between the left- and right-polarized X-rays was shown to be proportional to the spinpolarized density of states. Using the proportionality between X-ray absorption ( $\mathrm{I}_{\mathrm{XAS}}$ ) and the density of state $(\rho)$ as the starting point, one obtains

$$
\mathrm{I}_{\mathrm{MCD}} \equiv \frac{\mathrm{I}_{\mathrm{XAS}}^{\mathrm{L}}-\mathrm{I}_{\mathrm{XAS}}^{\mathrm{R}}}{\mathrm{I}_{\text {XAS }}^{\mathrm{L}}+\mathrm{L}_{\mathrm{XAS}}^{\mathrm{R}}}=\mathrm{P} \frac{\rho_{+}-\rho_{-}}{\rho_{+}+\rho_{-}}
$$

where $\mathrm{I}^{\mathrm{L}}$ denotes left- polarized $\mathrm{X}$-rays and $\mathrm{I}^{\mathrm{R}}$ denotes right-polarized X-rays. $\rho_{+}$and $\rho_{-}$relate to, respectively, spin-up and spin-down density of states. The proportionality factor $\mathrm{P}_{e}$ is the Fano factor. The original paper used the iron $\mathrm{K}$ edge as an example and did find a small effect, in other words, did find a Fano factor of approximately 0.01 . It was later shown that this small MCD effect was particular to the use of a 1s core level and was related to the absence of a core hole spin-orbit coupling. For details on many aspects of this effect, the reader is referred to the book Spin-orbit influenced spectroscopies of magnetic solids. ${ }^{48}$

If a core level has spin-orbit coupling, the MCD effect in X-ray absorption is much larger. If one neglects the overlap of the core and valence wave functions and uses single-electron transition matrix elements, one can simply explain the size of the MCD effect. ${ }^{49} \mathrm{~F}$ or example, $\mathrm{p} \rightarrow \mathrm{d}$ transitions have relative magnitudes for parallel and antiparallel alignments at the $2 p_{3 / 2}$ edge of $P_{3 / 2}=5$ and $A_{3 / 2}=3$, respectively. The related numbers at the $2 p_{1 / 2}$ edge are, respectively, $P_{1 / 2}=1$ and $A_{1 / 2}=3$. This directly yields the relative intensity of the $2 p_{3 / 2}$ and $2 p_{3 / 2}$ edges of $8: 4$. The MCD effect is given as $(P-A) /(P+A)$, leading to $+1 / 4$ for the $L_{3}$ edge and $-1 / 2$ for the $L_{2}$ edge.

An important development in the use of X-MCD was the development of sum rules by Thole and coworkers. ${ }^{107}$ It was shown that the integral of the $X-M C D$ signal could be related to the ground-state expectation value of the orbital moment $\left\langle L_{z}\right\rangle$. A second sum rule was postulated relating the weighted integration of the $L_{3}$ edge plus the $L_{2}$ edge to an effective 
spin moment. Many papers deal with the validity, derivation, and experimental complications of the sum rules. The reader is referred to the Theo Thole memorial issue of the J ournal of Electron Spectroscopy. ${ }^{34}$

Recently, the X-MCD signal at the $L_{2,3}$ edges has been used as a probe signal for microscopy using a photoemission el ectron microscope (PEEM).85,100,102 In addition, the MCD signal has been used for nanosecond time-resolved studies by the group of Alain Fontaine. ${ }^{79}$

\section{MLD in X-ray Absorption}

Magnetic linear dichroism (MLD) is essentially the angular dependence of the absorption cross section. This is an often used technique in X-ray absorption, in particular to study directional variations in the electron density, used, for example, for surfaces and single crystals and to disentangle dipole and quadrupole contributions. ${ }^{101}$ In 1985, Thole and co-workers ${ }^{109}$ predicted a strong magnetic effect on the polarization dependence, an effect that became studied as X-MLD in the last 15 years.

In contrast to $X-M C D$ that probes the magnetic direction, X-MLD only probes the orientation of the magnetic moment, in other words it probes the squared expectation value of the magnetic moment. This automatically implies that X-MLD can intrinsically not make a distinction between magnetic effects and other effects that cause a directional variation of the absorption cross section. To study a magnetic effect with X-MLD, one al ways has to make sure that the effect is indeed magnetic in origin, for example, by a measurement of the same system without being magnetically ordered. The same reasoning applies to MLD in X-ray emission.

\section{B. MCD in X-ray Emission}

In 1991, Strange and co-workers ${ }^{104}$ pointed out that dichroism in X-ray emission spectra can be studied with incident photons that are circularly polarized. It was argued that such an experiment would probe the spin-polarization of the valence band. These experiments are discussed below, where soft X-ray and hard X-ray experiments will be differentiated, i.e., decay to metal $2 p$ and metal $1 s$ core holes, respectively.

If one uses circularly polarized X-rays for the excitation process, naturally this has consequences for the resonant X-ray emission spectra. The basic idea is that the use of circular polarization for the $2 p$ core excitation will create an imbalance between $2 p$ spin-up and spin-down core holes (with respect to the magnetization of the $3 d$ valence electrons). This imbalance implies that detecting the decay of the $3 d$ valence band el ectrons to the polarized $2 p$ core hole will create an imbalance in the spectral shape that is proportional to the spin polarization of the conduction band. With this starting point in mind, many experiments have been carried out.9,22,58,68 It turned out that the situation in the soft X-ray range is complex because of both experimental and theoretical complications.

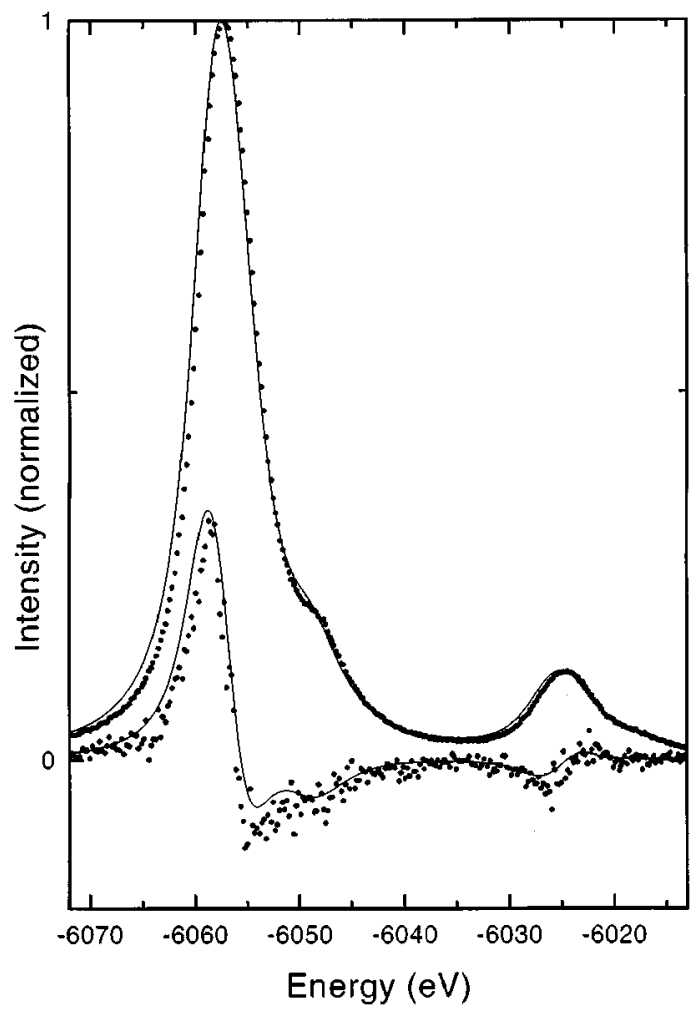

Figure 30. Comparison of the calculated $2 p 3 d X$-ray emission spectral shape $(-)$ with the $2 p_{3 / 2} 3 d$ experimental results $(\cdots)$. (Reprinted with permission from ref 42 . Copyright 1997 American Physical Society.)

On the level of a single-particle analysis, al ready the situation is complex. The single-particle analysis assumes that the MCD effect can be described by the $p \rightarrow d$ matrix elements only. This approach can be found in the works of Erskine and Stern, ${ }^{49}$ Stöhr and $\mathrm{Wu},{ }^{103} \mathrm{~J} \mathrm{o}$ and co-workers, ${ }^{65-67}$ and Kuiper. ${ }^{76}$ In the last reference the argument was used that spin is not a constant of motion in the $2 p$ core hole state. Because of the large core hole spin-orbit coupling, the spin of the core hole will not remain aligned with the spin of the photoelectron. This has direct consequences on the nonresonant and resonant X-ray emission MCD effects. ${ }^{76}$

An important experimental complication is the selfabsorption-caused saturation effects. A high concentration of the element that is absorbing the X-rays implies that an emitted X-ray emission signal can be re-adsorbed before leaving the sample to be measured at the detector. Saturation effects affect the X-ray emission spectral shapes, including the MCD effects. In principle, they can be corrected for, but in the case of concentrated systems, this is a nontrivial exercise. ${ }^{10}$ Braicovich and co-workers recently showed that by using the 2p3s X-ray emission channel, selfabsorption effects can be excluded. ${ }^{11}$ This paper used the fact that there is an angular dependence of the fluorescence and of the MCD effect therein. 42,108

In the hard X-ray range, most of the experimental complications disappear. However, it is known that the $\mathrm{K}$-edge MCD effect is very small. ${ }^{49}$ There has been a series of studies on the MCD effect of the rare earth $L$ edges. Figure 30 shows the MCD effect in the $2 p_{3 / 2} 3 d X$-ray emission spectrum of $\mathrm{Gd}$ metal. $5,74,75$ By taking care of the multiplet interactions and the 

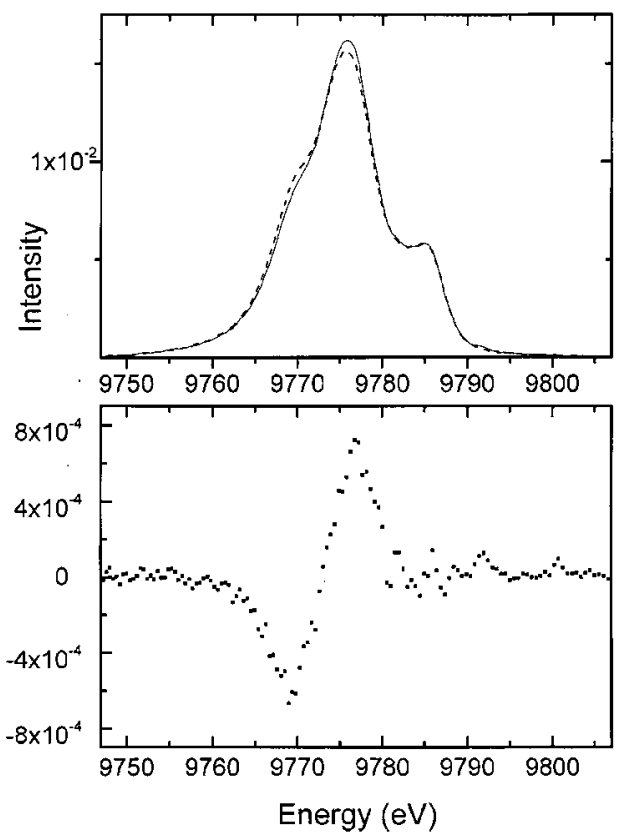

Figure 31. (top) $2 p_{1 / 2} 4 d X$-ray emission spectral shape as a function of X-ray emission energy at detection angles of $30^{\circ}(\cdots)$ and $70^{\circ}(-)$. (bottom) Difference spectrum $\left(70^{\circ}-\right.$ $30^{\circ}$ ). (Reprinted with permission from ref 40 . Copyright 2000 American Physical Society.)

angles between incident X-rays, emitted X-rays, and magnetic field, one can simulate the experimental spectra as shown in Figure 30.

\section{MLD in X-ray Emission}

In analogy with absorption magnetic linear dichroism (MLD), the X-ray emission MLD effect is the angular dependence of the X-ray emission cross section with respect to the magnetization vector.

Figure 31 shows the magnetic linear dichroism effect in the $\mathrm{Yb} 2 \mathrm{p}_{1 / 2} 4 \mathrm{~d} X$-ray emission spectrum of $\mathrm{Yb}_{3} \mathrm{Fe}_{5} \mathrm{O}_{12}{ }^{40} \mathrm{X}$-ray emission spectra were recorded at $110^{\circ}$ and $150^{\circ}$ scattering angles, corresponding to an angle between the magnetic field direction and the emitted X-rays of $70^{\circ}$ and $30^{\circ}$, respectively. The bottom spectrum shows the difference between these two detection angles. From this difference spectrum, the MLD spectrum can be derived by extrapolating the spectra to the situation of, respectively, parallel and perpendicular magnetic fields and detection directions. This MLD effect can nicely be reproduced with multiplet calculations. ${ }^{40}$

It is interesting to look at the difference between this MLD experiment and the MCD X-ray emission experiments. In these MCD experiments, the dichroism is part of the excitation step, while in the emission MLD effects discussed above, the linear dichroism is part of the decay step. Note that both the MCD- and emission MLD-integrated intensities are equal to zero. ${ }^{40}$

In the case of emission MLD, this is evident as the total X-ray emission integrated over an edge must be spherically symmetric, because only core levels are involved in the X-ray emission process itself. This implies a zero integrated intensity over the MLD effect in the 2p4d X-ray emission spectrum. In principle, one could envisage a finite emission MLD effect for the $2 p_{1 / 2}$ edge, which is compensated for at the $2 p_{3 / 2}$ edge, similar to the situation in the MCD of soft X-ray absorption. The $2 p$ spin-orbit coupling, however, is so large that the $2 p_{1 / 2}$ and $2 p_{3 / 2}$ states can be assumed to be pure, implying zero integrated intensity for the emission MLD effect. The same is true for the MCD effect, as the electron is excited from a core state to a continuum state some $100 \mathrm{eV}$ above the edge, where no significant MCD effect is expected in the absorption. While the integrated intensity of the MCD and emission MLD effects is zero, their amplitude is proportional to the $4 \mathrm{f}$ magnetization, because it is the $4 \mathrm{~d} 4 \mathrm{f}$ exchange interaction which gives rise to the appearance of the dichroism signal. It therefore shares this sensitivity with the 3d and $4 d$ absorption MCD and absorption MLD spectra of the rare-earth metals. It can be shown that the size of the emission MLD effect appears as a property that is sensitive to the $4 \mathrm{f}$ magnetic moments of the rare-earth metals. This technique is therefore complementary to absorption MCD spectroscopies at the rare-earth $3 d$ and $4 d$ edges, which have to be performed in the soft $\mathrm{X}$-ray regime. This technique can obviously be extended to $3 d$ transition-metal systems where the $1 s 2 p$ and $1 s 3 p$ X-ray emission channels will have a MLD effect that is, in this case, sensitive to the $3 \mathrm{~d}$ magnetic moment. Magnetic linear dichroism in X-ray emission is likely to become a useful new technique in the study of magnetic materials, since it circumvents the intrinsic complications of soft X-ray spectroscopies such as a highvacuum environment and electron detection. Furthermore, the use of hard X-rays implies that the technique is a bulk-sensitive probe and in situ experiments will be possible.

\section{E. Spin-Polarized 1s2p and 1s3p X-ray Emission}

Spin-polarized 1s2p X-ray emission does not make use of any spin detector or circular-polarized X-rays, but instead uses an internal spin reference, namely, the intermediate state with its 1s core hole.

It has been eluded al ready in section III that $1 s 2 p$ and $1 s 3 p$ X-ray emission spectra are spin-polarized. In all models discussed to interpret these spectra there will always be a small exchange interaction between the $1 \mathrm{~s}$ core hole and the $3 \mathrm{~d}$ electrons. The result is that in the calculations each transition is essentially split into two subpeaks split by the 1s3d exchange. The energies involved are only a few meVs, and as a result there will be no visible effects in the spectral shape. So why is this an interesting and in fact very useful effect?

The reason is that in the final state the configurations are $3 p^{5} 3 d^{N}$ and, as discussed, the $3 p 3 d$ exchange interaction is very large, creating in a first approximation a main peak that is spin-up and a satellite at some $15 \mathrm{eV}$ distance that is spin-down. Things are not as easy as this simple division, and to calculate the actual division into spin-up and spindown, it is very useful to make use of the small 1s3d exchange interaction in the calculations. Because the $1 s 2 p$ and $1 s 3 p$ X-ray emission channels will conserve their spin state, the two 1s intermediate states will essentially map the spin-up and spin-down states, refer to the paper of Wang and co-workers for details. ${ }^{121}$ 

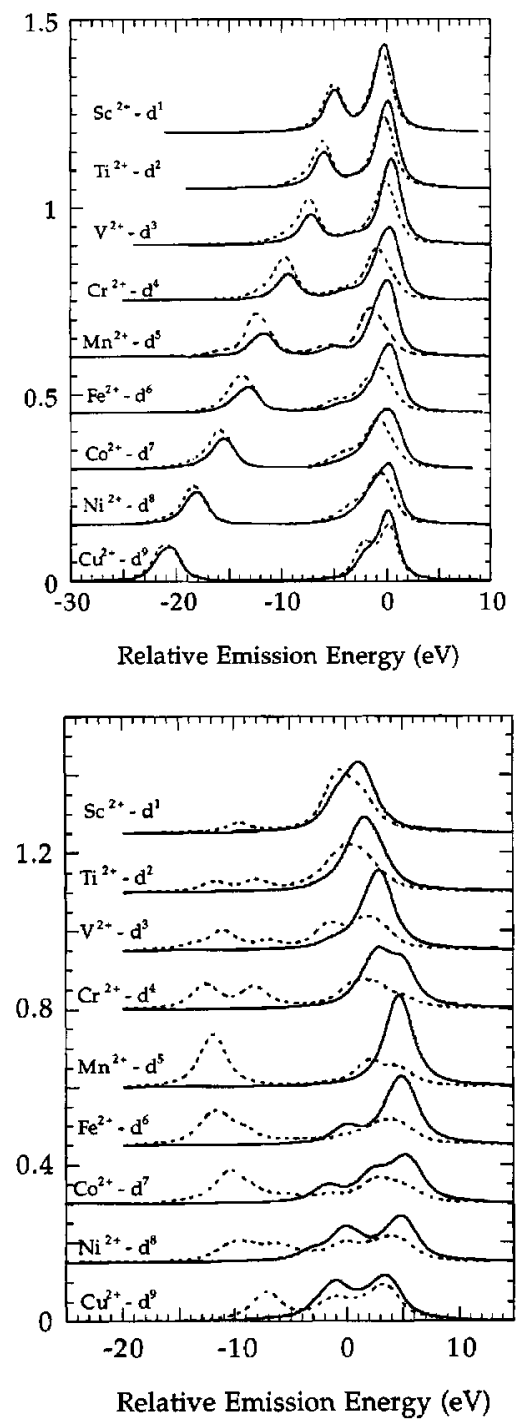

Figure 32. Theoretical spin-polarized 1s2p (top) and 1s3p (bottom) X-ray-emission spectra of the divalent $3 d$ transition-metal ions using the ligand-field multiplet cal culation. Plotted are spin-down $(-)$ and spin-up spectra (..*). (Reprinted with permission from ref 121. Copyright 1997 American Physical Society.)

Figure 32 shows the separation into spin-up and spin-down using ligand-field multiplet calculations. The situation does not change significantly if one includes charge transfer. ${ }^{36,121}$ One can immediately see that the $1 s 2 p$ X-ray emission spectra only have a minor separation into spin-up and spin-down. Both the main peak and the satellite essentially show a shift between spin-up and spin-down. The situation is completely different in the case of the 1s3p X-ray emission spectra. Here a pure spin-down peak is seen for the satellite, while the main peak is mostly spinup. This is particularly clear in the middle of the series where the exchange interaction is largest. It is because of this difference between $1 s 2 p$ and $1 s 3 p$ spectra that the 1s3p spectra can ideally be used to perform spin-selectiveX-ray absorption experiments that will be discussed in section VII.

\section{Selective X-ray Absorption}

Selective X-ray absorption is an experiment that makes use of a particularly chosen X-ray emission decay channel. ${ }^{42}$ One could al so use a photoemission/ Auger channel, but these experiments will not be discussed in this paper. The onset of selective X-ray absorption can be found in the work of Hämäläinen and co-workers, who showed that selective X-ray absorption effectively removes the lifetime broadening ${ }^{61}$ and who later showed the possibility of measuring spin-dependent X-ray absorption. ${ }^{60}$

In section $V$, it was pictorially shown that the lifetime broadening effectively is removed if one measures selective X-ray absorption at resonance. Here the discussion on lifetime broadening disappearance will be extended. Figure 33 shows sche-

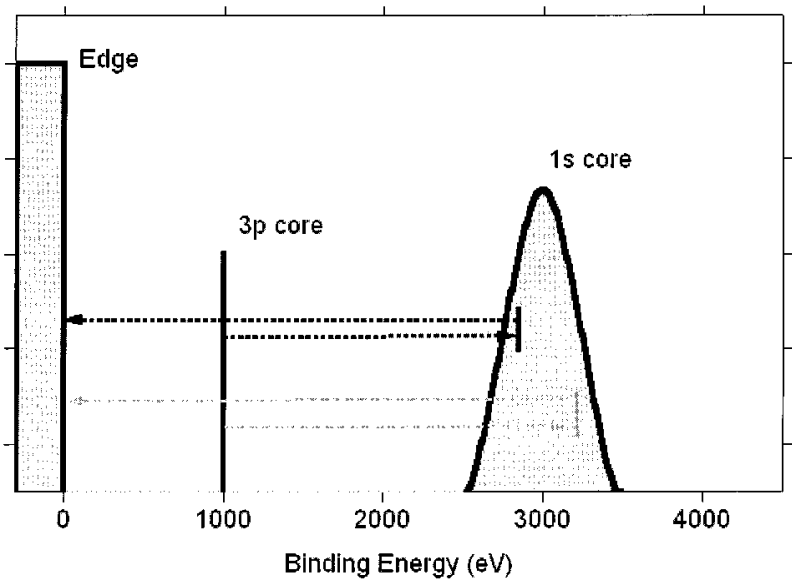

Figure 33. Removal of the lifetime broadening. An el ectron is excited from the broad 1s core level to the edge and a $3 p$ el ectron fills the $1 s$ hole. The detector is set at an energy equal to the binding energy of the $3 p$ state. In that case, it does not matter if the top or bottom transitions do take place (see text).

matically the excitation of a 1s core electron at 3000 $\mathrm{eV}$ to the edge. One detects the $3 p$ electrons that decay to the 1s hole. The lifetime broadening of the 1s level is chosen to be very large, and the 3p level is chosen to be infinitely sharp. Then by putting the energy difference between excitation and decay exactly to $1000 \mathrm{eV}$, one detects only those electrons that go from the $3 p$ level to the 1s level, following the 1s to edge excitation. In this process the broadening of the ls level does not matter, for example, the two processes indicated by the arrows both are possible and are detected at the same energy difference. The exact description of the removal of the lifetime broadening is the Kramers-Heisenberg formula as discussed above. One can rewrite this formula by assuming that there is no interference, which is certainly a correct assumption in the case of 1s core hole intermediate states. This removes the denominator and gives

$$
\mathrm{I}(\omega) \sim \sum_{\mathrm{f}} \sum_{\mathrm{x}}\left\langle\Phi_{\mathrm{f}}|\hat{\mathrm{e}} \mathrm{r}| \Phi_{\mathrm{x}}\right\rangle^{2}\left\langle\Phi_{\mathrm{x}}|\hat{\mathrm{e}} \mathrm{r}| \Phi_{\mathrm{i}}\right\rangle^{2}
$$

The summation of the X-ray emission matrix el ement yields a constant ( XES $_{\text {XE }}$ ) and can be removed from the formula. What remains is analogous to the normal XAFS 


$$
\mathrm{I}(\omega) \sim \sum_{\mathrm{x}}\left\langle\Phi_{\mathrm{x}}|\hat{\mathrm{e}} \mathrm{r}| \Phi_{\mathrm{i}}\right\rangle^{2} \delta_{\mathrm{E}_{\mathrm{f}}-\mathrm{E}_{\mathrm{i}}-\left(\hbar \omega-\hbar \omega^{\prime}\right)}
$$

It can be seen that the matrix element concerns the intermediate-state $\Phi_{x}$, for example, a is state, and the delta function (that is rewritten to a Lorentzian broadening) contains the final state and initial state, without the intermediate state. In other words, the lifetime broadening of the intermediate state is completely removed from the equation. In section $\mathrm{V}$ it was shown that in the case of $2 p$ core holes, interference effects modify this picture a little bit, but the essential conclusion remains that selective X-ray absorption can measure X-ray absorption spectra with a resolution much better than the core hole lifetime broadening.

It was shown that the combination of X-ray absorption and X-ray emission gives us (a) lifetime broadening removed X-ray absorption and (b) spin-, valence, and site-sel ective X-ray absorption.

\section{A. Lifetime Broadening Removed X-ray Absorption}

One can make use of one of these possibilities to improve upon the normal $2 p \mathrm{X}$-ray absorption experiments. That is, one can remove the $2 p$ lifetime broadening of ca. $4 \mathrm{eV}$ and replace it by the $3 \mathrm{~d}$ or $4 \mathrm{~d}$ lifetime broadening of less than $0.5 \mathrm{eV}$. This results in enormously sharper $L_{2,3}$ edges. Figure 34 shows

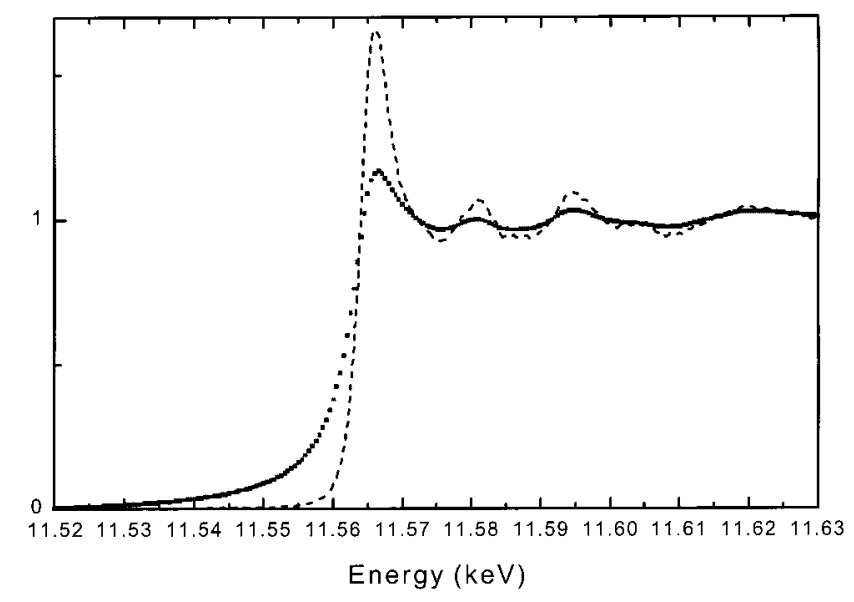

Figure 34. Platinum $\mathrm{L}_{3}$ edge measured with fluorescence yield XAFS $(\cdots)$ and with $2 p_{3 / 2} 3 d$ detected XAFS $(---)$.

the platinum $L_{3}$ edge with fluorescence yield $X$-ray absorption (points) and with $2 p_{3 / 2} 3 d$-detected X-ray absorption (dashed), both measured simultaneously at beamline ID26 at ESRF. Details on the experiment and the analysis will be published elsewhere. As expected, the $L_{3}$ is sharper if the $2 p$ lifetime broadening is removed. This sharpening makes it possible to measure the $L_{3}$ edge with very high resolution, provided the monochromator resolution is very good. The high-resolution XANES spectra can be used to study small variations in the lowest empty states.

\section{B. Valence-, Spin-, and Site-Selective X-ray Absorption}

The central point of selective X-ray absorption is to make use of a particular fluorescence channel to measure the X-ray absorption spectrum. For example, by measuring the X-ray absorption spectral shape at the peak positions of the $\mathrm{K} \beta_{1,3}$ and the $\mathrm{K} \beta^{\prime}$ peaks, one can obtain the spin-selective X-ray absorption spectral shape. ${ }^{43,121}$ This is important for the study of magnetic materials. This applies not only to the XANES region, but just as well to the EXAFS region, which allows for studies on the local geometry that are, for example, separated for their spin. ${ }^{119,120}$

\section{Valence-Selective $X$-ray Absorption}

As shown in Figure 15, the 1s3p fluorescence spectral shapes shift to higher energy with increasing valence. One can tune the energy of the fluorescence detector to the peak position of one valence and vary the energy of the incoming X-ray, thereby measuring the X-ray absorption spectrum of that particular valence. This can be repeated for the other valence. Consequently, one obtains separate X-ray absorption spectra for the various valences present, in other words valence-selective X-ray absorption. ${ }^{56}$

Recently, valence-selective X-ray absorption has been applied to $\mathrm{Fe}^{\prime \prime \prime}{ }_{4}\left(\mathrm{Fe}^{\prime \prime}(\mathrm{CN})_{6}\right)_{3}$ (Prussian Blue). Prussian Blue can be considered the prototype of mixed-valence compounds. It contains two kinds of iron in different oxidation and spin states. The ferrous ion presents a coordination shell constituted by six carbons at $1.92 \AA$ and is surrounded by six nitrogens at $3.04 \AA$ and six ferric ions at $5.08 \AA$. The formal oxidation number is II, and the strong crystal field exhibited by the carbon atoms yields a low-spin ground-state configuration. The ferric ion $\mathrm{Fe}$ "ll has a high-spin configuration. The bulk ratio $\mathrm{Fe}^{\prime \prime \prime}$ to $\mathrm{Fe}^{\mathrm{l}}$ is 4:3. Glatzel and co-workers ${ }^{54}$ showed that the $1 \mathrm{~s} 3 p$ X-ray emission spectrum of Prussian Blue can be simulated by a $4: 3$ ratio of the spectra of $\mathrm{Fe}_{2} \mathrm{O}_{3}$ and $\mathrm{K}_{4} \mathrm{Fe}(\mathrm{CN})_{6}$. Using these two systems as a reference for the Fe $\mathrm{e}^{\prime \prime \prime}$ and $\mathrm{Fe}^{\prime \prime}$ spectra, valence-sel ective X-ray absorption spectra were measured by tuning the crystal analyzer to fluorescence energies that mainly detect contributions from the element in one specific site while the energy of the incident $\mathrm{X}$-ray beam is scanned through the iron $\mathrm{K}$-edge region, including the EXAFS. EXAFS analysis shows good agreement of the $\mathrm{Fe}^{\prime \prime I}$ and $\mathrm{Fe} \mathrm{e}^{\mathrm{Il}}$ sites with X-ray diffraction data. ${ }^{54}$

\section{Spin-Selective $X$-ray Absorption}

Figure 32 in section V.E explained the measurement of spin-polarized X-ray emission spectra. Tuning the detector to a photon energy related to either spin-up or spin-down while scanning through an absorption edge, the spin-polarized X-ray absorption spectrum is measured. From Figure $32 \mathrm{~b}$, it is evident that $\mathrm{Mn}^{\prime \prime}$ is the ideal case to carry out spin-selective X-ray absorption. It turns out that the critical parameter is the number of unpaired $3 d$ electrons. The strong 3p3d exchange interaction will create large splittings that reach a maximum for high-spin $3 d^{5}$. The spin-sel ective X-ray absorption spectrum can be measured for all systems that carry a local moment on a transition-metal ion. This includes ferromagnets, antiferromagnets, and paramagnetic systems.

Spin-selective X-ray absorption was measured for the first time by Hämäläinen and co-workers. ${ }^{60}$ By 
detecting the main peak and the satellite of the $1 s 3 p$ $X$-ray emission spectrum of the antiferromagnet $\mathrm{MnF}_{2}$, they could measure separate X-ray absorption spectra for spin-up and spin-down. It is interesting to compare the spin-sel ective X-ray absorption spectrum with the MCD spectrum of ferromagnets. For ferromagnets, the spin-selective X-ray absorption spectrum identifies with the spin-polarized density of states. As discussed in section IV.A (eq 20), K-edge MCD measures the spin-polarized density of states times the Fano factor. By measuring both the MCD and the spin-sel ective X-ray absorption spectra of the same system, one is able to determine directly the magnitude of the Fano factor, including its potential energy dependence. Measurements on a ferromagnetic MnP sample did show a large energy dependence of the Fano factor, reaching $-4 \%$ at the edge and decreasing to a much smaller value above the edge. ${ }^{43,44}$

The measurement of spin-selective X-ray absorption is easiest for $3 d^{5}$ systems. This, however, does not imply that spin-selective X-ray absorption is impossible for systems that have only one or two unpaired el ectrons. Recently, Wang and co-workers ${ }^{121}$ measured the spin-selective X-ray absorption spectrum of the high-spin $\mathrm{Ni}^{\prime \prime}$ system $\left(\mathrm{PPh}_{4}\right)_{2} \mathrm{Ni}(\mathrm{SePh})_{4}$. Using the spin-polarized signal of the $1 s 2 p$ X-ray emission main peak (cf. Figure 32a), the spin-selective X-ray absorption spectrum was measured.

\section{Site-Selective X-ray Absorption}

A final possibility is site-selective $X$-ray absorption. This possibility arises from the oxygen to metal crossover decay channel, i.e., the $\mathrm{K} \beta$ " peak in Figure 16. Because the oxygen $2 \mathrm{~s}$ peak is present only for the metal atoms that are neighbors to the oxygen, one can measure the X-ray absorption spectra of only those metal atoms. It is important to note that the $X$-ray absorption (including EXAFS) analysis is essentially the same as for normal X-ray absorption and all atoms surrounding the excited atom do contribute to the electron scattering. Only the X-ray excitation and decay process is specific for one type of neighbor, while the electron scattering determining the X-ray absorption spectral shape is not. Site-sel ective X-ray absorption opens a large range of new series of experiments, but the intrinsic low intensity will make it very hard experiments too.

It can be concluded that selective X-ray absorption provides a new useful tool for the study of systems that contain an element in two distinct situations. This adds an extra advantage to the element selectivity of normal X-ray absorption. Because only hard $\mathrm{X}$-rays are involved, further applications are envisaged in in situ characterization in fields such as electrochemistry, heterogeneous catalysis, high-pressure studies, etc.

\section{Conclusions and Outlook}

In this paper a review of high-resolution X-ray emission and X-ray absorption experiments has been given. The emphasis has been on the fundamental aspects of the interpretation and, in addition, on recent advances in this field. The enormous amount of (high-resolution) X-ray emission literature from nonsynchrotron sources has been largely neglected.

In conclusion, it can be remarked that at present there is a situation in which X-ray absorption spectra are analyzed either with density functional theory for the 1s core levels or multiplet theory for all other edges. X-ray emission always involves a core level that necessitates multiplet analysis of the spectral shape. The field of high-resolution X-ray emission is still in a stage of large experimental progress, and more and higher resolution spectra are expected soon. This will in turn cause the theoretical interpretations to become more refined. Some examples of this development are the detection of spin-flip transitions and the realization that Coster-Kronig channels are important for a good description. A picture of X-ray emission will never be complete without the equivalent understanding of the nonradiative decay channels, i.e., resonant photoemission and Auger.

Because high-resolution X-ray emission is a relatively new technique at synchrotron radiation sources, a large part of the experiments carried out today and in the past can be envisioned as prototype experiments. As such, they often belong to the realm of physics. However, a number of dedicated hard X-ray emission beaml ines are projected and/or build at least at the APS, ESRF, and HASYLAB. New soft X-ray emission (resonant inelastic X-ray scattering) beamlines are present at, for example, ALS, MAXLAB, and ELETTRA. These beamlines will make techniques such as RIXS and selective X-ray absorption more accessible to nonexperts, and it is expected that this will largely extend the scope of experiments to be performed. It is hoped that this review serves as a good starting point for the planning of these experiments in fields such as (bio)catalysis, coordination chemistry, electrochemistry, magnetic devices, surface science, etc.

\section{Acknowledgment}

For collaborations and discussions, I thank Steve Cramer, Uwe Bergmann, Pieter Glatzel, Chi-chang Kao, Michael Krisch, Pieter Kuiper, Sergei Butorin, Keijo Hämäläinen, Teijo Åberg, Alain F ontaine, Akio Kotani, and George Sawatzky.

\section{$X$. References}

(1) Åberg, T. At. Phys. High Brilliance Synchrotron Sources, Proc. Workshop 1994, 167-82.

(2) Åberg, T. Phys. Rev. 1967, 156 (1), 35-41.

(3) Arrio, M. A.; Rossano, S.; Brouder, C.; Galoisy, L.; Calas, G. Europhys. Lett. 2000, 51 (4), 454.

(4) Bae, I. T.; Tryk, D. A.; Scherson, D. A. J . Phys. Chem. B 1998 $102,4114$.

(5) Bartolome, F.; Tonnerre, J . M.; Seve, L.; Raoux, D.; Lorenzo, J E.; Chaboy, J .; Garcia, L. M.; Bartolome, J .; Krisch, M.; Rogalev A.; Serimaa, R.; Kao, C.-C.; Cibin, G.; Marcelli, A.J . Appl. Phys. 1998, 83 (11, Pt. 2), 7091-7093.

(6) Bergmann, U.; Glatzel, P.; de Groot, F. M. F.; Cramer, S. P. J Am. Chem. Soc. 1999, 121 (20), 4926-4927.

(7) Bergmann, U.; Grush, M. M.; Horne, C. R.; Demarois, P.; Pennerhahn, J. E.; Yocum, C. F.; Wright, D. W.; Dube, C. E.; Armstrong, W. H.; Christou, G.; Eppley, H. J .; Cramer, S. P. J . Phys. Chem B 1998, 102 (42), 8350-8352. 
(8) Bergmann, U.; Horne, C. R.; Collins, T. J .; Workman, J. M.; Cramer, S. P. Chem. Phys. Lett. 1999, $302(1-2)$, 119-124.

(9) Borgatti, F.; Braicovich, L.; Brookes, N. B.; Ghiringhelli, G.; Tagliaferri, A. J . Electron Spectrosc. Relat. Phenom. 1999, 101103, 467-471.

(10) Braicovich L. Dallera, C. Ghiringhelli, G. Brookes, N. B . Goedkoop, J. B. Phys. Rev. B 1997, 55 (22), 14729-14732.

(11) Braicovich, L.; Van Der Laan, G.; Ghiringhelli, G.; Tagliaferri, A.; Van Veenendaal, M. A.; Brookes, N. B.; Chervinskii, M. M. Dallera, C.; De Michelis, B.; Durr, H. A. Phys. Rev. Lett. 1999, 82 (7), 1566-1569.

(12) Butler, P. H. Point Group Symmetry Applications: Methods and Tables: Plenum Press: New York, 1981.

(13) Butorin, S. M. Private Communication, 1999.

(14) Butorin, S. M. J . Electron Spectrosc. 2000, 110, 213.

(15) Butorin, S. M.; Duda, L. C.; Guo, J . H.; Wassdahl, N.; Nordgren, J .; Nakazawa, M.; Kotani, A. J . Phys.: Condens. Matter 1997, 9 (38), 8155-8160.

(16) Butorin, S. M.; Guo, J .-H.; Magnuson, M.; Kuiper, P.; Nordgren, J. Phys. Rev. B 1996, 54 (7), 4405-4408.

(17) Butorin, S. M.; Guo, J .-H.; Magnuson, M.; Nordgren, J. Phys. Rev. B 1997, 55 (7), 4242-4249.

(18) Butorin, S. M.: Magnuson, M.: Ivanov, K.: Shuh, D. K.: Takahashi, T.; Kunii, S.; Guo, J.-H.; Nordgren, J. J. Electron Spectrosc. Relat. Phenom. 1999, 101-103, 783-786.

(19) Butorin, S. M.; Mancini, D. C.; Guo, J. H.; Wassdahl, N. Nordgren, J .; Nakazawa, M.; Tanaka, S.; Uozumi, T.; Kotani, A.; Ma, Y.; Myano, K. E.; Karlin, B. A.; Shuh, D. K. Phys. Rev. Lett. 1996, 77 (3), 574-577.

(20) Cabaret, D.; Sainctavit, P.; Ildefonse, P.; Flank, A. M. J . Phys. IV 1997, 7 (C2, X.-Ray Absorption Fine Structure, Vol. 1), 157158.

(21) Cabaret, D.; Sainctavit, P.; Ildefonse, P.; Flank, A.-M. Am. Mineral. 1998, 83 (3-4), 300-304.

(22) Caliebe, W. A.; Kao, C.-C.; Berman, L. E.; Hastings, J . B.; Krisch, M. H.; Sette, F.; Hämäläinen, K. J . Appl. Phys. 1996, 79 (8, Pt. 2B), 6509-11.

(23) Caliebe, W. A.; Kao, C.-C.; Hastings, J . B.; Taguchi, M.; Kotani, A.; Uozumi, T.; de Groot, F. M. F. Phys. Rev. B 1998, 58(20), 13452-13458.

(24) Chen, J . G. Surf. Sci. Rep. 1997, 30 (1-3), 5-152.

(25) Cowan, R. D. The Theory of Atomic Structure and Spectra; University of California Press: Berkeley, 1981.

(26) Cramer, S. P.; de Groot, F. M. F.; Ma, Y. J .; Chen, C. T.; Sette, F.; Kipke, C. A.; Eichhorn, D. M.; Chan, M. K.; Armstrong, W. H.; Libby, E.; Christou, G.; Brooker, S.; McKee, V.; Mullins, O. C.; Fuggle, J. C. J. Am. Chem. Soc. 1991, 113, 7937.

(27) de Groot, F. M. F. Ph.D. Thesis, 1991.

(28) de Groot, F. M. F. J . Electron Spectrosc. Relat. Phenom. 1993 $62(1-2), 111-30$.

(29) de Groot, F. M. F. J . Electron Spectrosc. Relat. Phenom 1994, $67(4), 529-622$

(30) de Groot, F. M. F. Physica B 1994, 209, 15

(31) de Groot, F. M. F. Phys. Rev. B 1996, 53 (11), 7099-110.

(32) de Groot, F. M. F. Raman Emiss. X-Ray Scattering, Proc. Workshop 1996, 61-70.

(33) de Groot, F. M. F. Top. Catal. 2000, 10 (3-4), 179-186.

(34) de Groot, F. M. F J . Electron Spectrosc. Relat. Phenom 1997, 86 $(1-3), 5$.

(35) de Groot, F. M. F.; Faber, J .; Michiels, J J . M.; Czyzyk, M. T. Abbate, M.; Fuggle, J. C. Phys. Rev. B 1993, 48 (4), 2074-80.

(36) de Groot, F. M. F.; Fontaine, A.; Kao, C. C.; Krisch, M. H. J. Phys. Condens. Matter 1994, 6 (34), 6875-6884.

(37) de Groot, F. M. F.; Fuggle, J . C.; Thole, B. T.; Sawatzky, G. A Phys. Rev. B 1990, 42 (9), 5459-68.

(38) de Groot, F. M. F.; Fuggle, J . C.; Thole, B. T.; Sawatzky, G. A Phys. Rev. B 1990, 41 (2), 928-37.

(39) de Groot, F. M. F.; Grioni, M.; Fuggle, J. C.; Ghijsen, J .; Sawatzky, G. A.; Petersen, H. Phys. Rev. B 1989, 40 (8), 571523.

(40) de Groot, F. M. F.; Krisch, M. H.; Sette, F.; Vogel, J . Phys. Rev. B 2000, 62 (1), 379-384.

(41) de Groot, F. M. F.; Kuiper, P.; Sawatzky, G. A. Phys. Rev. B 1998, 57 (23), 14584-14587.

(42) de Groot, F. M. F.; Nakazawa, M.; Kotani, A.; Krisch, M. H.; Sette, F. Phys. Rev. B 1997, 56 (12), 7285-7292.

(43) de Groot, F. M. F.; Pizzini, S.; Fontaine, A.; Hämäläinen, K.; Kao, C. C.; Hastings, J . B. Phys. Rev. B 1995, 51 (2), 10451052

(44) de Groot, F. M. F.; Pizzini, S.; Fontaine, A.; Hämäläinen, K.; Kao, C. C.; Hastings, J. B. Physica B 1995, 209 (1-4), 763764.

(45) De Nadai, C.; Demourgues, A.; Grannec, J .; de Groot, F. M. F Phys. Rev. B 2001, 63, 125123.

(46) Dräger, G.; Kirchner, Th.; Bocharov, S.; Kao, C. C. J . Synchrotron Radiat. 2001, 8398.

(47) Duda, L. C. J . Electron Spectrosc. Relat. Phenom 2000, 110 (13), 287-304
(48) Ebert, H.; Schütz, G. E. Spin-Orbit Influenced Spectroscopies of Magnetic Solids; Springer-Verlag: Berlin, 1996.

(49) Erskine, J. L.; Stern, E. A. Phys. Rev. B 1975, 12, 5016.

(50) Finazzi, M.; Brookes, N. B.; de Groot, F. M. F. Phys. Rev. B 1999 59 (15), 9933-9942.

(51) Fontaine, A. Interaction of X-rays With Matter: Absorption Spectroscopy; HERCULES: Grenoble, 1995; Vol. 1

(52) Fuggle, J. C.; Inglesfield. J. E. Unoccupied Electronic States Springer-Verlag: Berlin, 1992.

(53) Gel'mukhanov, F.; Agren, H. Phys. Rep. 1999, 312 (3-6), $87-$ 330.

(54) Glatzel, P.; J acquamet, L.; Bergmann, U.; de Groot, F. M. F.; Cramer, S. P. Angew. Chem., submitted for publication.

(55) Goedkoop, J . B.; Thole, B. T.; Vanderlaan, G.; Sawatzky, G. A.; de Groot, F. M. F.; Fuggle, J . C. Phys. Rev. B 1988, 37, 2086

(56) Grush, M. M.; Christou, G.; Hämäläinen, K.; Cramer, S. P. J. Am. Chem. Soc. 1995, 117 (21), 5895-5896.

(57) Guo, J .-H.; Glans, P.; Skytt, P.; Wassdahl, N.; Nordgren, J .; Luo, Y.; Aagren, H.; Ma, Y.; Warwick, T.; Phys. Rev. B 1995, 52 (15), 10681-4.

(58) Hague, C. F.; Mariot, J .-M.; Guo, G. Y.; Hricovini, K.; Krill, G. Phys. Rev. B 1995, 51 (2), 1370-3.

59) Hague, C. F.; Tronc, M.; Yanagida, Y.; Kotani, A.; Guo, J . H. Sathe, C. Phys. Rev. A 2001, 63, 12511.

(60) Hämäläinen, K.; Kao, C.-C.; Hastings, J . B.; Siddons D. P. Berman L. E. Phys. Rev. B 1992, 46, 14274

(61) Hämäläinen, K.; Siddons, D. P.; Hastings, J . B.; Berman, L. E. Phys. Rev. Lett. 1991, 67, 2850.

(62) Himpsel, F. J .; Karlsson, U. O.; McLean, A. B.; Terminello, L. J .; de Groot, F. M. F .; Abbate, M.; Fuggle, J . C.; Yarmoff, J . A.; Thole, B. T.; Sawatzky, G. A. Phys. Rev. B 1991, 43, 6899.

(63) Hu, Z.; Kaindl, G.; Warda, S. A.; Reinen, D.; De Groot, F. M. F.; Muller, B. G. Chem. Phys. 1998, 232 (1, 2), 63-74.

(64) Hu, Z.; Mazumdar, C.; Kaindl, G.; de Groot, F. M. F.; Warda, S A.; Reinen, D. Chem. Phys. Lett. 1998, $297(3,4), 321-328$.

(65) J o, T. J . Electron Spectrosc. Relat. Phenom 1997, 86 (1-3), 7382.

(66) J o, T.; Parlebas, J . C. J . Phys. Soc. J pn. 1999, 68 (4), 13921397.

(67) J o, T.; Tanaka, A. J . Phys. Soc. J pn. 1998, 67 (4), 1457-1465.

(68) J ournel, L.; Sacchi, M.; Gallet, J .-J .; Hague, C. F.; Mariot, J .M.; Brookes, N. B.; Finazzi, M.; Felsch, W.; Krill, G. J . Electron Spectrosc. Relat. Phenom. 1999, 101-103, 733-738.

(69) Kawai, J. Microbeam Analysis 2000, Proceedings, Williams, D., Shimizu, R., Eds.; Institute of Physics Conference Series: 2000; Vol. 165, p 463

(70) Kawai, J . In Encyclopedia of Analytical Chemistry; Meyers, R. A., Ed.; Wiley: Chichester, 2000; pp 13288-13315.

(71) Kawai, J .; Hayashi, K. J . Electron Spectrosc. Relat. Phenom 1998, 92 (1-3), 243-245.

(72) X-ray Absorption; Koningsberger, D. C., Prins, R., Eds.; Wiley: New York, 1987.

(73) Kotani, A. J . Phys. IV 1997, 7 (C2), 1-8.

(74) Krisch, M. H.; Kao, C. C.; Sette, F.; Caliebe, W. A.; Hämäläinen, K.; Hastings, J . B. Phys. Rev. Lett. 1995, 74 (24), 4931-4.

(75) Krisch, M. H.; Sette, F.; Masciovecchio, C.; Verbeni, R.J . Electron Spectrosc. Relat. Phenom. 1997, 86 (1-3), 159-164.

(76) Kuiper, P. J . Phys. Soc. J pn. 2000, 69 (3), 874-877.

(77) Kuiper, P.; Guo, J .-H.; Sathe, C.; Duda, L.-C.; Nordgren, J .; Pothuizen, J J . M.; de Groot, F. M. F.; Sawatzky, G. A. Phys. Rev. Lett. 1998, 80 (23), 5204-5207.

(78) Lyttle, F. W.; Greegor, R. B.; Panson, A. J . Phys. Rev. B 1988, 37, 1550.

(79) M.Bonfim, M.; Ghiringhelli, G.; Montaigne, F.; Pizzini, S. Brookes, N. B.; Petro, F.; Vogel, J .; Camarero, J .; Fontaine, A. Phys. Rev. Lett. 2001, 86, 3646.

(80) Mahadevan, P.; Sarma, D. D. Phys. Rev. B 2000, 61 (11), $7402-$ 7408

(81) Mori, A.; Kayanuma, Y.; Kotani, A. Prog. Theor. Phys. Suppl 1991, 106, 75-93.

(82) Muller J. E.; J epsen O.; Andersen O. K.; Wilkins J. W. Phys. Rev. Lett. 1978, 40, 720

(83) Natoli, C. R. EXAFS and Near EdgeStructure; Springer: Berlin, 1983.

(84) Natoli, C. R.; Benfatto, M.; Doniach, S. Phys. Rev. B 1986, 34 4682 .

(85) Nolting, F.; Scholl, A.; Stohr, J .; Seo, J . W.; Fompeyrine, J .; Siegwart, H.; Locquet, J. P.; Anders, S.; Luning, J .; Fullerton, E. E.; Toney, M. F.; Scheinfein, M. R.; Padmore, H. A. Nature 2000, 405 (6788), 767-769.

(86) N ordgren, E. J. Roentgen Centen. 1997, 364-380.

(87) Okada, K.; Kotani, A. J. Phys. Soc. J pn. 1992, 61 (2), 449-453.

(88) Okada, K.; Kotani, A. J . Phys. Soc. J pn. 1992, 61 (12), 46194637.

(89) Okada, K.; Kotani, A.; Thole, B. T J . Electron Spectrosc. Relat. Phenom 1992, 58 (4), 325-343.

(90) Peng, G.; de Groot, F. M. F.; Hämäläinen, K.; Moore, J . A.; Wang X.; Grush, M. M.; Hastings, J . B.; Siddons, D. P.; Armstrong, 
W. H.; Mullins, O. C.; Cramer, S. P. J . Am. Chem. Soc. 1994, 116 (7), 2914-2920.

(91) Qian, Q.; Tyson, T. A.; Kao, C. C.; Croft, M.; Cheong, S. W.; Greenblatt, M. Phys. Rev. B 2000, 62 (20), 13472-13481.

(92) Rehr, J. J .; Albers, R. C. Rev. Mod. Phys. 2000, 72 (3), 621654.

(93) Rehr, J. J .; Ankudinov, A.; Zabinsky, S. I. Catal. Today 1998, $39(4), 263-269$.

(94) Robblee, J . H.; Messinger, J .; Fernandez, C.; Cinco, R. M.; Visser, H.; Mcfarlane, K. L.; Bergmann, U.; Glatzel, P.; Cramer, S. P. Sauer, K.; Klein, M. P. J . Inorg. Biochem. 1999, 74 (1-4), 278278.

(95) Rubensson, J . E.; Eisebitt, S.; Nicodemus, M.; Boske, T.; Eberhardt, W. Phys. Rev. B 1994, 50 (13), 9035-9045.

(96) Rubensson, J .; Eisebitt, S.; Nicodemus, M.; Boske, T.; Eberhardt, W. Phys. Rev. B 1994, 49 (2), 1507-1510.

(97) Rueff, J. P.; Kao, C. C.; Struzhkin, V. V.; Badro, I ; Shu, J .; Hemley, R. J .; Hao, H. K. Phys. Rev. Lett. 1999, 82 (16), 32843287.

(98) Rueff, J. P.; Krisch, M.; Cai, Y. Q.; Kaprolat, A.; Hanfland, M.; Lorenzen, M.; Masciovecchio, C.; Verbeni, R.; Sette, F. Phys. Rev. B 1999, 60 (21), 14510-14512.

(99) Sainctavit, P.; Petiau, J .; Benfatto, M.; Natoli, C. R. Physica B (Amsterdam) 1989, $158(1-3), 347-50$

(100) Scholl, A.; Stohr, J .; Luning, J .; Seo, J . W.; Fompeyrine, J .; Siegwart, H.; Locquet, J . P.; Nolting, F.; Anders, S.; Fullerton, E. E.; Scheinfein, M. R.; Padmore, H. A. Ścience 2000, 287 (5455), 1014-1016.

(101) Stöhr, J . NEXAFS Spectroscopy; Springer-Verlag: Berlin, 1992.

(102) Stohr, J .; Scholl, A.; Regan, T. J .; Anders, S.; Luning, J .; Scheinfein, M. R.; Padmore, H. A.; White, R. L. Phys. Rev. Lett. 1999, 83 (9), 1862-1865.

(103) Stöhr, J.; Wu, Y. In New Directions in Research With ThirdGeneration Soft X-ray Synchrotron Radiation Sources; Schlachter, A. S., Wuilleumier, F. J ., Eds.; Kluwer: Dordrecht, 1994.

(104) Strange, P.; Durham, P. J .; Gyorffy, B. L. Phys. Rev. Lett. 1991, 67 (25), 3590-3593.

(105) Sugano, S.; Tanabe, Y.; Kamimura, H. Multiplets of Transition Metal I ons; Academic Press: New-Yiork, 1970.

(106) Tanaka, S.; Kotani, A. J . Phys. Soc. J pn. 1993, 62 (2), 464-467.
(107) Thole, B. T.; Carra, P.; Sette, F.; Vanderlaan, G. Phys. Rev. Lett. 1992, 68 (12), 1943-1946.

(108) Thole, B. T.; Durr, H. A.; Vanderlaan, G. Phys. Rev. Lett. 1995 74 (12), 2371-2374.

(109) Thole, B. T.; van der Laan, G.; Sawatzky, G. A. Phys. Rev. Lett 1985, 55, 2086

(110) Thole, B. T.; Vanderlaan, G.; Butler, P. H. Chem. Phys. Lett. 1988, 14, 9295.

(111) Tsutsumi, K. J . Phys. Soc. J pn. 1959, 14, 1696.

(112) Tsutsumi, K.; Nakamori, H.; Ichikawa, K. Phys. Rev. B 1976, 13, 929.

(113) Tulkki, J .; Åberg, T. Res. Rep.-Helsinki Univ. Technol., Lab. Phys. 1978, 3, 2 pp.

(114) Tyson, T. A.; Qian, Q.; Kao, C. C.; Rueff, J . P.; De Groot, F. M. F.; Croft, M.; Cheong, S. W.; Greenblatt, M.; Subramanian, M. A. Phys. Rev. B 1999, 60 (7), 4665-4674.

(115) van der Laan, G.; Zaanen, J .; Sawatzky, G. A.; Karnatak, R. Esteva, J. M. Phys. Rev. B 1986, 33, 4253.

(116) Van Elp, J.; Tanaka, A. Phys. Rev. B: Condens. Matter 1999, 60 (8), 5331-5339.

(117) von Barth, U.; Grossmann, G. Phys. Rev. B 1982, 25, 5150.

(118) Vvdensky, D. D. I n Unoccupied Electronic States; Fuggle, J . C., Inglesfield, J. E., Eds.; Springer-Verlag: Berlin, 1992.

(119) Wang, X.; Cramer, S. P. J . Phys. IV 1997, 7 (C2), 361-363.

(120) Wang, X.; Randall, C. R.; Peng, G.; Cramer, S. P. Chem. Phys. Lett. 1995, 243 (5-6), 469-473.

(121) Wang, X.; de Groot, F. M. F.; Cramer, S. P. Phys. Rev. B 1997 $56(8), 4553-4564$

(122) Waychunas, G. A. Am. Mineral. 1987, 7289.

(123) Weissbluth, M. Atoms and Molecules; Plenum Press: New York, 1978

(124) Westre, T. E.; Kennepohl, P.; Dewitt, J. G.; Hedman, B.; Hodgson, K. O.; Solomon, E. I. J . Am. Chem. Soc. 1997, 119 (27), 6297-6314.

(125) Zaanen, J .; Sawatzky, G. A.; Allen, J . W. Phys. Rev. B 1985, 55 418.

(126) Zeller, R. In Unoccupied Eectronic States; Fuggle, J . C., Inglesfield, J . E., Eds.; Springer-Verlag: Berlin, 1992.

\section{CR9900681}

